\title{
Physics-Based Online Assessment of Surface Roughness Severity for High-Pressure Turbines
}

\author{
by \\ Zelig (Zhi) Li \\ B. Eng.
}

A thesis submitted to the Faculty of Graduate and Postdoctoral Affairs in partial fulfillment of the requirements for the degree of

Master of Applied Science
in

Mechanical Engineering

Carleton University

Ottawa, Ontario

(C) 2018, Zelig (Zhi) Li 


\section{Abstract}

Surface roughness is a critical indicator of the health of turbine blades, due to its implications on blade surface heat transfer and structural integrity. The present work proposes a physics-based online assessment framework for industrial gas turbine engines (GTE), in order to assess the blade surface roughness in a high-pressure turbine without engine shutdown. The framework consolidates gas path analysis (GPA) based performance monitoring models and meanline turbomachinery analysis using a novel GPA-meanline matching process. This extracts meaningful performance deviation trends from GPA, while resolving the uncertainties associated with the measurements and modeling. To relate efficiency loss to surface roughness severity, a meanline-based system-identification process has been developed to establish the meanline representation of the turbine stage and to incorporate an empirical surface roughness loss correlation system. The roughness loss correlations have been evaluated against recent transonic test data in the literature. A modification to the compressibility correction factor has been made according to the evaluation outcome, which yielded improved loss predictions compared to the experimental measurements.

The successful application of the framework on three-year operational data of a cogenerative GTE validated the framework's potential as an effective online surface roughness monitoring tool. The estimated surface roughness index achieved magnitudelevel and trend agreement with the measurements reported in the literature. 
"No, in all these things we are more than conquerors through him who loved us." - Romans 8:37, ESV 


\section{Acknowledgements}

I wish to thank my supervisor, Professor Jie Liu, for providing me the opportunity to work on this research project. This work could not have been accomplished without his encouragement and sound advice. I would also like to gratefully acknowledge my supervisor from my fourth-year capstone project, Professor Steen A. Sjolander, for introducing me to the field of turbomachinery. I am also truly grateful for his intermittent, but invaluable, guidance and encouragement over the course of this work.

This work could not have been completed without the gracious support from Dr. Houman Hanachi, who was always available for discussion when I encountered an obstacle. I would also like to thank Dr. Avisekh Banerjee, Dr. Feng Gao and other colleagues from the industrial partner, Life Prediction Technologies Inc., for their support of this work. I also greatly appreciate the help from my fellow colleagues in the Carleton community, especially Kun Zhuang, Dr. Ryan Guo, Dr. Ying Chen, Nick Tepylo, Turner Strang, Scott Seymour and Dustin Jee, who were always available to discuss the challenges I was facing. The rigorous editing job performed by Kattie Smilovsky must also be acknowledged.

Above all, I thank God for his faithful and gracious provision for my life. I am tremendously grateful for my family and friends for their unconditional, unwavering and loving support throughout this endeavour. I must also acknowledge the Self-Authoring Program developed by Professor Jordan B. Peterson, which helped me to clean my room, get my act together and navigate through the chaos encountered during this journey of selfdiscovery. 


\section{Table of Contents}

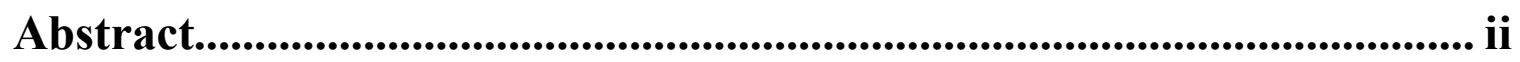

Acknowledgements ......................................................................................... iv

List of Tables .............................................................................................. viii

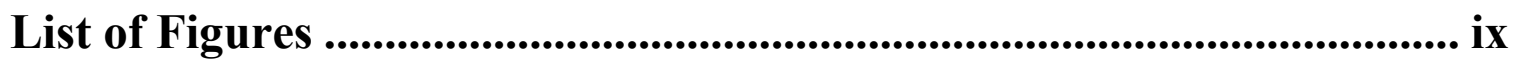

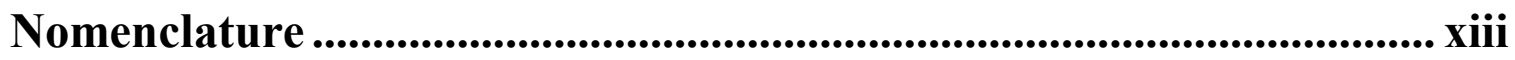

Chapter 1: Introduction ...................................................................................1

1.1 Background and Motivation ........................................................................... 1

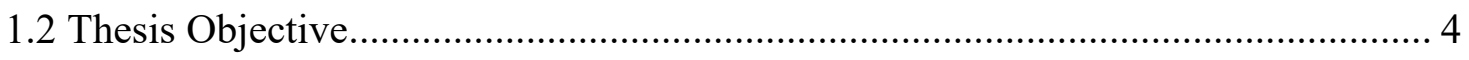

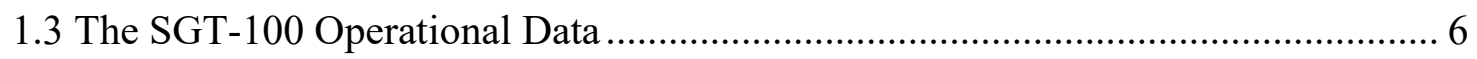

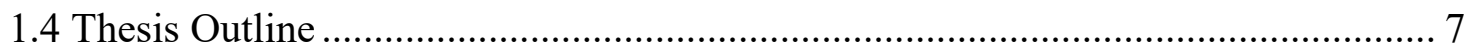

\section{Chapter 2: GTE System and Component Modelling ................................8}

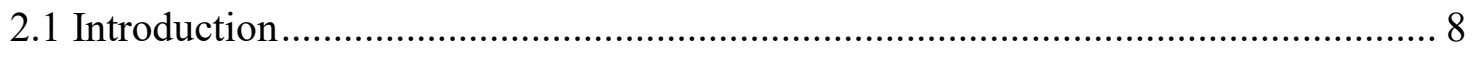

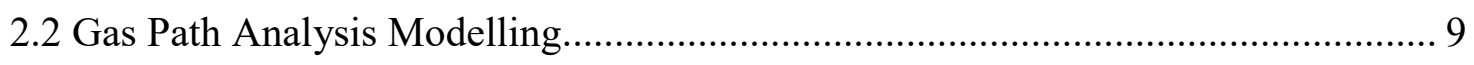

2.2.1 Thermodynamic Modelling for a Single Shaft GTE ................................................... 9

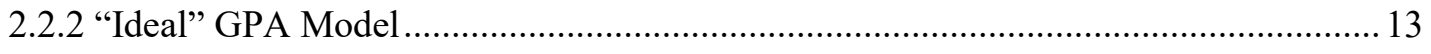

2.2.3 "Turbine-degrading" GPA model ............................................................................ 14

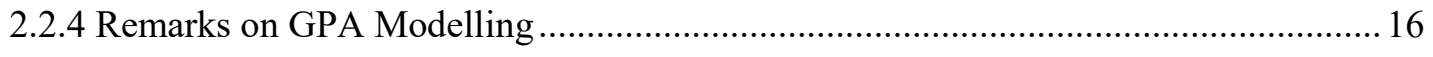

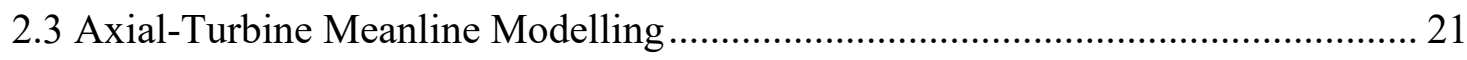

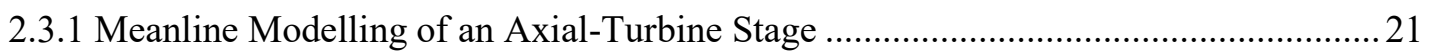




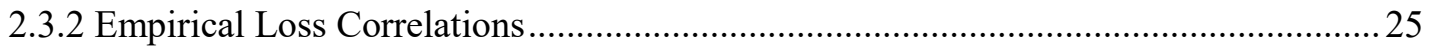

2.3.3 Remarks on Meanline Analysis of Axial Turbines ...................................................... 34

2.4 Loss Correlations for Axial Turbine Blade Roughness Damage........................... 36

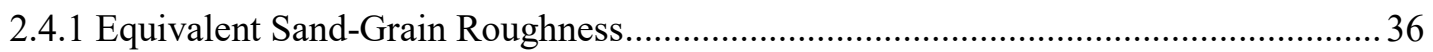

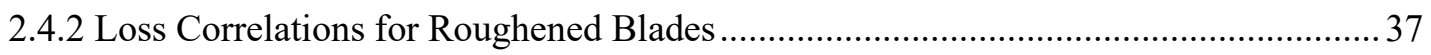

\section{Chapter 3: Validation of the Prediction Method for Blade Roughness Damage Loss under Transonic Flow Conditions......................................41}

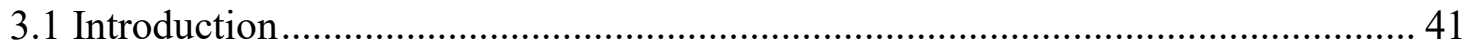

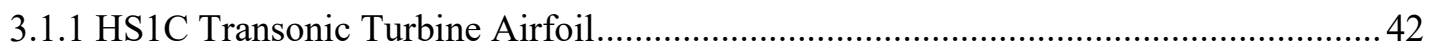

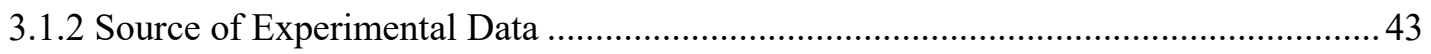

3.2 AMDCKO Profile Loss Prediction for HS1C Cascade ...................................... 46

3.3 Validation of the Roughness Loss Correlations with Compressibility Effects....... 49

3.3.1 Validation of the $\mathrm{K}_{\mathrm{M}}$ Compressibility Correction Factor ........................................ 49

3.3.2 Loss Predictions for HS1C Cascade with Various Roughness Configurations ............53

3.4 Concluding Remarks on the Validation Study............................................. 58

\section{Chapter 4: Online Assessment Framework for the Surface} Roughness of a High-Pressure Turbine.........................................................59

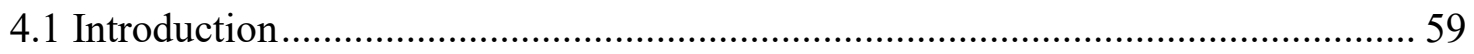

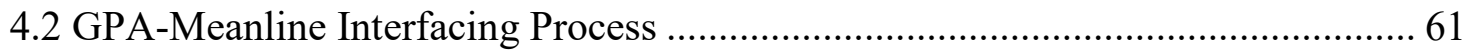

4.2.1 Deviation of Performance Characteristics due to Roughness Fault ............................. 61

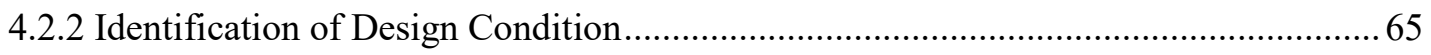

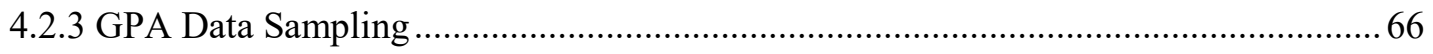

4.2.4 Concluding Remarks on the Interfacing Process ............................................... 71 
4.3 Meanline Roughness Identification Process ..................................................... 73

4.3.1 A Proof-of-Concept Index: The Quarter Surface Coverage Index............................. 76

Chapter 5: Framework Implementation and Discussion .....................78

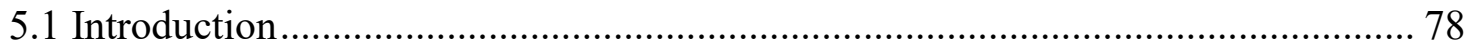

5.2 Ruston/Siemens SGT-100 Typhoon GTE ................................................... 78

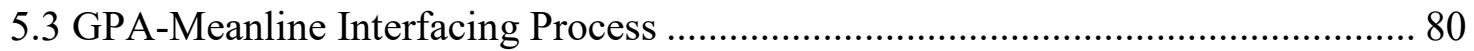

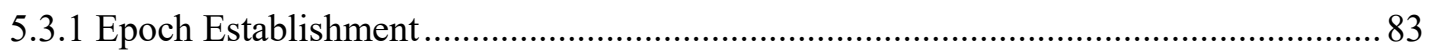

5.3.2 Establishment of the "Actual-Turbine" Performance Characteristics............................ 86

5.4 Meanline Roughness Identification for the HP Turbine .................................... 90

Chapter 6: Conclusions and Recommendations ......................................94

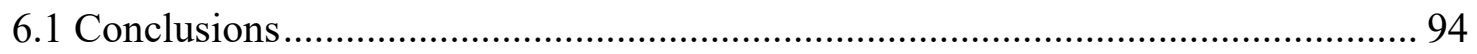

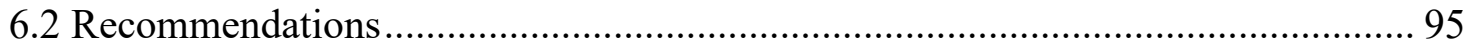

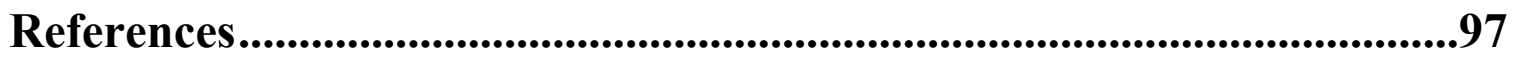

Appendix A: HS1C Cascade Performance Data by Corriveau and Yuan 


\section{List of Tables}

Table 3-1: Summary of blade geometry and the design performance parameters of the HS1C cascade, adapted from Ref. [43] ................................................ 42

Table 3-2: Uncertainties of data measurement and reduction, adapted from Ref. [42]... 43

Table 5-1: Basic performance of the original Ruston Typhoon GTE............................ 79

Table 5-2: Nominal performance for the HP turbine of the Typhoon GTE................... 79

Table 5-3: Epoch placement and corresponding time in the operational history. ............ 83

Table 5-4: Assessed total pressure loss coefficients for various loss components.......... 91

Table 5-5: Comparison of performance parameters between the established meanline model and the reported reference values from Hannis et al. [52]................. 91 


\section{List of Figures}

Figure 1-1: Roughened surface of the first stage vane of a utility GTE after 8000 hours of service, adapted from Ref. [4] 2

Figure 1-2: Scope of the research objectives with respect to performance-based GTE health monitoring. 4

Figure 1-3: Available ambient and gas path measurements, adapted from Ref. [11]....... 6

Figure 2-1: Three years of GPA health monitoring of operational data from the Typhoon GTE: (a) the $I_{P D}$ index by the ideal model, and (b) the isentropic efficiency decrease and mass flow increase of the turbine subsystem assessed by the turbine-degrading model. 16

Figure 2-2: Gas path parameters estimated by both the ideal and the turbine-degrading GPA model. 19

Figure 2-3: Station numbering and illustrations of velocity triangles for a single turbine stage, adapted from Ref. [16].

Figure 2-4: The expansion process across a turbine stage illustrated on a T-s diagram, adapted from Ref. [17] 23

Figure 2-5: Definition of a turbine airfoil, adapted from Ref. [25]. 28

Figure 2-6: The Ainley \& Mathieson profile loss coefficient data for: a. nozzle blades, and b. impulse blades with a maximum thickness to chord ratio $\left(t_{\max } / C\right)$ of 0.2 , adapted from Ref. [16] and [19] 
Figure 2-7: Trailing edge energy loss coefficients for the nozzle and impulse blades, adapted from Ref. [19] 31

Figure 3-1: Summary of test cases and the nomenclature of roughness patch location from Yuan's study, adapted from Ref. [41]. 45

Figure 3-2: Comparison of the profile loss predictions by both AMDCKO and Zhu \& Sjolander correlations alongside the cascade tunnel measurements for the HS1C transonic turbine cascade. 46

Figure 3-3: Comparison of the $\mathrm{K}_{\mathrm{M}}$ obtained using the experimental data of Yuan with the correlation by Jouini et al...... 50

Figure 3-4: Comparison of the $\mathrm{K}_{M}$ obtained using the experimental data of Yuan with the modified $\mathrm{K}_{\mathrm{M}}$ correlation 52

Figure 3-5: Effect of the roughness starting position (x/c): (a) variation of loss with $\mathrm{x} / \mathrm{c}$, adapted from Ref. [12] and (b) tentative correlation for $K_{x}$ used in the present investigation. 53

Figure 3-6: Total pressure loss coefficient with the medium roughness band $(\mathrm{x} / \mathrm{c}=0.25$, $\Delta \mathrm{s} / \mathrm{c}=0.475$ ): (a) total pressure loss coefficient and (b) incremental loss by roughness. 55

Figure 3-7: Total pressure loss coefficient with the narrow roughness band $(\mathrm{x} / \mathrm{c}=0.25, \Delta \mathrm{s} / \mathrm{c}$ $=0.25$ ): (a) total pressure loss coefficient and (b) incremental loss by roughness. 56

Figure 4-1: Schematic of the proposed online assessment framework for HP turbine surface roughness. 60 
Figure 4-2: Transformation of turbine performance characteristic curves associated with the same $\Omega$ due to different faults, reproduced based on Ref. [47] 62

Figure 4-3: Flow chart for the GPA-meanline interfacing process. 63

Figure 4-4: (a) Reported fouling effect on compressor efficiency of a typical heavy duty GTE, reproduced from Ref. [49], (b) expected trend of $T I T_{D E V}$ corresponding to the compressor fouling effect.. 70

Figure 4-5: Efficiency curves corresponding to same $\Omega_{\text {on }}^{\prime}$ from different epochs reveal the temporal trajectory of the curve transformation due to blade roughening..... 72

Figure 4-6: Flow chart of the proposed meanline surface roughness fault matching process.

Figure 5-1: Flow path geometry of the HP turbine stage, adapted from [52]................ 80

Figure 5-2: Distribution of key performance parameters with various TIT ranges.......... 82

Figure 5-3: Epoch placement and data availability: variation of $T I T^{\prime}$ and placement of epoch for (a) global TIT range, (b) near $T I T_{o n}^{\prime}$, and (c) number of data points corresponding to $T I T_{o n}^{\prime}$ for each $\varepsilon_{i}$ 84

Figure 5-4: The $T T_{D E V}$ variation indicated the effect on $T I T^{\prime \prime}$ by both the short-term fouling and the long-term turbine degradation. 86

Figure 5-5: Constructed turbine performance characteristics based on the results of the turbine-degrading GPA model: (a) downward efficiency curve shift and (b) slightly upward shift of the corrected mass flow....

Figure 5-6: Formulation of the $\Delta \eta_{t}$ trend: (a) the frequency distribution of $\Delta \eta_{t}$ for each established epoch and (b) the final $\Delta \eta_{t}$ deviation trend with operating time. 88 
Figure 5-7: Comparison of the $\zeta_{\eta}$ trend obtained by the present interfacing process using the method of Hanachi et al. [55].

Figure 5-8: Established meanline velocity triangles compared with the streamline curvature analysis outcomes by Hannis et al. [52] for the design condition................. 90

Figure 5-9: Variation of the "Quarter Surface Coverage Index" with the isentropic efficiency loss. 92

Figure 5-10: Growth trend of surface roughness in terms of the "Quarter Surface Coverage Index": (a) comparison with the measurements on the IN939 blade samples from Ruston HP turbines, (b) comparison with all available measurements by Tarada [7]. 


\section{Nomenclature}

\section{$\underline{\text { Symbol }}$}

A

AR

C

$C_{x}$

CFM

$C p$

$D P$

EGT

$f$

$h_{0}$

$h$ or $\mathrm{H}$

$i$

$I_{P D}$

$K_{I}$

$K_{\text {mod }}$

$K_{L}$

\section{$\underline{\text { Description }}$}

Annulus gas path area $\left[\mathrm{m}^{2}\right]$

Aspect ratio, $A R=h / C$

Absolute velocity $[\mathrm{m} / \mathrm{s}]$, or chord length of the airfoil $[\mathrm{mm}$ or $m$ ]

Axial chord length of the airfoil $[\mathrm{mm}$ or $\mathrm{m}]$

Supersonic loss multiplier for profile loss coefficient, $C F M=1+60\left(M_{2_{\text {rel }}}-1\right)^{2}$

Heat capacity at constant pressure $[J /(k g K)]$

Design parameters

Exhaust gas temperature $\left[K\right.$ or $\left.{ }^{\circ} \mathrm{C}\right]$

Fuel-to-air mass flow ratio

Specific total enthalpy $[J / k g]$

Blade span $[m m$ or $m]$

Incidence angle $\left[^{\circ}\right]$

Power deficit index, $I_{P D}=\frac{P W_{\text {exp }}-P W_{\text {measure }}}{P W_{D}}$

Correction factor for impulse blade profile loss correction by Sjolander \& Zhu, associated with Equation (2-35)

Experiential profile loss reduction factor by Kacker \& Okapuu, associated with Equation (2-33)

Roughness loss ratio by Islam \& Sjolander, $K_{L}=\frac{Y_{P R}}{Y_{P}}=\frac{Y_{P}+\Delta Y_{R}}{Y_{p}}$ 
Compressibility correction factor for roughness loss by Jouini

$K_{M}$

et al., $K_{M}=\frac{\Delta Y_{R}(M)}{\Delta Y_{R}(M=0)}=\frac{Y_{P R}(M)-Y_{P}(M)}{\Delta Y_{R}(M=0)}$

Correction factor for nozzle blade profile loss correction by

$K_{N}$

Sjolander \& Zhu, associated with Equation (2-35)

Compressibility correction factor for profile loss,

$K_{p}$

$K_{P}=1-\left(1-K_{1}\right)\left(\frac{M_{1 \text { rel }}}{M_{2_{\text {rel }}}}\right)^{2}$

Correction factor for axial roughness starting position,

$K_{x}$

$K_{x}=\frac{\Delta Y_{R}(x / c=0.05)}{\Delta Y_{R}(x / c)}=f(x / c)$

$k$

$k_{S}$

$M$

$\dot{m}$

$N$

$n$

$o$

$P$

$P R$

PW

Re

$S$

$T$

TIT
Absolute surface roughness height $[\mu m]$

Sand-grain equivalent surface roughness height $[\mu \mathrm{m}]$

Mach number

Mass flow, true $[\mathrm{kg} / \mathrm{s}]$

Shaft speed $[R P M]$

Number of data points

Throat opening [ $\mathrm{mm}$ or $\mathrm{m}]$

Static pressure (or total pressure in the context of GPA analysis) $[P a]$

Pressure ratio

Power $[W$ or $J / s]$

Reynolds number for a turbine blade row, defined in terms of true chord and outlet flow condition of a blade row, $R e=\frac{\rho_{2} V_{2} C}{\mu}$

Blade pitch [ $\mathrm{mm}$ or $\mathrm{m}]$

Temperature $\left[K\right.$ or $\left.{ }^{\circ} \mathrm{C}\right]$

Turbine inlet temperature $\left[K\right.$ or $\left.{ }^{\circ} \mathrm{C}\right]$ 


\section{Greek}

$\alpha$

$\beta$

$\gamma$

$\zeta$

$\eta$

$\theta$

$\Lambda$

$\lambda$

$\rho$

$\tau$
Trailing edge thickness [ $\mathrm{mm}$ or $\mathrm{m}$ ]

Blade velocity $[\mathrm{m} / \mathrm{s}]$

Valve angle $\left[{ }^{\circ}\right]$

Mass flow, corrected, defined as $W=\frac{\dot{m} \sqrt{T_{i}}}{P_{i}}\left[l b / s\left({ }^{\circ} R\right)^{1 / 2} / p s i\right]$

$\mathrm{x}$ coordinate $[\mathrm{mm}$ or $\mathrm{m}]$

Total pressure loss coefficient, see Equation (2-31)

Zweifel coefficient, a characterization for loading of a turbine blade row (compared with an ideal maximum loading) which has significant implications on the performance of a blade row, defined as $Z=2\left(\frac{s}{c_{x}}\right) \cos ^{2} \alpha_{2}\left(\tan \alpha_{2}-\frac{c_{\alpha_{1}}}{c_{\alpha_{2}}} \tan \alpha_{1}\right)$

Flow angle

Airfoil metal angle

Specific heat ratio

Scaling factors for efficiency and mass flow in Equation (2-16) and (2-17), or airfoil stagger angle $\left[^{\circ}\right]$

Isentropic efficiency

Ratio between actual and design values

Turbine stage reaction, defined as $\Lambda=\frac{T_{2}-T_{3}}{T_{02}-T_{03}}$

Spacing parameter, defined as $\lambda=\frac{\text { reference surface area }}{\text { total roughness frontal area }}$

Density $[\mathrm{kg} / \mathrm{s}]$

Tip clearance $[\mathrm{mm}$ or $\mathrm{m}]$ 
$\phi$

$\chi$

$\psi$

$\Omega$

$\omega$

\section{Subscript}

0

1

2

3

A

$a$

air

$b$

baseline

c

calc

ch

cor

$D$, des or on
Flow coefficient, defined as $\phi=\frac{C_{a}}{U}$

Correction factor for $C p_{p}$ in Equation (2-7), or $k_{s} / C \times 10^{3}$ in Equations (2-54) to (2-57)

Work coefficient, defined as $\psi=\frac{\Delta h_{0}}{U^{2}}$

Shaft speed, corrected for compressibility effects $\Omega=\frac{N}{\sqrt{T I T}}$

Shaft speed, uncorrected $[\mathrm{rad} / \mathrm{s}]$

Total condition, distinguished during meanline analysis

Station 1 of a turbine stage, vane inlet, or blade row inlet

Station 2 of a turbine stage, vane outlet and rotor inlet, or blade row outlet

Station 3 of a turbine stage, rotor outlet

Actual condition in Equation (2-16)

Axial component of the velocity triangle

Air working gas

Bleed air

Related to baseline, undegraded condition

Compressor

Predicted parameters (as opposed to measured)

Combustor

Corrected (shaft speed or mass flow)

Value associated with design condition 


\begin{tabular}{ll} 
exh & Exhaust duct \\
Exp or exp & Ideal value corresponding to the input conditions \\
$F$ & Fuel \\
filter & Compressor inlet filter \\
GPA & Related to GPA outcomes \\
$h$ & Hub (radius) \\
$i$ & Inlet, or index for epoch \\
isen & Isentropic (Mach number inferred using isentropic gas relations) \\
loss & Related to loss or entropy generation \\
$m$ & Mean (radius) \\
mech & Mechanical (used for mechanical power loss $P W_{\text {mech }}$ ) \\
measure & Measurement value \\
mix & Mixture with cooling flow (for turbine GPA modelling) \\
$o$ & Outlet of a GTE subsystem \\
on & (On)-design value \\
out & Outlet of blade row \\
$P$ & Profile loss \\
$p$ & Combusted product \\
rel & Toss due to roughness \\
\hline & Reference frame relative to blade row \\
\hline & Turbine, tip (radius) \\
\hline &
\end{tabular}


TC

$\theta$

\section{Superscript}

1

2

3

Acronym

AMDCKO

GTE

GPA

GPP

HP

PHM
Tip clearance loss

Tangential component of the velocity triangle

Value through isentropic expansion, see Equation (2-30) or GPP obtained from the "ideal" GPA model

Station 1 of a turbine stage, vane inlet

Station 2 of a turbine stage, vane outlet, rotor inlet

Station 3 of a turbine stage, rotor outlet

Ainley \& Mathieson, Duncham \& Cane, and Kacker \& Okapuu

Gas turbine engine

Gas path analysis

Gas path parameters

High pressure (turbine)

Prognostic health management 


\section{Chapter 1: Introduction}

\subsection{Background and Motivation}

Gas turbine engines (GTEs) are subjected to various component degradations over the service life, resulting in gradual performance deterioration. Physical degradations of components, reflected by the changes in the operational parameters, such as power output and exhaust gas temperature (EGT), result in decreased efficiency and changes in flow capacity [1]. Degradations can be categorized into two types: short-term recoverable degradation or fouling and long-term non-recoverable degradation. Fouling occurs due to the adhesion of dust particles in the airflow onto the compressor blades. Long-term nonrecoverable degradations are caused by permanent changes of geometry, corrosion, or removal of materials from the components. For both cases, the degradation causes an increase in operational cost, and if not mitigated, could eventually lead to the failure of the components. Diakunchak estimated an excessive annual cost of $\$ 522,000$ for a $46.5 \mathrm{MW}$ GTE, which was assumed to have a conservative 3\% loss in power and $1 \%$ increase in heat rate after 8000 hours of operation, due to unmitigated fouling [2]. In an extreme case, the combination of compressor blade profile changes and airflow distortions caused by fouling can lead to the aerodynamic stall of later stages, and can trigger surge, resulting in blade damage.

While compressor wash can recover the short-term performance deterioration due to fouling, the long-term deteriorations are non-recoverable unless the components are repaired or replaced. Common non-recoverable degradations include erosion, corrosion, and the increase of tip-clearance. Positioned directly downstream of the combustor, the 
high-pressure (HP) turbine vanes and blades are subjected to surface roughening, due to foreign particle deposition, hot corrosion, erosion, and coating spallation [3]. Figure 1-1 shows an example of surface roughness deterioration observed on an HP turbine blade. The surface roughening not only caused additional aerodynamic losses but also increased heat transfer at the blade surface, which reduced the life of the blade. Therefore, in addition to causing performance deterioration, surface roughness accelerates the structural deterioration of vanes and blades, and consequently, reduces remaining usable life.

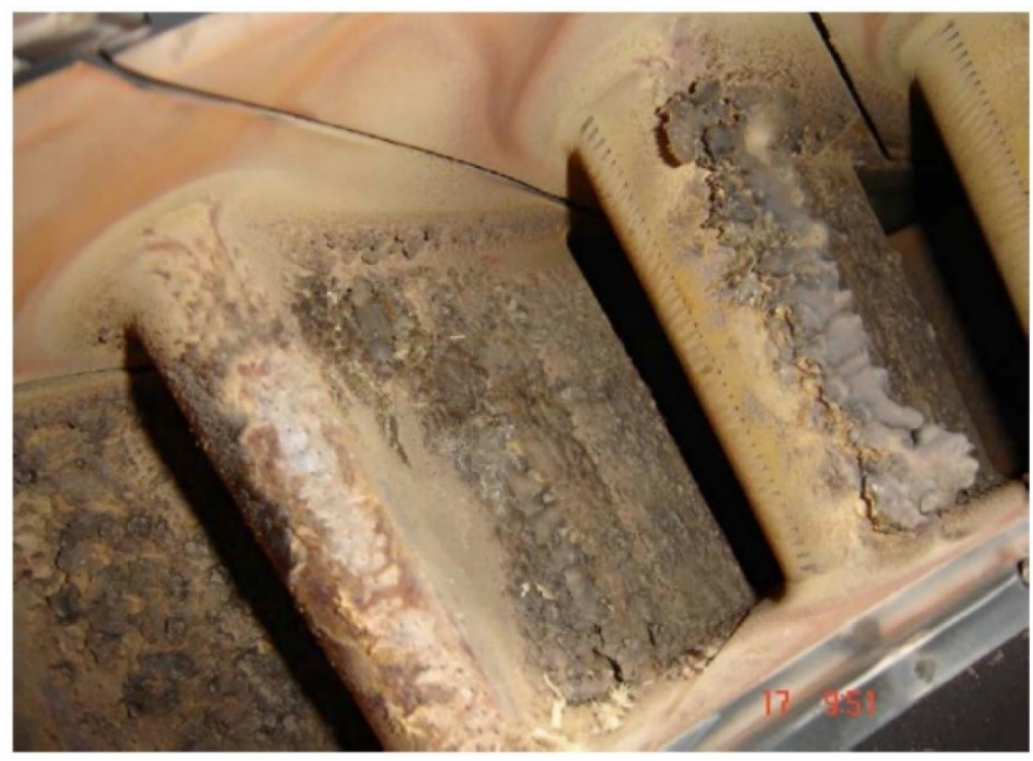

Figure 1-1: Roughened surface of the first stage vane of a utility GTE after 8000 hours of service, adapted from Ref. [4].

Maintenance is scheduled to mitigate degradations, recover performance losses and prevent catastrophic failures. Due to the high-cost associated with downtime, the maintenance philosophy has been shifting from preventive to reliability-centred with the advancement of GTE diagnostic techniques [1]. Regardless, the fault detection methods 
(i.e., physics-based, data-driven, or hybrid) mainly reveal performance deviations or fault indications, and are not sufficient to provide further insights on component-level fault severities, such as surface roughness height and tip-clearance. However, the physical implications revealed by the fault severities are essential for understanding the degradation condition of the critical components, which determines the overhaul schedules. Therefore, to truly attain the cost-saving benefit of reliability-centred maintenance through conditionbased downtime scheduling, online assessment of the physical fault severities is crucial.

Although online techniques are available for tip-clearance measurements, such as borescope photography [5], to the author's best knowledge, there is not any online performance-based monitoring method available in the literature for assessing the severity of the turbine blade and the vane surface roughness, which is usually measured offline using a profilometer [6], [7]. Studies have been conducted to understand the effect of surface roughness on aerodynamic loss and heat transfer, including a series of research programs conducted at Carleton University, including the works by Kind et al. [8], Yuan et al. [9] and Jouini et al. [10]. These contributions, however, have never been utilized in the area of online condition monitoring. Nevertheless, these observations and conceptualizations of the surface roughness effects on aerodynamic losses suggest the potential feasibility of developing an online turbine surface roughness assessment system.

The present work is part of a joint research program between Life Prediction Technologies Incorporated (LPTi) and Carleton University, which aims to develop a practical performance-based prognostic health management (PHM) system for GTEs of basic instrumentations. Figure 1-2 shows the scope of the research objectives with respect 
to GTE health monitoring as well as previous works. Previously, a series of system-level performance diagnostic and prognostic tools had been developed around the basic operational data of an industrial GTE by Hanachi et al. [11], based on a nonlinear gas path analysis (GPA) approach. A description of the data is provided in Section 1.3. As the result, the performance deterioration due to the short-term recoverable compressor fouling, as well as the long-term non-recoverable turbine deterioration could be extracted from the measured operational data. As indicated in Figure 1-2, the present thesis uses the work of Hanachi et al. as its foundation, to further advance health monitoring to the component level, for the motivations described above. The subsequent section elaborates on the objective of this thesis.
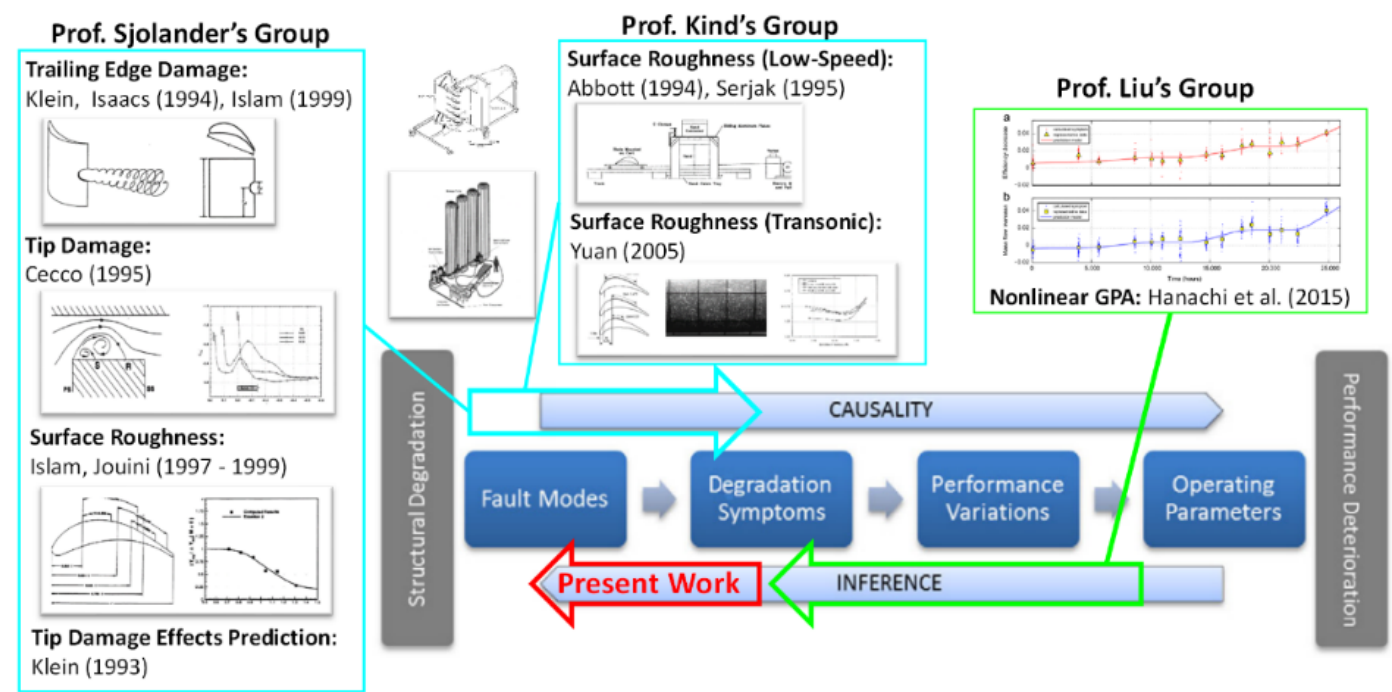

Figure 1-2: Scope of the research objectives with respect to performance-based GTE health monitoring.

\subsection{Thesis Objective}

The objective of the current work is to advance component-level damage assessment using the operational data acquired from basic GTE instrumentation. This was 
accomplished through the development of an online surface roughness assessment framework, which integrated GTE performance deterioration monitoring techniques, as well as an understanding of the aerodynamic performance penalty due to surface roughness of the turbine blades. The goal of the online framework is to determine the turbine surface roughness condition using the operational data presented in Section 1.3.

To achieve this goal, the proposed framework integrates both system-level gas path analysis (GPA) and component-specific turbine meanline analysis. The uncertainties associated with GPA, due to the low sampling rate of the operational data, as well as the modelling assumptions, pose challenges for the extraction of valid information from the results. At the same time, the GPA on turbine degradation is susceptible to compressor fouling effects, due to a lack of instrumentation. A GPA-meanline interfacing process was consequently developed to resolve or alleviate these challenges. A meanline surface roughness identification process was also proposed to establish a meanline model for an existing turbine stage. The process also determined the roughness severity utilizing the empirical roughness loss models developed by previous works.

The present work also evaluated the empirical roughness loss models developed by Islam \& Sjolander [12] and Jouini et al. [10], before integrating the models into the framework. The evaluation was focused on roughness loss prediction under transonic flow conditions, using the wind tunnel study outcomes of Yuan \& Kind [9]. While validating the procedure proposed by Jouini et al. [10], a modification was made to the correlation for the roughness-loss compressibility correction factor, which incorporated the roughness 
position factor. The predictions with the modified correlation showed better agreement with the measured values.

\subsection{The SGT-100 Operational Data}

The operational data used in the present thesis as well as the work of Hanachi et al. were collected from the Ruston/Siemens SGT-100 “Typhoon” GTE. It was in service for power and heat generation in a government plant for three years, which corresponds to the operating period between two major overhauls. The operational data was logged at a rate of one entry every two hours during this period. A set of 44 parameters in each logged entry was comprised of three control inputs, 22 measurements from the auxiliary systems, and 19 measurements from the instrumentations along the gas path. Figure 1-3 shows the plots of the available gas path parameters.

\begin{tabular}{|l|c|c|c|c|c|c|c|}
\hline Source & \multicolumn{4}{|c|}{ GTE operating system } & \multicolumn{3}{c|}{ Environment Canada } \\
\hline Parameter & Power & $\begin{array}{c}\text { Shaft } \\
\text { speed }\end{array}$ & $\begin{array}{c}\text { Inlet } \\
\text { temperature }\end{array}$ & $\boldsymbol{E G T}$ & Temperature & Pressure & $\begin{array}{c}\text { Relative } \\
\text { humidity }\end{array}$ \\
\hline Count & 1 & 1 & 3 & 14 & 1 & 1 & 1 \\
\hline
\end{tabular}
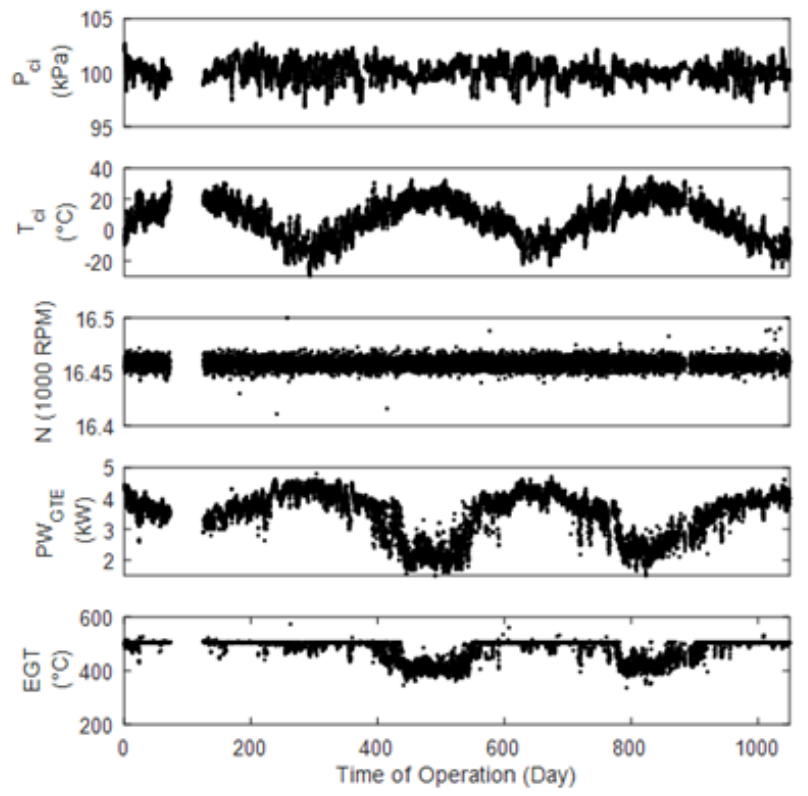

Figure 1-3: Available ambient and gas path measurements, adapted from Ref. [11]. 
For convenience, the detailed description of the SGT-100 engine is provided in Section 5.2.

\subsection{Thesis Outline}

The next chapter provides an overview of the two modelling methods utilized in the framework: the system-level GPA method and the axial-turbine meanline analysis, along with the roughness damage loss correlations employed by the meanline model. The validation of the surface roughness loss correlation is presented in Chapter 3. The developed online-surface roughness framework and its implementation using the SGT-100 operational data are presented in Chapters 4 and 5, respectively. Finally, conclusions and recommendations for future work are summarized in Chapter 6. 


\section{Chapter 2: GTE System and Component Modelling}

\subsection{Introduction}

The current chapter presents the GTE and turbine modelling methods adopted by the proposed online turbine surface roughness assessment framework, which is presented in Chapter 4. The first part of the framework incorporates system-level gas path analysis (GPA), in order to obtain the gas path parameters and performance deterioration "symptoms" of the turbine subsystem from the operating data measured using the basic GTE instrumentation. The second part of the proposed framework utilizes meanline modelling for its low computational cost and minimal required geometric details, while representing the performance of an existing turbine design. The framework can also conveniently incorporate empirical damage-loss models, such as those in [13] and [10], so as to predict the efficiency penalties caused by different faults and associated severities.

The system-level gas path analysis for a single-spool GTE is described first, followed by the meanline analysis for axial turbines. The empirical surface roughness loss correlations are presented as a part of the meanline modelling. The advantages and limitations of both modelling methods for incorporation in the online surface roughness assessment framework are demonstrated and evaluated through application examples. This chapter only intends to provide an overview, while detailed implementation and modification of the methods are described in Chapter 4. 


\subsection{Gas Path Analysis Modelling}

The GPA condition monitoring method utilizes the thermodynamic model and measurement data of the GTE in order to detect and assess the deterioration of various engine modules. The thermodynamic model of the GPA utilizes monitored operating parameters from the GTE's control system; e.g., compressor inlet temperature $\left(T_{c i}\right)$ and pressure $\left(P_{c i}\right)$, shaft speed $(N)$ and fuel mass flow $\left(W_{F}\right)$. It also uses design-defined parameters $(D P)$, including performance maps for the compressor and turbine to predict the corresponding performance parameters of the GTE, such as power $\left(P W_{\text {calc }}\right)$ and exhaust gas temperature $\left(E G T_{\text {calc }}\right)$. As the components of the GTE degrade, the original thermodynamic GTE model no longer represents the condition of the GTE, and the differences between the predicted performance parameters and the measured values increase. Such differences led to the development of various performance deterioration indicators, which aim to capture the degradation symptoms in the GTE.

\subsubsection{Thermodynamic Modelling for a Single Shaft GTE}

A single-shaft GTE follows the Brayton cycle and is comprised of three main modules: compressor, combustor, and turbine. The overall power output of the GTE is the difference between the power extracted by the turbine from the combusted gas $\left(P W_{t}\right)$, and the power consumed during the compression process $\left(P W_{c}\right)$ and through mechanical losses $\left(P W_{\text {mech }}\right)$, which are assumed to be $1 \%$ of the compressor power:

$$
P W_{G T E}=P W_{t}-P W_{c}-P W_{\text {mech }}
$$


The power required by the compressor to achieve a compression ratio of $P R_{c}$ is the product of mass flow $\left(\dot{m}_{c}\right)$, the heat capacity of air $\left(C_{p_{\text {Air }}}\right)$, and the difference between the inlet $\left(T_{c i}\right)$ and outlet $\left(T_{c o}\right)$ total temperatures:

$$
P W_{c}=\dot{m}_{c} C_{p_{A i r}}\left(T_{c o}-T_{c i}\right)
$$

The compressor discharge total temperature is calculated from the inlet temperature and the compression ratio using the isentropic gas relations and the isentropic efficiency of the compressor $\left(\eta_{c}\right)$ :

$$
T_{c o}=T_{c i}\left(\frac{\left(P R_{c}^{\left(\gamma_{a i r}-1\right) / \gamma_{a i r}}-1\right)}{\eta_{c}}+1\right)
$$

where $\gamma_{\text {air }}$ is the specific heat ratio. For an established compressor design, the pressure ratio and compressor efficiency in Equation (2-3) are mainly a function of the corrected mass flow $\left(W_{c_{c o r}}\right)$ and corrected speed $\left(N_{c_{c o r}}\right)$ :

$$
P R_{c}, \eta_{c}=f\left(W_{c}, N_{c_{c o r}}\right)=f\left(\frac{\dot{m}_{c} \sqrt{T_{c i}}}{P_{c i}}, \frac{N}{\sqrt{T_{c i}}}\right)=\operatorname{map}_{c}\left(\frac{\dot{m}_{c} \sqrt{T_{c i}}}{P_{c i}}, \frac{N}{\sqrt{T_{c i}}}\right)
$$

The functional relationship expressed in Equation (2-4) is typically presented in the form of a performance map. Bleed flow $\left(\dot{m}_{b}\right)$ is assumed to be a constant portion of the compressor mass flow that is diverted around the combustion process to cool the turbine components. The mass flow entering the combustion chamber $\left(\dot{m}_{c h}\right)$ is the compressor mass flow excluding the bleed flow:

$$
\dot{m}_{c h}=\dot{m}_{c}-\dot{m}_{b}
$$


Combustion is a chemical process where energy is generated and added into the gas flow. The energy addition manifests as a dramatic increase in the total temperature of the gas at the outlet of the combustor, which can be determined from the first law of thermodynamics:

$$
T_{c h o}=f \frac{C p_{F}\left(T_{F}-T_{c o}\right)+L C V}{(1+f) C p_{p}}+T_{c o}
$$

where $f$ is the ratio of fuel mass flow to combustor mass flow, and $L C V$ is the low calorific value of the fuel. For natural gas fuel, $L C V$ is typically $47000 \mathrm{~kJ} / \mathrm{kg}$. The specific heat capacity of the combustion product $\left(C p_{p}\right)$ is determined as follows:

$$
C p_{p}=C p_{a i r}+f /(1+f) \chi_{C p}
$$

where $\chi_{C p}$ is the correction factor for $C p_{p}$, and it is temperature dependent. The pressure ratio across the combustion chamber $\left(P R_{C h}\right)$ is assumed to be fixed.

The power extracted by the turbine subsystem through expansion over a pressure ratio of $P R_{t}$ is expressed as follows:

$$
P W_{t}=\dot{m}_{t} C p_{\text {mix }}\left(T_{t i}-E G T\right)
$$

where $T_{t i}$ is the turbine inlet temperature and $E G T$ is the exhaust gas temperature. When analyzing a cooled multi-stage turbine at the subsystem level, the cooling effect is added at the inlet boundary condition of the turbine. This results in a model turbine inlet temperature due to the "mixing":

$$
T_{t i}=\frac{\dot{m}_{c h} C p_{p} T_{C h o}+\dot{m}_{b} C p_{a i r} T_{c o}}{\dot{m}_{c h} C p_{p}+\dot{m}_{b} C p_{a i r}}
$$


It is important to note that the $T_{t i}$ from Equation (2-9) does not represent the actual turbine inlet temperature and it is only valid for a turbine with more than one stage, where the cooling air from the first stage affects the energy extraction of the subsequent stage(s). The actual turbine inlet temperature is the combustor outlet temperature and should be used for a turbine with only one stage. The exhaust gas temperature is determined as follows:

$$
E G T=T_{t i}\left(\eta_{t}\left(P R_{t}^{\left(1-\gamma_{m i x}\right) / \gamma_{m i x}}-1\right)+1\right)
$$

where $\gamma_{\text {mix }}$ and $C p_{\text {mix }}$ are for the exhaust gas, which is the mixture of the combusted gas and cooling air. Without considering the effect of humidity, the specific heat capacity of the combusted gas is approximated as:

$$
C p_{\text {mix }}=\frac{\left(1+f-\frac{\dot{m}_{b}}{\dot{m}_{c}}\right) C p_{p}+\frac{\dot{m}_{b}}{\dot{m}_{c}} C p_{c}}{1+f}
$$

Similar to the compressor, the isentropic turbine efficiency in Equation (2-10) is determined through the turbine map along with the corrected turbine mass flow $\left(W_{t}\right)$ :

$$
\frac{\dot{m}_{t} \sqrt{T_{t i}}}{P_{t i}}, \eta_{t}=f\left(P R_{t}, N_{t} \text { cor }\right)=f\left(P R_{t}, \frac{N}{\sqrt{T_{t i}}}\right)=\operatorname{map}_{t}\left(P R_{t}, \frac{N}{\sqrt{T_{t i}}}\right)
$$

Note that the pressure ratio in Equation (2-12) is used as an input to the performance characteristics, as opposed to Equation (2-4), since the parameters in the performance characteristics are inter-dependent. The turbine pressure ratio can be determined through the compressor work, as well as the losses at the intake filter, combustion chamber, and exhaust duct:

$$
P R_{t}=P R_{c} P R_{\text {filter }} P R_{C h} P R_{\text {exh }}
$$


The GPA algorithms can be developed around the thermodynamic models and the instrumentation of the GTE components for both performance prediction and diagnostics. The next section introduces two GPA-based diagnostic models developed previously by Hanachi et al. as part of the research program: "ideal" and "turbine-degrading" models. These two models are incorporated into the proposed framework with modifications, which will be described in Section 4.2

\subsection{2 "Ideal" GPA Model}

The "ideal" model calculates the expected baseline power based on the measured GTE boundary conditions. The expected power is compared with the measured value, in order to capture the performance degradation. The thermodynamic model of the GTE components from Equation (2-1) to (2-13) can be arranged into a mathematically determined system of four independent equations, with four independent variables:

$$
P W_{\text {exp }}=G T E\left(\left[T_{c i}, P_{c i}, E G T, N\right], D P\right)
$$

The output of the model, i.e., the calculated GTE power, is the ideal value corresponding to the input GTE boundary conditions. Although not considered a direct output of the model, it also resolves gas path parameters $(G P P)$ during the solution process. The $G P P$ includes total temperature, pressure, and gas properties at the boundaries of the main subsystems, as well as the mass flow and efficiency of the compressor and turbine.

As the GTE performance deteriorates due to short-term compressor fouling, the compressor becomes less efficient due to the changes in the blade geometry and it consumes a higher portion of the turbine power. The turbine vanes and blades are subjected 
to non-recoverable damages, such as surface roughening, tip-clearance enlargement and trailing edge damage, which permanently impair the turbine's capability to extract energy from the combusted gas flow. In both cases, the monitored power output for the GTE is lower than the expected value for the same operating conditions and exhaust gas temperature. To observe the difference, Hanachi et al. proposed a "power-deficit" index $\left(I_{P D}\right)$, which is defined as follows:

$$
I_{P D}=\frac{P W_{\text {exp }}-P W_{\text {measure }}}{P W_{D}}
$$

where $P W_{\text {measure }}$ and $P W_{D}$ are the measured and design GTE power, respectively.

\subsection{3 "Turbine-degrading" GPA model}

While the ideal GPA model estimates expected baseline power output and compares it with the measured value, the "turbine-degrading" GPA model estimates the variation of the internal state of the turbine due to long-term degradation. The model attributes all long-term degradation in the GTE to the turbine. The model assumes only two turbine degradation "symptoms": the deviations in the isentropic efficiency and mass flow. To calculate the deviations, the isentropic efficiency $\left(\zeta_{\eta}\right)$ and mass flow $\left(\zeta_{W}\right)$ scaling factors are defined:

$$
\begin{gathered}
\zeta_{\eta}=\eta_{t_{D}}-\eta_{t_{A}} / \eta_{t_{D}} \\
\zeta_{W}=W_{t_{A}}-W_{t_{D}} / W_{t_{D}}
\end{gathered}
$$


where $\eta_{t_{A}}$ and $W_{t_{A}}$ are the actual efficiency and mass flow due to degradation, and $\eta_{t_{D}}$ and $W_{t_{D}}$ are the expected values from the turbine map. While $\zeta_{\eta}$ is defined as the decrease in efficiency, $\zeta_{W}$ is defined as the increase in mass flow.

The two scaling factors are directly applied to the turbine performance characteristics output: the corrected mass flow and efficiency in Equation (2-12). With the introduction of two additional unknowns, the original arrangement of the system expressed as Equation (2-14), becomes indeterminate. Two additional independent GPPS are required to establish two additional independent equations, such that the number of equations matches the number of unknowns. For the present case, the measured power and fuel valve angle $(V A)$ are added as independent inputs to the model, which yields the following expression for the turbine-degrading GPA model:

$$
\left[\zeta_{\eta}, \zeta_{W}\right]=G T E\left(\left[T_{c i}, P_{c i}, N, E G T, P W_{\text {measure }}, V A\right], D P\right)
$$

where the fuel valve angle is used to estimate the pressure of the combustion chamber.

Both $\zeta_{\eta}$ and $\zeta_{W}$ are directly used as performance deterioration indicators for the turbine subsystem in the GPA model. Equations (2-16) and (2-17) are used to calculate the actual turbine efficiency and mass flow corresponding to the input measurement variables. However, the present turbine-degrading model is susceptible to the effect of degradation in non-turbine components, such as compressor fouling, which yields predictions that are physically invalid. Such shortcoming will be examined in the subsequent section using an application case. 


\subsubsection{Remarks on GPA Modelling}

Both the ideal and the turbine-degrading GPA method demonstrated effectiveness in detecting and assessing the degradations in the GTE. Figure 2-1 shows the results of the both GPA models applied to the three-year operating data of the "Typhoon" GTE, which was described in Section 1.3. The gap between days 72 and 124 is due to the shutdown of the GTE during the summer, as indicated on the operational log.

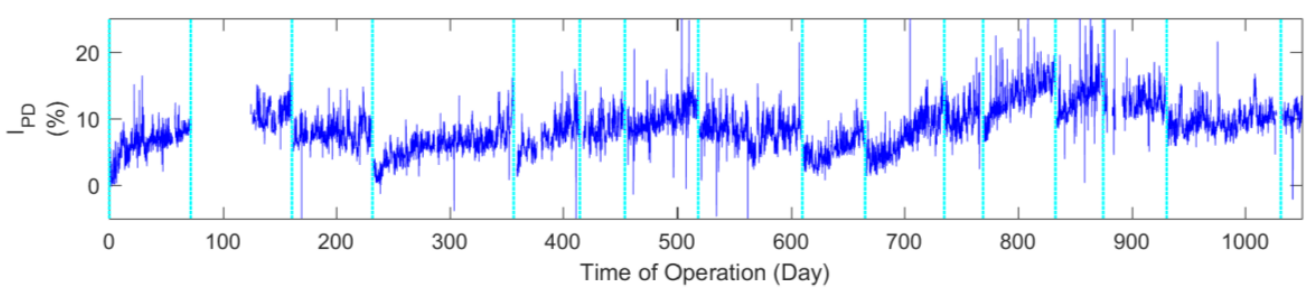

(a)
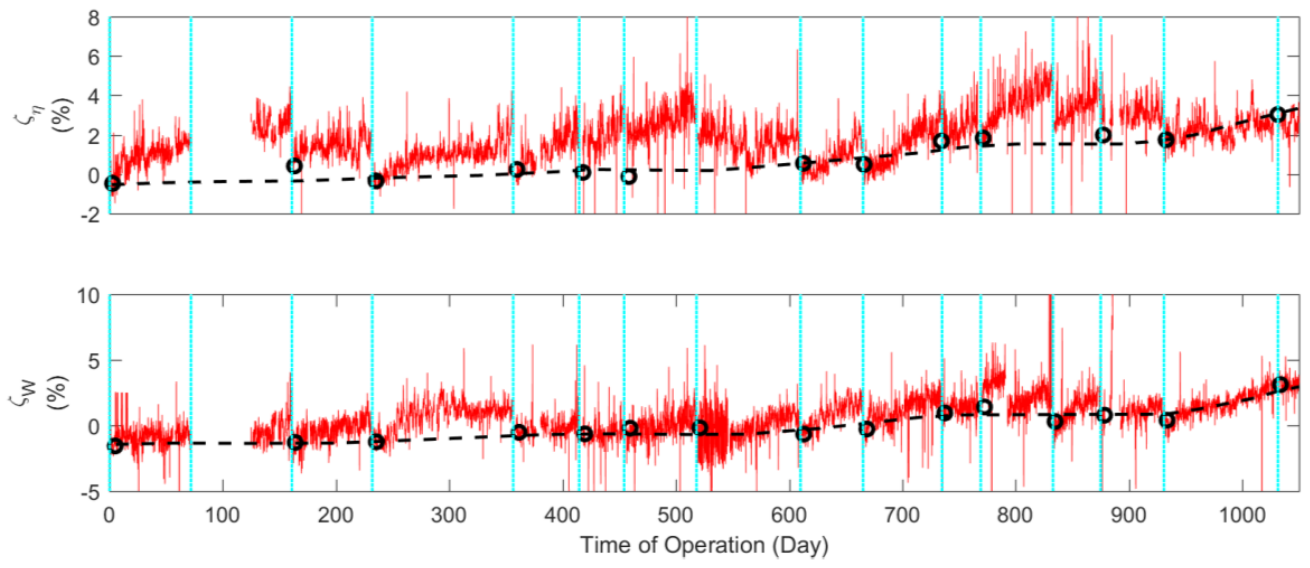

- Median value within five days (60 entries)

- - Fitted curve by Hanachi et al.

(b)

Recorded compressor wash times from the operational log

Figure 2-1: Three years of GPA health monitoring of operational data from the Typhoon GTE: (a) the $I_{P D}$ index by the ideal model, and (b) the isentropic efficiency decrease and mass flow increase of the turbine subsystem assessed by the turbine-degrading model.

As seen in Figure 2-1(a), the power deficit indicator, $I_{P D}$, estimated with the ideal GPA model using Equation (2-15), varied in a tooth-saw pattern. The points of the sudden- 
falls correspond exactly to the recorded compressor washes, which in turn, demonstrates the effectiveness of the $I_{P D}$ indicator in capturing short-term compressor fouling. The differences in the starting values of $I_{P D}$ after each wash were due to the quality of the wash, as well as the severity of the long-term degradation of the components.

Figure 2-1(b) shows the long-term turbine degradation "symptoms," $\zeta_{\eta}$ and $\zeta_{W}$, estimated using the turbine-degrading model. The compressor fouling indeed influenced both $\zeta_{\eta}$ and $\zeta_{W}$, which also varied in tooth-saw patterns. To minimize the influence of compressor fouling on the assessment of turbine degradation, Hanachi et al. decreased sampling duration to a five-day period (60 entries) after each wash based on experience [14]. As a system-level performance deterioration estimation, the $\zeta_{\eta}$ and $\zeta_{W}$ trends were established using the median value of the sample without distinguishing the operating conditions. Hanachi et al. correlated the growth of the degradation symptoms with power and time using the identified median values, which resulted in the degradation-state evolution models plotted as dashed lines in Figure 2-1(b).

Figure 2-2 shows the gas path parameters associated with the turbine subsystem, estimated by both the ideal model and the turbine-degrading model. The estimated $P_{t i}, P R_{t}$ and $\dot{m}_{t}$ of both models showed adequate agreement. The tooth-saw patterns were present in the $T_{t i}, W_{t}$, and $\eta_{t}$ estimated by the turbine-degrading model, indicating the influence of compressor fouling on these values. A close examination of the turbine-degrading model suggested the following plausible mechanism through which compressor fouling affects the model's GPP estimation: 
(1) The turbine-degradation assessing algorithm attributes all the degradation effects to the turbine subsystem, and therefore does not account for the increased power consumption by the compressor with decreased efficiency due to fouling.

(2) Physically, as the compressor fouling effect becomes significant, the compressor consumes a higher portion of the power generated by the turbine, causing the recorded GTE power to deviate from the expected values.

(3) The EGT, however, is not affected by fouling, since it is taken directly from the measurement and used as one of the convergence criteria, as seen in Equation $(2-18)$.

(4) Consequently, while assuming undegraded compressor performance, the algorithm of the turbine-degrading GPA method matches the recorded EGT and GTE power by accounting for less turbine power extraction, which yields underestimated $T_{t i}$, and unrealistic $\eta_{t}$ and $W_{t}$. 

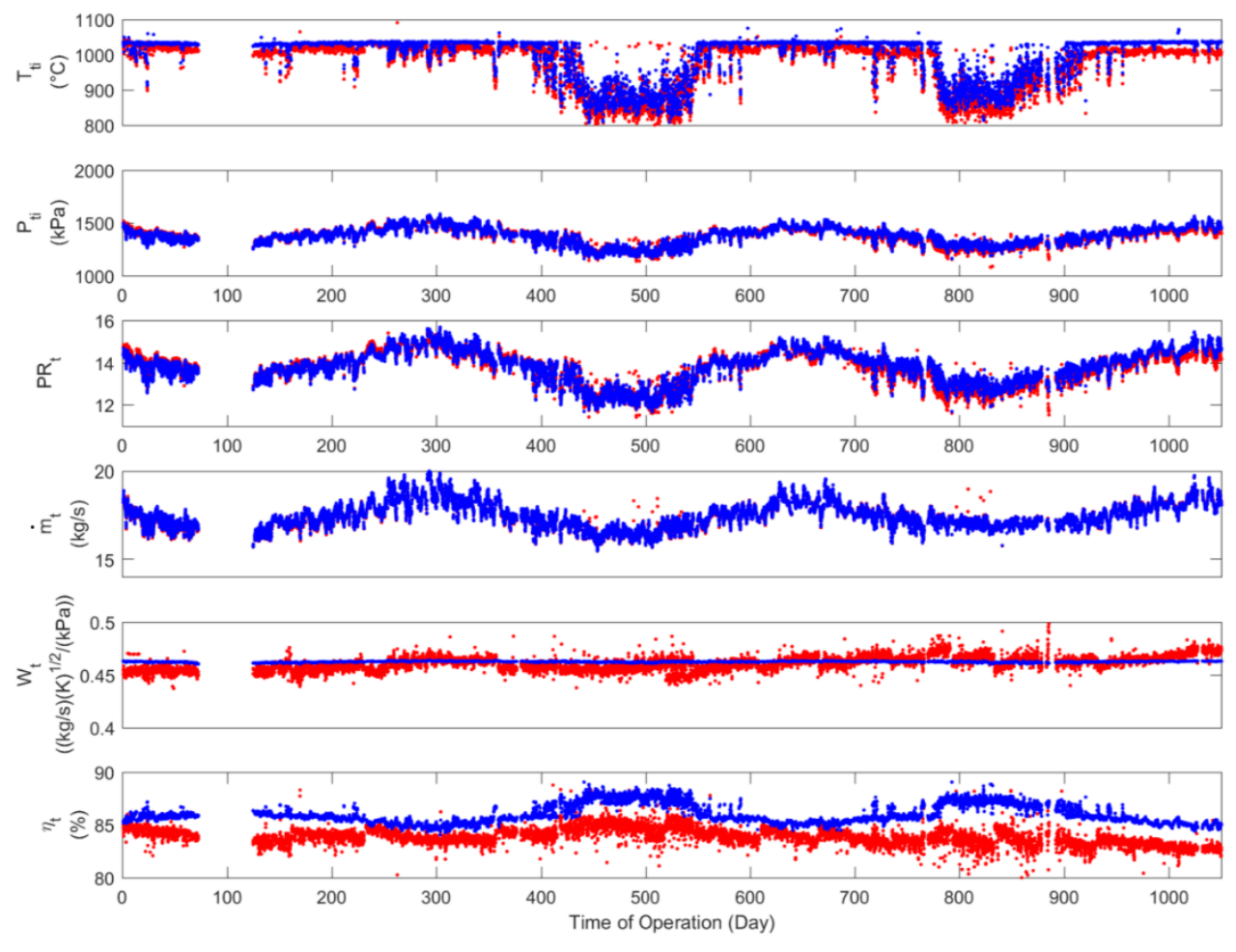

- Ideal Model

- Turbine-degrading Model

Figure 2-2: Gas path parameters estimated by both the ideal and the turbine-degrading GPA model.

From the perspective of GTE condition monitoring, both the ideal and turbinedegrading models demonstrated effectiveness in capturing the short-term compressor fouling and long-term turbine performance deterioration, respectively. However, to be used as a part of the component-specific fault severity assessment framework, the following limitations and challenges must be addressed:

(1) As seen in Figure 2-2, the GPA results usually possess a significant amount of noise, due to the measurement uncertainties, the thermodynamic modelling assumptions and numerical errors. While the noise level is tolerable for performance monitoring, it can lead to divergence of the solution when applied to 
the component-specific models, such as the turbine meanline model presented in the subsequent section, which has a higher demand for valid input data.

(2) For the same severity level of a fault, such as a specific blade surface roughness height, the associated efficiency loss can vary dramatically under different operating conditions. While the GPA method does not necessarily consider such an effect for mere fault indication purposes, component-based modelling requires clear distinction of operating conditions and associated effects, so as to accurately assess the fault severity.

(3) In the turbine-degrading model, the short-term compressor fouling effect is usually identified and alleviated by only considering the data within a limited period after each compressor wash, where the fouling effect is at a minimum. Although Hanachi et al. suggested a five-day sampling period after each wash to alleviate the fouling effect, the value was based on the authors' experience and does not apply to GTEs operated in different environments. In reality, the fouling rate is dependent on the environmental conditions as well as the wash quality, which vary over time. A procedure is still required to determine a suitable sampling period, in which the fouling effect is a minimum, and the data quantity is sufficient to address the challenges mentioned in (1) and (2).

While utilizing both the ideal and the turbine-degrading GPA models according to inherent strengths, the proposed surface roughness assessment framework attempts to alleviate the aforementioned limitations and challenges associated with the GPA methods through an epoch-based sampling process. This is presented in Section 4.2 . 


\subsection{Axial-Turbine Meanline Modelling}

The meanline aerodynamic modelling method was originally a low-fidelity design and performance analysis tool used in the preliminary development phase. It determined key turbomachinery parameters prior to more detailed high-fidelity design and analysis [15]. The meanline turbine model analyzes flow conditions at the mean radius of the vane or blade, which is assumed to be a valid representation of the flow across the full span. The mean radius is defined as the radius that divides the gas path area in half:

$$
r_{m}=\sqrt{\frac{1}{2}\left(r_{h}^{2}+r_{t}^{2}\right)}
$$

where $r_{m}, r_{h}$, and $r_{t}$ are the mean radius, hub radius, and tip radius, respectively. The meanline model estimates flow conditions at the mean radii using velocity triangles and empirical correlations, in order to attain preliminary performance estimations of a turbine design, which typically includes machine size and rotational speed.

\subsubsection{Meanline Modelling of an Axial-Turbine Stage}

The meanline model is governed by the conservation of mass and the Euler turbine equation (i.e., the conservation of energy) as follows:

$$
\begin{gathered}
\dot{m}=\rho A C_{a} \\
\Delta h_{0_{\text {stage }}}=\omega\left(r_{3} C_{\theta_{3}}-r_{2} C_{\theta_{2}}\right)
\end{gathered}
$$

where $\dot{m}$ is the mass flow rate, $\rho$ is the gas density, $A$ is the annulus area of the gas path, $\Delta h_{0_{\text {stage }}}$ is the specific total enthalpy change due to energy transfer between the rotor and 
the fluid, $\omega$ is the rotation speed, $r$ is the mean radii, and $C_{a}$ and $C_{\theta}$ are the axial and tangential flow velocity, respectively, as shown in Figure 2-3. The subscripts " 2 " and "3" indicate the inlet and outlet location of the rotor, respectively, as shown in Figure 2-3. The stagnation specific enthalpy extracted by a turbine stage is related to the stagnation temperature drop as follows:

$$
\Delta h_{0_{\text {stage }}}=C p \Delta T_{0}=C p\left(T_{01}-T_{03}\right)
$$

The velocity components in Equations (2-20) and (2-21) are determined with the establishment of velocity triangles. As seen in Figure 2-3, a velocity triangle relates the absolute flow velocity $(C)$ to the relative flow velocity $(W)$, which is defined with respect to the rotating blade section at the mean radius. The meanline blade velocity $(U)$ is defined as:

$$
U=\omega r_{m}
$$

The establishment of the velocity triangles determines both the absolute and the relative gas conditions at each one of the three stations defined in Figure 2-3. The changes of state due to the expansion process across the turbine stage can be visualized in the temperature vs. specific entropy diagram (or T-s diagram) in Figure 2-4. The relationship between stagnation temperature and static temperature, shown in the T-s diagram, is interchangeable with the following form:

$$
\frac{T_{0}}{T}=1+\left(\frac{\gamma-1}{2}\right) M^{2}
$$

where $\gamma$ is the specific heat ratio of the working fluid (i.e., the combusted gas), $M$ is the Mach number of the flow, and the subscript ' 0 ' indicates the total condition. 


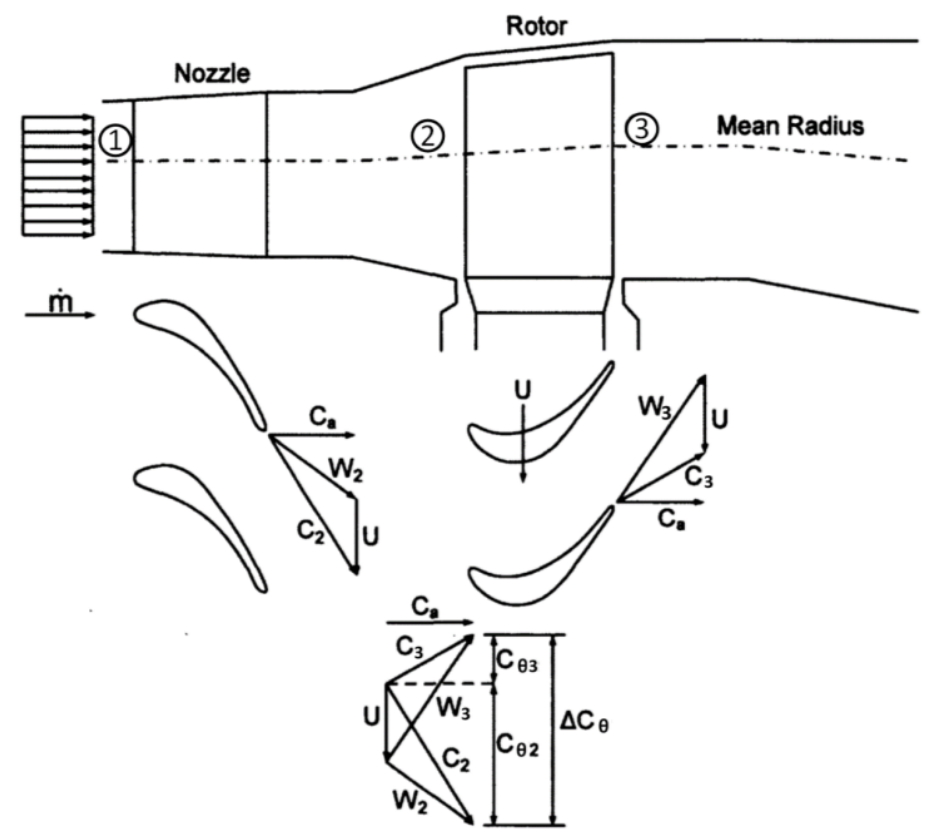

Figure 2-3: Station numbering and illustrations of velocity triangles for a single turbine stage, adapted from Ref. [16].

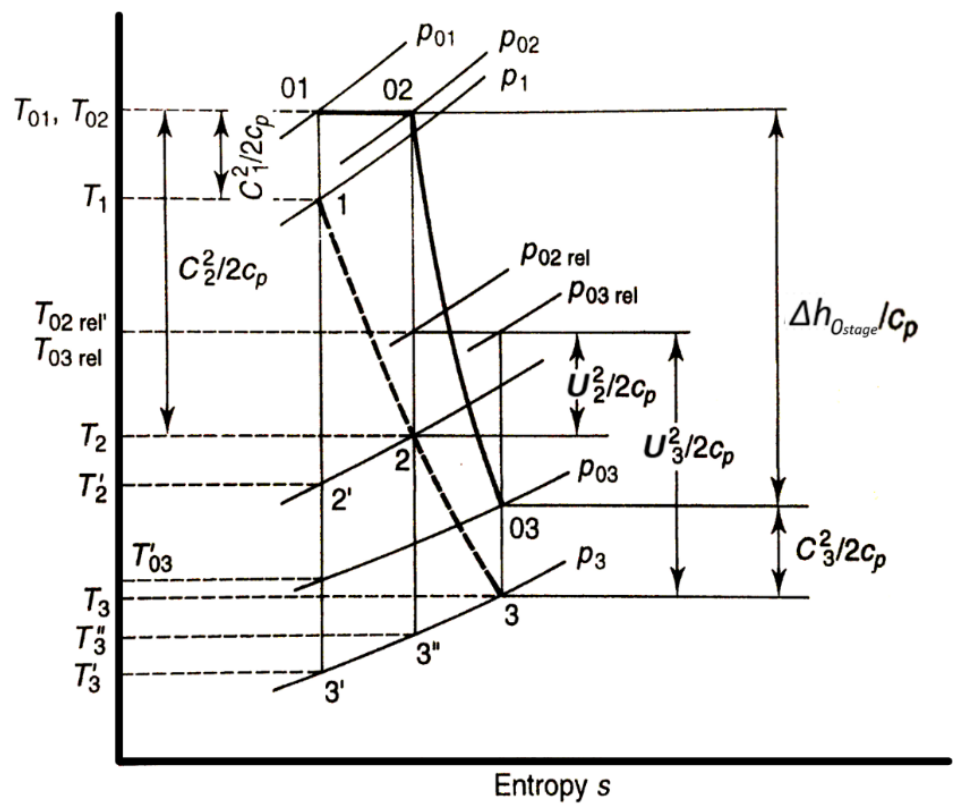

Figure 2-4: The expansion process across a turbine stage illustrated on a T-s diagram, adapted from Ref. [17]. 
Since, in most cases, the working fluid of a turbine is a compressible ideal gas, the following isentropic relationship between the static and stagnation pressure is used:

$$
\frac{P_{0}}{P}=\left(1+\left(\frac{\gamma-1}{2}\right) M^{2}\right)^{\gamma /(\gamma-1)}=\left(\frac{T_{0}}{T}\right)^{\gamma /(\gamma-1)}
$$

After pinpointing the velocity triangle (flow angles) and the annulus area at each location indicated in Figure 2-3, detailed meanline blade row parameters, such as the optimum solidity (pitch-to-chord ratio), are designed with the guidance of empirical correlations, such as the Zweifel coefficients. Metal angles, stagger angles, and maximum thickness are optimized by examining aerodynamic losses. The T-s diagram shows the effect of irreversibility, or losses, in the form of a total pressure drop across the blade row, which is caused by an increase in entropy during the thermodynamic process. In meanline modelling, the losses are usually assessed using empirical correlations, which are presented in the subsequent section.

The main performance parameters for a turbine stage include flow coefficient $\left(\phi_{\text {stage }}\right)$, work coefficient $\left(\psi_{\text {stage }}\right)$, stage reaction $\left(\Lambda_{\text {stage }}\right)$, pressure ratio $\left(P R_{\text {stage }}\right)$ and total-to-total isentropic efficiency $\left(\eta_{\text {stage }}\right)$, which are defined as follows:

$$
\begin{gathered}
\phi_{\text {stage }}=\frac{C_{a}}{U} \\
\psi_{\text {stage }}=\frac{\Delta h_{0}}{U^{2}} \\
\Lambda_{\text {stage }}=\frac{T_{2}-T_{3}}{T_{02}-T_{03}} \\
P R_{\text {stage }}=\frac{P_{01}}{P_{03}}
\end{gathered}
$$




$$
\eta_{\text {stage }}=\frac{T_{01}-T_{03}}{T_{01}-T_{03}{ }^{\prime}}
$$

These parameters can be used either as design points or for evaluating the performance of the design.

\subsubsection{Empirical Loss Correlations}

The pressure and velocity gradients in the axial turbine flow develop various flow features, some of which reduce the efficiency and power output of the turbine and are referred to as "losses." These losses are manifested as an increase in entropy, as the flow passes through the turbine blade row. The losses are typically divided into the following components, based on distinctive mechanisms: profile loss, secondary loss, annulus loss, and tip-clearance loss [18]. These losses are typically interdependent in reality.

Empirical correlations, such as those published in [19], were developed to account for aerodynamic losses. Since entropy cannot be measured directly, it is more convenient to express losses in terms of measurable quantities, such as the total pressure drop, as seen in the T-s diagram in Figure 2-3. Many internal flow empirical loss models use the total pressure loss coefficient $(Y)$. For an axial turbine cascade, $Y$ is usually defined by the outlet dynamic pressure:

$$
Y=\frac{\Delta P_{0_{\text {loss }}}}{P_{0_{\text {out }}}-P_{\text {out }}}
$$

where $\Delta P_{0_{\text {loss }}}$ is the total pressure loss through an irreversible process. The loss coefficients are correlated with the inlet and outlet flow and blade angles, as well as key airfoil 
geometries, such as solidity and maximum thickness-to-chord ratio. After accounting for all the loss components and converging on the gas properties, the efficiency of the turbine stage can be calculated using Equation (2-30).

Various empirical loss systems have been previously proposed, including Ainley \& Mathieson [20], Craig \& Cox [21], and Baljé \& Binsley [22]. Among these, the Ainley \& Mathieson is the most widely used axial turbine loss system, with various improvements having been made over time. The remainder of this section presents the loss system employed by the present thesis: the Ainley \& Mathieson, Dunham \& Came, Kacker \& Okapuu (AMDCKO) system with an improved profile loss correlation by Zhu \& Sjolander [23]. This particular system was chosen for the meanline modelling of the present work, due to the availability of various damage loss correlations developed around such systems. Examples include the trailing edge damage correlations by Isaacs \& Sjolander [24] and the surface roughness correlation system by Jouini, Islam \& Sjolander [10], which was adapted for the present thesis. The AMDCKO loss system divides loss components as follows:

$$
Y_{\text {combined }}=Y_{P} f(R e) C F M+Y_{T E T}^{\prime}+Y_{S}+Y_{T C}
$$

where $Y_{P}, Y_{S}, Y_{T E T}^{\prime}$, and $Y_{T C}$, are the total pressure loss coefficients for profile, secondary, trailing edge, and tip clearance loss components, respectively. $f(R e)$ and $C F M$ are the correction factors for Reynolds number and supersonic drag, respectively.

The profile loss assessment for a turbine airfoil, with the key geometric parameters defined in Figure 2-5, is conducted using a weighted-average approach. The profile loss of a turbine airfoil is assessed based on the empirically correlated profile loss coefficients of two extreme cases of blade design: the impulse blades $\left(\Lambda=0, \beta_{1}=\alpha_{2}\right)$ through which 
flow is not accelerated, and the reaction blades $\left(\Lambda=50, \beta_{1}=0^{\circ}\right)$ through which flow achieves maximum acceleration. The profile loss correlation is expressed as follows:

$$
Y_{P}=0.914\left(K_{\bmod } Y_{P, A M} K_{P}+Y_{S H O C K}\right)
$$

where 0.914 is the factor applied to remove the trailing edge loss from the original Ainley \& Mathieson model, which includes the loss associated with a trailing edge thickness-tochord ratio $(t / C)$ of $0.2 . K_{m o d}$ is the "experience" factor proposed by Kacker \& Okapuu to account for the reduction in profile loss in more modern and optimized blade designs, which have advanced from the time the Ainley \& Mathieson correlation was introduced. Based on the experimental data of 33 contemporary turbine designs, Kacker \& Okapuu suggested a value of $2 / 3$ for the experience factor. $Y_{P, A M}$ is the weighted-average profile loss model by Ainley \& Mathieson, which is expressed as follows:

$$
Y_{P, A M}=\left\{Y_{P,\left(\beta_{1}=0\right)}+\left(\frac{\beta_{1}}{\alpha_{2}}\right)^{2}\left[Y_{P,\left(\beta_{1}=\alpha_{2}\right)}-Y_{P,\left(\beta_{1}=0\right)}\right]\right\}\left(\frac{t_{\max } / C}{0.2}\right)^{\beta_{1} / \alpha_{2}}
$$

where $Y_{P,\left(\beta_{1}=\alpha_{2}\right)}$ and $Y_{P,\left(\beta_{1}=0\right)}$ are the profile loss coefficients for the impulse and reaction blades, respectively. The empirical correlations for $Y_{P,\left(\beta_{1}=\alpha_{2}\right)}$ and $Y_{P,\left(\beta_{1}=0\right)}$ are shown in Figure 2-6.

Zhu \& Sjolander found that the profile loss predictions of axial inflow nozzles by the Kacker \& Okapuu's model were optimistic, and proposed the following variant of $Y_{P, A M}:$

$$
Y_{P, A M}=\left\{K_{N} Y_{P,\left(\beta_{1}=0\right)}+\left(\frac{\beta_{1}}{\alpha_{2}}\right)^{2}\left[K_{I} Y_{P,\left(\beta_{1}=\alpha_{2}\right)}-K_{N} Y_{P,\left(\beta_{1}=0\right)}\right]\right\}\left(\frac{t_{\max } / C}{0.2}\right)^{K_{m} \beta_{1} / \alpha_{2}}
$$

with: 


$$
\begin{gathered}
K_{m}= \begin{cases}1 & \text { for } t_{\max } / C \leq 0.2 \\
-1 & \text { for } t_{\max } / C>0.2\end{cases} \\
K_{N}=0.825, K_{I}=2 / 3
\end{gathered}
$$

When applying the modified $Y_{P, A M}, K_{\text {mod }}$ in Equation (2-33) is set to one. The profile loss predicted with the modified model displayed a suitable fit to the empirical data, especially for the blades with a maximum thickness-to-chord ratio greater than 0.2 .

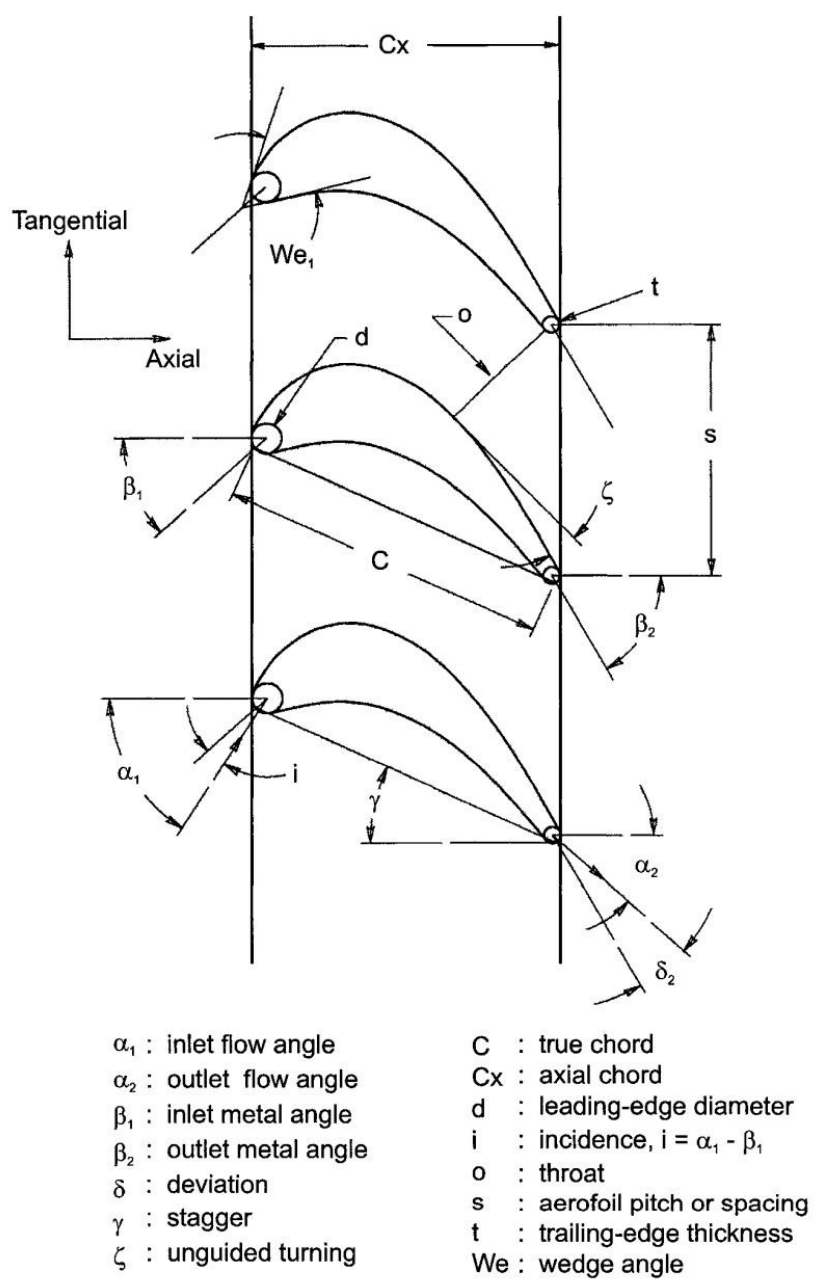

Figure 2-5: Definition of a turbine airfoil, adapted from Ref. [25]. 


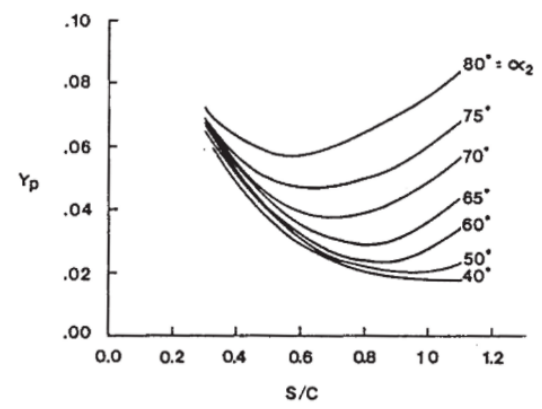

a. Nozzle Blades, $\beta 1=0^{\circ}, \mathrm{t}_{\max } / \mathrm{C}=0.2$

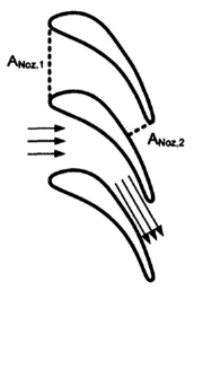

(1)

\begin{abstract}
Figure 2-6: The Ainley \& Mathieson profile loss coefficient data for: a. nozzle blades, and b. impulse blades with a maximum thickness to chord ratio $\left(t_{\max } / \boldsymbol{C}\right)$ of 0.2 , adapted from Ref. [16] and [19].
\end{abstract}

Kacker \& Okapuu introduced means to correct for compressibility effects. The Ainley \& Mathieson correlations in Figure 2-6 were based on the test data from a lowspeed cascade. Thus, the compressibility effects, such as the thinning of the boundary layer and suppression of flow separation, were not accounted for in the correlation when applied to turbine stages of higher flow coefficient. The compressibility correction term, $K_{P}$, in Equation (2-33) accounts for the beneficial compressibility effects, and is defined with the inlet and outlet Mach number relative to the blade row:

$$
K_{P}=1-\left(1-K_{1}\right)\left(\frac{M_{1_{r e l}}}{M_{2_{r e l}}}\right)^{2}
$$

where $K_{1}$ is defined as:

$$
K_{1}=1-0.625\left[M_{2_{r e l}}-0.2+\left|M_{2_{r e l}}-0.2\right|\right]
$$

The $Y_{\text {SHOCK }}$ term in Equation (2-33) accounts for the losses due to the shock waves at the leading edge of the hub stream surface, where the Mach number is typically higher than at the midspan. $Y_{\text {SHOCK }}$ is defined by Kacker \& Okapuu as follows: 


$$
Y_{\text {SHOCK }}=\left(\frac{\Delta P_{0}}{q_{1}}\right)\left(\frac{P_{1}}{P_{2}}\right) \frac{1-\left(1+\frac{\gamma-1}{2} M_{1_{\text {rel }}}^{2}\right)^{\gamma /(\gamma-1)}}{1-\left(1+\frac{\gamma-1}{2} M_{2_{\text {rel }}}^{2}\right)^{\gamma /(\gamma-1)}}
$$

Kacker \& Okapuu proposed the following correction, based on a reference Reynolds number of $2 \times 10^{5}$ :

$$
f(R e)=\left\{\begin{array}{cl}
\left(\frac{R e}{2 \times 10^{5}}\right)^{-0.4} & \text { for } R e \leq 2 \times 10^{5} \text { (laminar) } \\
1.0 & \text { for } 2 \times 10^{5}<R e<1 \times 10^{6} \text { (transitional) } \\
\left(\frac{R e}{1 \times 10^{6}}\right)^{-0.2} & \text { for } R e \geq 1 \times 10^{6} \text { (turbulent) }
\end{array}\right.
$$

The Reynolds number was defined in terms of true chord and exit gas conditions. Since the true variation of loss coefficient with Reynolds number is a function of blade geometry, this correction only serves as a rough approximation.

The AMDCKO system formulates the trailing edge loss coefficient through a weighted-average approach, similar to the correlation method for the profile loss. The trailing edge losses, in the form of energy loss coefficients $\left(\Delta \phi_{T E T}^{2}\right)$, are correlated for both the nozzle and the impulse blade as a function of the trailing edge thickness-to-throat ratio $(t / o)$. The plot of the correlations is shown in Figure 2-7. 


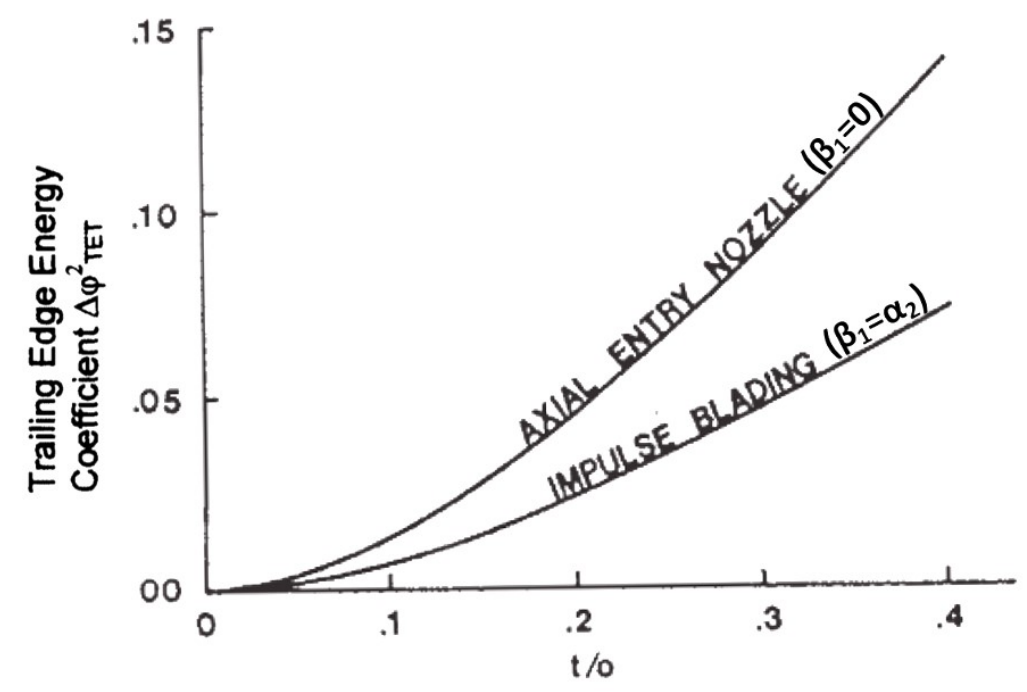

Figure 2-7: Trailing edge energy loss coefficients for the nozzle and impulse blades, adapted from Ref. [19].

The trailing edge energy loss correlation for any given blade geometry lies between a nozzle and an impulse blade, and is determined using the following expression:

$$
\Delta \phi_{T E T}^{2}=\Delta \phi_{T E T\left(\beta_{1}=0\right)}^{2}+\left|\frac{\beta_{1}}{\alpha_{2}}\right|\left(\frac{\beta_{1}}{\alpha_{2}}\right)\left[\Delta \phi_{T E T\left(\beta_{1}=\alpha_{2}\right)}^{2}-\Delta \phi_{T E T}^{2}{ }_{\left(\beta_{1}=0\right)}\right]
$$

Kacker \& Okapuu provided the following expression to convert the energy loss coefficient from Equation (2-40) into a form of total pressure coefficient:

$$
Y_{T E T}^{\prime}=\frac{\left[1-\frac{\gamma-1}{2} M_{2_{\text {rel }}}^{2}\left(\frac{1}{1-\Delta \phi_{T E T}^{2}}-1\right)\right]^{-\frac{\gamma}{\gamma-1}}-1}{1-\left(1+\frac{\gamma-1}{2} M_{2_{\text {rel }}}^{2}\right)^{-\frac{\gamma}{\gamma-1}}}
$$

For a discharge Mach number greater than unity, the AMDCKO uses the following multiplier to profile loss, so as to account for losses due to supersonic drag:

$$
C F M=1+60\left(M_{2_{r e l}}-1\right)^{2}
$$


Kacker \& Okapuu noted that the $C F M$ was a major shortcoming of the loss system, as it did not take into account the blade geometry, and consequently neglected the supersonic drag rise due to the limit load of the airfoil.

The secondary losses and the tip-clearance loss prediction utilize the correlations of the AMDCKO system. The secondary loss coefficient is formulated as follows:

$$
Y s=1.2\left[0.0334 f_{(A R)}\left(\frac{\cos \alpha_{2}}{\cos \beta_{1}}\right)\left(\frac{C_{L}}{s / C}\right)^{2}\left(\frac{\cos \alpha_{2}^{2}}{\cos \beta_{1}^{2}}\right)\right] K_{S}
$$

where $C_{L}$ is the flow lift coefficient defined using the flow turning of the blade row:

$$
C_{L}=\tan ^{-1}\left[\frac{1}{2}\left(\tan \alpha_{1}+\tan \alpha_{2}\right)\right] \cos \alpha_{m}
$$

$\alpha_{m}$ is the mean flow angle defined as:

$$
\alpha_{m}=\tan ^{-1}\left[\frac{1}{2}\left(\tan \alpha_{1}-\tan \alpha_{2}\right)\right]
$$

$f_{(A R)}$ is the correction factor used to account for the effect of the aspect ratio $(h / C)$, which is defined as the ratio between blade height $(h)$ and the true chord length at the mean radius (C). $f_{(A R)}$ is correlated for two ranges of aspect ratio:

$$
f_{(A R)}= \begin{cases}\frac{1-0.25 \sqrt{2-h / C}}{h / C} & \text { for } h / C \leq 2 \\ \frac{1}{h / C} & \text { for } h / C>2\end{cases}
$$

$K_{S}$ captures the compressibility effects for subsonic Mach numbers, and is defined as follows: 


$$
K_{S}= \begin{cases}1-\left(\frac{1}{h / C_{x}}\right)^{2}\left(1-K_{p}\left(M_{2_{r e l}}\right)\right) & \text { for } M_{2_{r e l}} \leq 1 \\ 1-\left(\frac{1}{h / C_{x}}\right)^{2}\left(1-K_{p}\left(M_{2_{\text {rel }}}=1\right)\right) & \text { for } M_{2_{\text {rel }}}>1\end{cases}
$$

$K_{p}$ is the compressibility correction factor for profile loss, as defined in Equation (2-36).

The tip-clearance loss coefficient is correlated differently for the shrouded and unshrouded rotor blades. For shrouded blades, the specific tip-clearance loss $(\tau)$ is correlated as follows:

where

$$
Y_{T C}=0.37 \frac{C}{h}\left(\frac{\tau^{\prime}}{C}\right)^{0.78}\left(\frac{C_{L}}{s / C}\right)^{2} \frac{\cos \alpha_{2}^{2}}{\cos \alpha_{m}^{3}}
$$

$$
\tau^{\prime}=\frac{\tau}{(\text { number of seals })^{0.42}}
$$

For unshrouded blades, the tip-clearance loss is correlated in terms of the variation of the overall efficiency $(\Delta \eta)$, as a result of the changes in tip clearance $(\Delta \tau)$ :

$$
\frac{\Delta \eta}{\eta_{(\tau=0)}}=0.93\left(\frac{\Delta \tau}{h \cos \alpha_{2}} \frac{r_{t}}{r_{m}}\right)
$$

where $\eta_{(\tau=0)}$ is the turbine efficiency without accounting for tip-clearance loss. Kacker \& Okapuu claimed that the available experimental data correlated to within $\pm 15 \%$ using Equation (2-50). Since the overall loss system is the linear summation of the total pressure loss coefficients of each loss component, as expressed in Equation (2-32), $\Delta \eta$ obtained from Equation (2-50) cannot be directly applied. $\Delta \eta$ affects the gas properties and consequently the velocity triangles, which determine most of the loss components and $\eta_{(\tau=0)}$. Thus, Equation (2-50) is resolved iteratively along with the velocity triangles and other loss components. 
The recent improvements to the correlations for both the secondary loss and the tipclearance loss include the work of Benner et al. [25], Yaras \& Sjolander [26], and Aungier [27]. Since the work presented in this thesis is at the level of concept validation, these improvements have not yet been implemented. Therefore, detailed descriptions of these improvements are not provided.

\subsubsection{Remarks on Meanline Analysis of Axial Turbines}

As mentioned previously, meanline turbine aerodynamic modelling has been used for the preliminary design of key turbine flow and geometric parameters, such as annuli geometry and blade angles. It is used during the aerodynamic conceptual design phase for its simplicity and reasonably accurate design performance prediction, especially when analyzing turbines of high hub-to-tip ratios.

Although meanline turbomachinery modelling has previously been used for health monitoring purposes, such as in the work of [28], to the author's best knowledge, it has never been used for the assessment of fault severity, as in the case of this thesis, using the assessment of the blade surface roughness in an axial turbine. Instead of being used as a design tool, the objective of meanline modelling in the fault-assessing framework is twofold: (1) to analyze the performance of the components in an existing turbine design, and (2) to serve as a means to relate the observed performance deteriorations to the physical condition of the component. To achieve these two objectives, the following challenges and limitations must be addressed during the application of the meanline model: 
(1) To represent an established turbine with a 3D blade design, the meanline representation of the flow condition must be carefully calibrated and verified, such that the performance predicted by the meanline model adequately matches that of the actual 3D design.

(2) Since the meanline design method relies on empirical correlations for loss prediction, its performance estimation is only as accurate as the empirical models employed. The "experiential" factors of the empirical models for loss components may need to be calibrated against performance data of similar blade design, in order to yield more accurate prediction. A demonstration of this point is presented in Section 3.2.

(3) At off-design conditions, the flow properties, such as Mach number, bladeloading and incidence angle, vary significantly at different locations along the blade span. Despite the development of the off-design incidence loss prediction method, such as the empirical model by Moustapha et al. [29], the dramatic variation of spanwise flow conditions may not be adequately represented by the 1D meanline model [27].

The proposed framework, presented in Chapter 4, aims to address these limitations when incorporating meanline modelling as a part of the surface roughness assessment system. Meanline modelling is versatile for implementing damage loss models, which are directly derived from the observations on damage effects through either empirical cascade testing or numerical simulation. The adaptability of damage-models and the overall 
simplicity make meanline modelling an ideal tool for analyzing the damage effects on a specific turbine under specific operating conditions.

The subsequent section presents the Jouini-Islam \& Sjolander blade roughness loss correlation system, one of the damage models developed for the AMDCKO loss system implemented in this thesis.

\subsection{Loss Correlations for Axial Turbine Blade Roughness Damage}

The surface roughness affects the flow over it in two ways: (a) it causes flow to transition from laminar to turbulent at a lower Reynolds number, and (b) it increases the turbine boundary layer skin friction coefficient. Due to the turbulent boundary layer and the enlarged surface area, surface roughness also increases the local heat transfer coefficient. A substantial amount of experimental and modelling work has been dedicated to understanding surface roughness effects on the boundary layer [30], [31], [32]. Among these works, many attempts have been made to correlate the geometric constant in the "law of the wall" to the geometric characteristics of surface roughness, such as in [33], [34] and [35], which are introduced in the following section.

\subsubsection{Equivalent Sand-Grain Roughness}

In a study of surface roughness effects on flow behaviour, sand-grain roughness is often used. Sand-grain roughness is formed by closely-packed sand grains, and was first used by Nikuradse to study the flow behaviour in roughened pipes [36]. Schlichting proposed the concept of "equivalent sand-grain roughness," which is the sand-grain 
roughness that produces the same flow resistance as the actual roughness [37]. The present thesis utilizes the correlation by Davorak \& Simpson, presented in [34], to determine the equivalent sand-grain surface roughness height $\left(k_{s}\right)$ that corresponds to a combination of the actual surface roughness height $(k)$ and the associated spacing parameter $(\lambda)$ :

$$
\frac{k_{s}}{k}=\exp \left(\frac{-5.95\left(1.103 \log _{10} \lambda-1\right)+4}{2.439}\right)
$$

The spacing parameter, which characterizes the spacing between roughness elements, is defined as follows:

$$
\lambda=\frac{\text { reference surface area }}{\text { total roughness frontal area }}
$$

In the context of an experimental study on roughened blades, the definition of the spacing parameter is the ratio of the roughened blade surface area to the total frontal area of the roughened elements [8]. Since the wind tunnel experiments on roughness were conducted for blade cascades with different spacing parameters, Schlichting's equivalent sand-grain roughness was extremely useful in relating the experimental outcomes of different roughness configurations. The concept is employed in Chapter 3 when validating a CFDbased compressibility factor against the experimental results based on a different spacing parameter configuration.

\subsubsection{Loss Correlations for Roughened Blades}

Various experimental measurements have been conducted in the past to understand the effect of roughness on blade-row performance. These works include [38], [39], and [40], which unanimously reported substantial efficiency deterioration with increased surface roughness. 
Kind et al. systematically assessed the effects of various configurations of partial roughness coverage on a low-speed planar turbine cascade, using uniformly distributed sand grains adhered to the blade surface [8]. Sand-grains with three nominal diameters were used: 338,772 and $1020 \mu \mathrm{m}$, with spacing parameters that ranged from seven to 16 (though it was near 10 for most tests). The results showed that the roughness on the suction side caused a substantially greater increase in profile loss compared to the pressure side, for the same roughness height. This was due to the higher velocity on the suction side, which in turn, caused greater entropy generation [18]. The roughness patches closer to the leading edge were found to generate greater loss increments for the same roughness height. The results also showed that the roughness had little effect on the pressure distribution around the blade and on deviation angle.

Using the experimental data collected by Kind et al. with reasonable extrapolations, Islam \& Sjolander developed the loss correlation for roughened blades [12]. The surface roughness loss was attributed to an effect on the profile loss, and the following profile loss ratio was proposed:

$$
K_{L}=\frac{Y_{P R}}{Y_{P}}=\frac{Y_{P}+\Delta Y_{R}}{Y_{p}}=f\left(k / c \times 10^{3}, \text { roughness coverage }\right)
$$

where $\Delta Y_{R}$ is the incremental loss due to roughness and $Y_{P R}$ is the profile loss including the roughness effect. $K_{L}$ is correlated with the roughness-to-chord ratio $(k / c)$. The correlations were developed for multiple configurations of roughness coverage starting from a chordwise position $(x / c)$ of $5 \%$ chord length. For design incidence, $K_{L}$ for each roughness coverage is expressed as follows: 
I. For $25 \%$ coverage of the suction surface:

$$
f(\chi)=1.0+0.058 \chi-0.0005 \chi^{2}, 0 \leq \chi \leq 25
$$

II. For $50 \%$ coverage of the suction surface:

$$
f(\chi)=1.0+0.112 \chi-0.0012 \chi^{2}, 0 \leq \chi \leq 25
$$

III. For $95 \%$ coverage of the suction surface:

$$
f(\chi)=1.0+0.151 \chi-0.0022 \chi^{2}, 0 \leq \chi \leq 25
$$

IV. For both blade surfaces covered:

$$
f(\chi)=1.0+0.192 \chi-0.0028 \chi^{2}, 0 \leq \chi \leq 25
$$

where $\chi=k_{S} / c \times 10^{3}$.

Originally, Islam \& Sjolander suggested applying $K_{L}$ directly to the profile loss corrected for compressibility and Mach number effects. Through CFD simulations using a single surface roughness configuration, Jouini et al. [10] found that the incremental roughness loss, i.e., $\Delta Y_{R}$ from Equation (2-53), decreased gradually as the Mach number increased, especially near the transonic region. The compressibility effect had a different impact on the roughness incremental loss than on the profile loss. According to this observation, Jouini et al. proposed the following correction factor to account for compressibility effects on surface roughness loss:

$$
\begin{gathered}
K_{M}=\frac{\Delta Y_{R}(M)}{\Delta Y_{R}(M=0)}=\frac{Y_{P R}(M)-Y_{P}(M)}{\Delta Y_{R}(M=0)}=\frac{a b+c M^{d}}{b+M^{d}} \\
\mathrm{a}=0.16915, \mathrm{~b}=0.55599, \mathrm{c}=1.00696, \text { and } \mathrm{d}=-8.38289
\end{gathered}
$$

where $\Delta Y_{R}(M=0)$ is the incremental surface roughness loss calculated with Equation (2-53), using the baseline profile loss without compressibility correction. $\Delta Y_{R}(M)$ is the incremental surface roughness loss at the outlet Mach number $M$. 
At the time Jouini et al. developed the CFD-based compressibility correction factor for surface roughness loss, there was not yet any empirical data available for comparison and validation. Half a decade later, Yuan [41] experimentally studied the effect of surface roughness on profile loss through transonic cascade testing, with a discharge Mach number from Mach 0.5 to 1.1. With the empirical data now available, the CFD-based correlation against the empirical data can be examined, and the applicability of the damage loss correlation system as well as the validity of the CFD-based approach by Jouini et al. can be evaluated. This is described in detail in the following chapter. 


\section{Chapter 3: Validation of the Prediction Method for Blade Roughness Damage Loss under Transonic Flow Conditions}

\subsection{Introduction}

When Jouini et al. proposed the CFD-based compressibility correction factor and the associated application method for the surface roughness loss under transonic flow conditions in 1998, there was no experimental data available for validation. Using the studies by Yuan [41], it is now possible to examine the effectiveness of the prediction method against the experimental outcomes. Since the Jouini-Islam \& Sjolander roughness loss system and the AMDCKO basic loss system are used in the proposed framework, it is also prudent to test them against the established experimental outcomes before incorporating them. Thus, the present chapter evaluates the effectiveness of the correlationbased roughness loss prediction method against the transonic experimental data obtained by Yuan, based on the HS1C transonic cascade. The chapter is organized as below.

Firstly, the loss prediction by the AMDCKO system for the HS1C transonic cascade is compared against the experimental results obtained by both Corriveau [42] and Yuan [41]. This will examine the quality of the baseline loss prediction by the AMDCKO system for a hydraulically smooth cascade blade. This also establishes a baseline profile loss prediction for subsequent evaluations of roughness loss models.

The roughness loss predictions using the correlations by Jouini-Islam \& Sjolander are then compared against Yuan's surface roughness loss measurements. The comparison is comprised of two parts: (a) the examination of the validity of the CFD-based correlation 
for $K_{M}$ against the experimental data and (b) the evaluation of the correlation-based approach in predicting losses due to various roughness configurations on the blade surface.

\subsubsection{HS1C Transonic Turbine Airfoil}

The HS1C cascade belongs to the HS1 cascade family, which was originally designed by Corriveau and Sjolander [43] to investigate the influence of loading distribution on transonic profile losses. The cascade was designed based on the mid-span turbine blade profile of an existing Pratt \& Whitney Canada (P\&WC) engine, which formed the HS1A baseline blade in the HS1 family. H1SC is an aft-loaded variant of the mid-loaded HS1A blade. Table 3-1 summarizes the blade geometry and the nomenclature of the HS1C blade following the conventions as defined in Figure 2-5.

Table 3-1: Summary of blade geometry and the design performance parameters of the HS1C cascade, adapted from Ref. [43].

\begin{tabular}{lcclcc}
\hline True Chord & $C$ & $40.4 \mathrm{~mm}$ & Design Incidence & $i_{\text {des }}$ & $-4.5^{\circ}$ \\
Axial Chord & $C_{X}$ & $37.3 \mathrm{~mm}$ & Inlet Flow Angle & $\alpha_{1}$ & $46^{\circ}$ \\
Blade Span & $h$ & $61.0 \mathrm{~mm}$ & Outlet Flow Angle & $\alpha_{2}$ & $58.5^{\circ}$ \\
Blade Pitch & $s$ & $29.14 \mathrm{~mm}$ & Inlet Mach Number & $M_{1}$ & 0.53 \\
Trailing Edge Thickness & $t$ & $1.26 \mathrm{~mm}$ & Outlet Mach Number & $M_{2}$ & 1.04 \\
Throat Opening & $o$ & $15.9 \mathrm{~mm}$ & Stagger Angle & $\zeta$ & $22.5^{\circ}$ \\
Trailing Edge Thickness- & & & & & \\
to-Throat Ratio & $t / o$ & 0.079 & Solidity & $s / C$ & 0.721 \\
Inlet Metal Angle & $\beta_{1}$ & $50.5^{\circ}$ & Aspect Ratio & $h / C$ & 1.51 \\
Outlet Metal Angle & $\beta_{2}$ & $59.0^{\circ}$ & Zweifel Coefficient & $Z$ & 0.856 \\
\hline
\end{tabular}


Using the HS1C blade, Yuan conducted experimental and numerical studies to investigate surface roughness effects on the aerodynamic performance of the HP turbine blade under compressible flow conditions. This is described in detail in the subsequent section.

\subsubsection{Source of Experimental Data}

The experimental data for the present study is from Corriveau [42] and Yuan [41]. Both sets of experimental data were obtained at the Pratt \& Whitney Canada High-Speed Wind Tunnel at Carleton University, a detailed description of which can be found in [44]. The wind tunnel is controlled by the downstream isentropic Mach number $\left(M_{2, i s e n}\right)$, which is obtained using the upstream total pressure and the downstream static pressure. This control arrangement results in the concurrent increase of Reynolds number with Mach number. The nominal measurement uncertainties associated with the cascade measurement in the wind tunnel are summarized in Table 3-2.

Table 3-2: Uncertainties of data measurement and reduction, adapted from Ref. [42].

\begin{tabular}{lcc}
\hline & Range & Uncertainty Range \\
\hline Pressure $(P)$ & 0.5 to 1.5 bar & $\pm 2 \%$ \\
Inlet Flow Angle $\left(\alpha_{1}\right)$ & $0^{\circ}$, design incidence & $\pm 1^{\circ}$ \\
Outlet Flow Angle $\left(\alpha_{2}\right)$ & $\pm 10^{\circ}$, relative to probe & $\pm 1^{\circ}$ \\
Inlet Mach Number $\left(M_{1}\right)$ & axis & \pm 0.008 \\
Outlet Mach Number $\left(M_{2}\right)$ & $<0.6$ & \pm 0.005 \\
Total Pressure Loss Coefficient $(Y)$ & $M_{2, \text { isen }}$ from 0.5 to 1.25 & \pm 0.02 to \pm 0.007 \\
Reynolds Number $(R e)$ & $M_{2, \text { isen }}$ from 0.5 to 1.25 & \pm 6000 to $\pm 9.7 \times 10^{4 \dagger}$ \\
\hline${ }_{\text {P \&WC High-Speed Wind Tunnel is designed for an experimental Reynolds number range beyond } 3 \times 10^{5} \text {, where viscous effects on the }}$ \\
profile losses are negligible.
\end{tabular}


The measurement uncertainties for Mach number and total-pressure loss coefficient increase for lower Mach numbers, due to the drift in the blowing pressure of the wind tunnel [43]. A major source of uncertainty in the Reynolds number measurement results from the decrease of flow temperature, due to the air expansion in the wind tunnel's storage tanks during each experimental run. Larger uncertainties in Reynolds number are encountered at higher outlet Mach numbers, due to the greater air expansion in the tank, and the associated higher temperature drop. Since the test section of the wind tunnel typically operates at Reynolds numbers larger than $3 \times 10^{5}$, above which the profile loss becomes a weak function of the Reynolds number, the high uncertainties have a negligible effect on the measured losses.

The HS1C performance data from Corriveau is comprised of blade loading, profile loss, outlet flow angle, and base pressure measurements at design and off-design incidences. For the purpose of the present work, the profile loss measurements at design incidence were utilized for comparison against predicted values for baseline profile loss. A selection of other measurement values, such as $M_{1}, M_{2}, R e$, and $\alpha_{2}$, were extrapolated and used as inputs for loss prediction with the AMDCKO loss system.

The measurement data by Yuan included profile loss and outlet flow angle at design incidence for both clean and roughened blades. To simulate the roughness surface due to in-service damage, round natural silica sand grains with a nominal diameter of $90 \mu \mathrm{m}$, $165 \mu \mathrm{m}$, and $225 \mu \mathrm{m}$ were used. The sand-grains were adhered to the blade surface at a spacing parameter of 20 using a dispensing system developed by Yuan. Through trial and error, the spacing parameter was controlled to be within \pm 1.5 . The test cases studied are 
summarized in Figure 3-1. The measurements for both smooth and roughened blades were made for discharge Mach numbers between 0.4 and 1.13. The corresponding Reynolds numbers were between $4 \times 10^{5}$ and $1 \times 10^{6}$, which is the range where profile loss becomes independent of Reynolds number. For the present work, the profile loss measurements for the smooth blade surface were used along with the measurements by Corriveau [42] to evaluate the baseline profile loss prediction. The roughness loss predictions were made for the roughness configurations summarized in Figure 3-1, and were compared against the measured data.

\begin{tabular}{|c|c|c|c|c|}
\hline \multirow{2}{*}{$\begin{array}{c}\text { Case } \\
\text { No. }\end{array}$} & \multicolumn{5}{|c|}{$\begin{array}{c}\text { Roughness configuration } \\
(\mathrm{k} / \mathrm{c}) \\
10^{-3}\end{array}$} & $\begin{array}{c}\text { Band start } \\
\text { location } \\
(\mathrm{x} / \mathrm{c})\end{array}$ & $\begin{array}{c}\text { Band } \\
\text { width } \\
(\Delta \mathrm{s} / \mathrm{c})\end{array}$ & $\begin{array}{c}\text { Spacing } \\
(\lambda)\end{array}$ \\
\hline 1 & 6.4 & 0.1 & 0.65 & $\sim 20$ \\
\hline 2 & 6.4 & 0.25 & 0.475 & $\sim 20$ \\
\hline 3 & 6.4 & 0.25 & 0.25 & $\sim 20$ \\
\hline 4 & 4.1 & 0.1 & 0.65 & $\sim 20$ \\
\hline 5 & 4.1 & 0.25 & 0.475 & $\sim 20$ \\
\hline 6 & 4.1 & 0.25 & 0.25 & $\sim 20$ \\
\hline 7 & 2.2 & 0.1 & 0.65 & $\sim 20$ \\
\hline 8 & 2.2 & 0.25 & 0.475 & $\sim 20$ \\
\hline 9 & 2.2 & 0.25 & 0.25 & $\sim 20$ \\
\hline 10 & & & Smooth \\
\hline
\end{tabular}

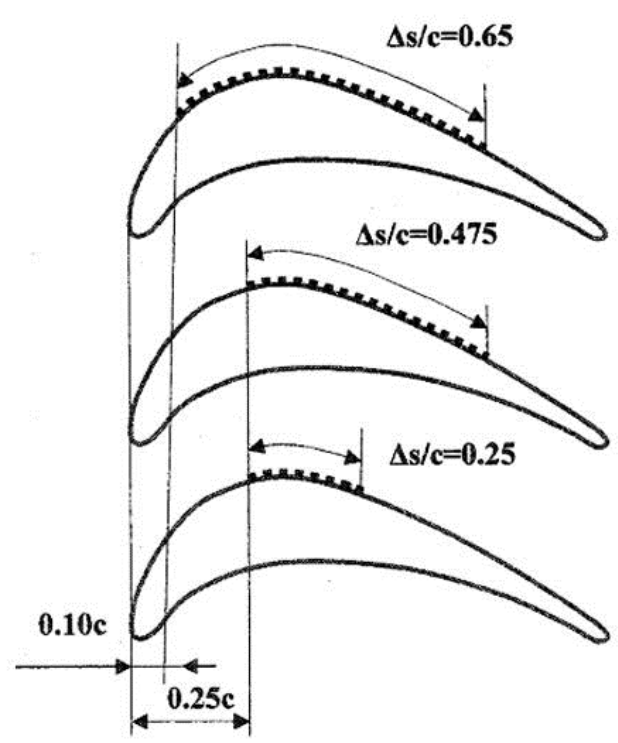

Figure 3-1: Summary of test cases and the nomenclature of roughness patch location from Yuan's study, adapted from Ref. [41]. 


\subsection{AMDCKO Profile Loss Prediction for HS1C Cascade}

A detailed description of the AMDCKO loss system is provided in Section 2.3.2. Since the AMDCKO system was used to predict the baseline profile loss in the roughness loss prediction method presented in Section 2.4.2, its prediction quality was first examined against the experimental measurements for the HS1C cascade.

Figure 3-2 shows the profile loss prediction outcome of the HS1C high-pressure transonic turbine cascade along with the measured values from both Corriveau [42] and Yuan [41]. The predicted losses included both the profile loss and the trailing edge loss components.

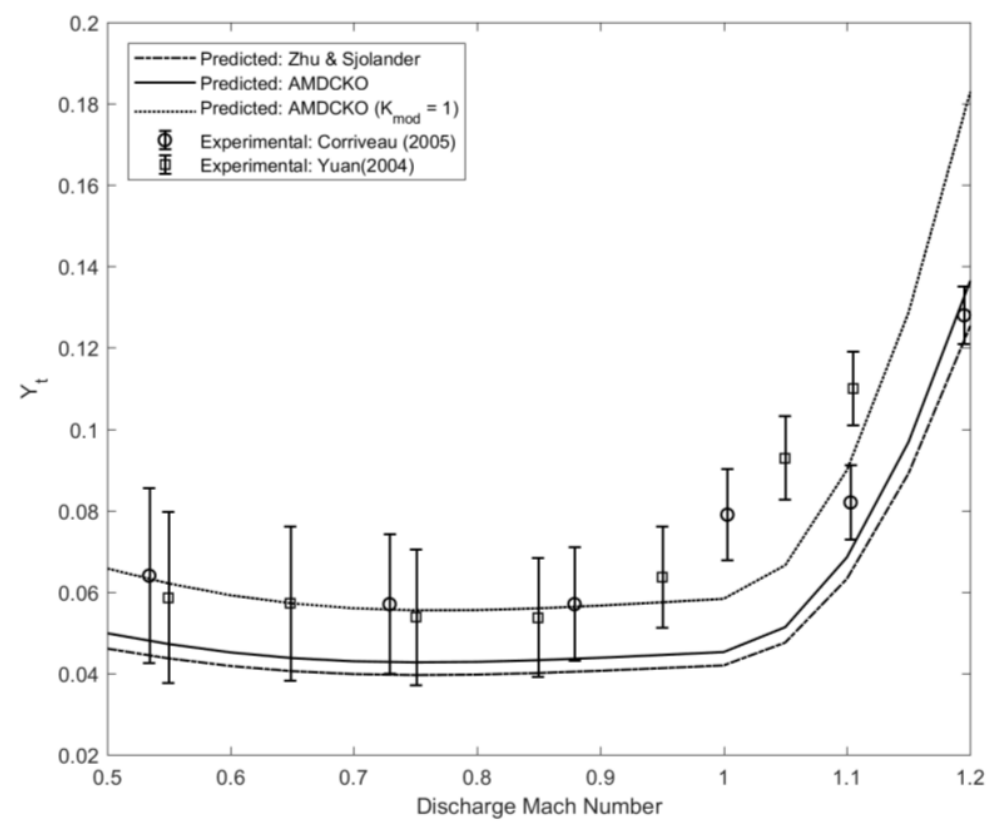

Figure 3-2: Comparison of the profile loss predictions by both AMDCKO and Zhu \& Sjolander correlations alongside the cascade tunnel measurements for the HS1C transonic turbine cascade. 
For discharge Mach numbers between 0.5 and 1.0, the predicted losses showed a moderately decreasing trend up to Mach 0.7 and then remained nearly constant, which was in agreement with the measured data by both Yuan and Corriveau. However, the predictions by both the AMDCKO correlation and the modifications by Zhu \& Sjolander were optimistic compared to the measured values. Similar underprediction by the AMDCKO system has been reported by Sooriyakumaran when applied to the transonic turbine cascades of Siemens [45]. Sooriyakumaran suggested that such underprediction may be attributed to the underestimation of trailing edge losses by the AMDCKO system. While it is hard to verify such a claim without appropriate data, another probable cause of such underprediction is the experience-based $K_{\text {mod }}$ multiplier of the profile loss, which is shown in Equation (2-33). The value of 2/3 suggested for $K_{\text {mod }}$ by Kacker \& Okapuu (or one for the Zhu \& Sjolander correlation) was based on the highly optimized turbine designs developed from state-of-art technology, and therefore, serves as a "quota" for preliminary design purposes. However, this value may not apply when analyzing an existing design, such as the HS1C cascade, for the following two reasons: (1) the blade design may not be highly optimized for minimal profile loss in the subsonic range, as the design discharge Mach number is 1.04 and (2) the performance of the cascade may not reach the expected optimal performance due to manufacturing quality and tapping installations for instrumentation purposes. Therefore, when analyzing the existing blade designs, the value of $K_{\text {mod }}$ can lie anywhere between $2 / 3$ (optimized modern design) and one (outdated design). A demonstration is shown in Figure 3-2, where the prediction using the AMDCKO correlation with $K_{\text {mod }}=1$ shows a better fit to the measurement data. 
The predicted profile loss coefficients by both AMDCKO and Zhu \& Sjolander correlations for an exit Mach number beyond unity show rapid and monotonic growth. Such growth is strongly influenced by the CFM multiplier, which unsatisfactorily simplifies the increase of the supersonic drag loss as a parabolic growth trend and neglects the complex supersonic Mach number effects. For the present case, the parabolic model underestimated the loss rise between an exit Mach number of 1 and 1.1 as compared to experimental measurements, and the prediction for Mach 1.1 lay on the edge of the error bars of the measurements. The measurements by Yuan and Corriveau differed in trend between Mach 1 and 1.1, as a "plateauing" behaviour appeared in Corriveau's observation, but not in Yuan's. The "plateauing” behaviour in supersonic loss rise has been investigated by Kibsey [46], which found base pressure to be one of the significant influencing factors. However, the understanding of other influencing factors is not yet complete, and the elaboration on this topic is beyond the scope of this thesis.

In conclusion, the predictions by the AMDCKO loss model, as well as its variant by Zhu \& Sjolander showed trend agreement with the measured data. However, the predictions from both models were well below the measured values. The $K_{\text {mod }}$ factor was calibrated to one, which yielded improved quantitative agreement between the predicted loss trend and the measured values. The empirical-based modelling presented above serves as a baseline for the evaluation of the roughness damage-loss models presented in the subsequent sections. 


\subsection{Validation of the Roughness Loss Correlations with Compressibility Effects}

\subsubsection{Validation of the Км Compressibility Correction Factor}

Section 2.4.2 provided a detailed description of the roughness damage loss correlation systems developed by Islam \& Sjolander and Jouini et al. Using a single roughness configuration over the blade surface, Jouini et al. studied the compressibility effect on roughness loss for six exit Mach number settings from Mach 0.5 to 1.3 using CFD simulation. The increments in profile loss induced by surface roughness decreased as Mach number is increased. A compressibility correction factor for surface roughness loss, i.e., $K_{M}$ from Equation (2-58), was proposed by Jouini et al. to quantify such an effect and was correlated with exit Mach number using the CFD-based data. With the experimental study on transonic roughness loss now available as a result of Yuan's work, the $K_{M}$ correlation can be tested against the experimental measurements in order to examine its validity.

$K_{M}$ values were determined for Yuan's measurements for all the roughness cases presented in Figure 3-1, using the definition in Equation (2-58). For each case, the incompressible roughness incremental loss, i.e., $\Delta Y_{R}(M=0)$, was approximated using the measured $Y_{P}$ and $Y_{P R}$ values associated with the lowest Mach number, which was 0.5 . $\Delta Y_{R}(M)$ was calculated by taking the direct difference between the measured $Y_{P R}(M)$ and $Y_{P}(M)$. Figure 3-3 compares the $K_{M}$ obtained from the experimental data with the CFDbased correlation. 


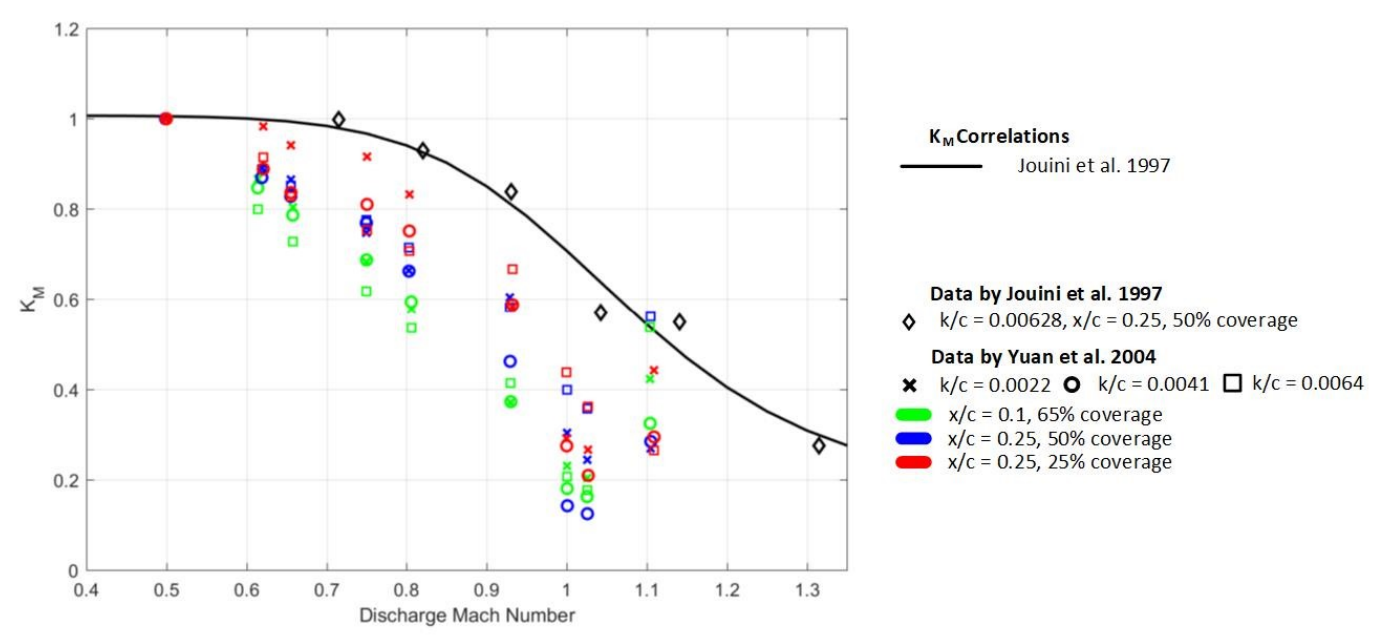

Figure 3-3: Comparison of the $K_{M}$ obtained using the experimental data of Yuan with the correlation by Jouini et al.

The comparison shows that the CFD-based correlation trend agreed with the $K_{M}$ trend obtained from experimental data between Mach 0.5 and 1, confirming the decreasing trend of $\Delta Y_{R}$ with Mach number, as observed by Jouini et al. Such a trend may be explained by the compressibility effects, which thin the boundary layer and suppress flow separation, causing a reduction in viscous losses [19]. Such a loss-reducing benefit certainly applies to the roughness loss effect. Despite trend agreement, the discrepancy in magnitude between the CFD-based predictions and empirical observations in this region was apparent. The CFD simulation underestimated the compressibility effects in the subsonic region, which was reflected by higher $K_{M}$ values. The discrepancy between the measured and predicted results in this region of outlet Mach number was also reported by Yuan et al. [9]. The shortcomings of the basic turbulence model in capturing the boundary layer phenomena 
contributed to the discrepancy, rather than the shortcomings of the surface roughness boundary-layer model employed.

The $K_{M}$ trend derived from the experimental data, as presented in Figure 3-3, suggested a significant correlation with the chordwise roughness starting position $(x / c)$ for an exit Mach number below unity. The low-speed experimental data showed that losses increased dramatically as the roughness strip was moved towards the leading edge; i.e., the lower the $x / c$ value, the higher the $\Delta Y_{R}$ due to a specific roughness height [12]. This may be caused by the extraction of a greater portion of momentum from the boundary layer by the roughness elements. The boundary layer was significantly thinner near the leading edge, as explained by Kind et al. [8]. Yuan suggested that the earlier transition from laminar to turbulent flow induced by the roughness patch may also cause substantially greater overall loss production, as a greater portion of the blade surface becomes turbulent. Therefore, the trends of $K_{M}$ in Figure 3-3 suggested that the loss-reducing benefit of the compressibility effect may be larger for blade surfaces with greater coverage of turbulent flow, caused by the earlier transition induced by the leading-edge roughness. Conversely, the $K_{M}$ trends did not suggest a strong correlation between $K_{M}$ and roughness height, partially due to the high scatter, likely caused by experimental uncertainties.

The experimentally-derived $K_{M}$ trends of different surface roughness configurations showed a rapid increase at Mach 1.1, and merged into the original $K_{M}$ trend from the correlation by Jouini et al. Such rapid jumps are likely due to the dominance of the interactions between the shock and expansion waves developed near the trailing edge, as well as the boundary-layer affected by the surface roughness patches. The merging of 
the experimentally-derived $K_{M}$ beyond an exit Mach number of 1.05 suggests that $K_{M}$ becomes a weak function of $x / c$.

In light of the observation above, a modification was made to the $K_{M}$ correlation for an exit Mach number below 1.05, with the inclusion of the starting location of the surface roughness. Figure 3-4 shows the $K_{M}$ trend of the modified correlation, which is expressed in Equation (3-1).

$$
\begin{gathered}
K_{M}=\frac{\Delta Y_{R}(M)}{\Delta Y_{R}(M=0)}=\frac{Y_{P R}(M)-Y_{P}(M)}{\Delta Y_{R}(M=0)}=\left\{\begin{array}{l}
\frac{a b+c e M^{d}}{b+e M^{d}}, M \leq 1.05 \\
\frac{a b+c M^{d}}{b+M^{d}}, M>1.05
\end{array}\right. \\
\mathrm{a}=0.16915, \mathrm{~b}=0.55599, \mathrm{c}=1.00696, \mathrm{~d}=-8.38289, \text { and } \mathrm{e}=\left(\frac{x}{c}\right)^{1.2}
\end{gathered}
$$

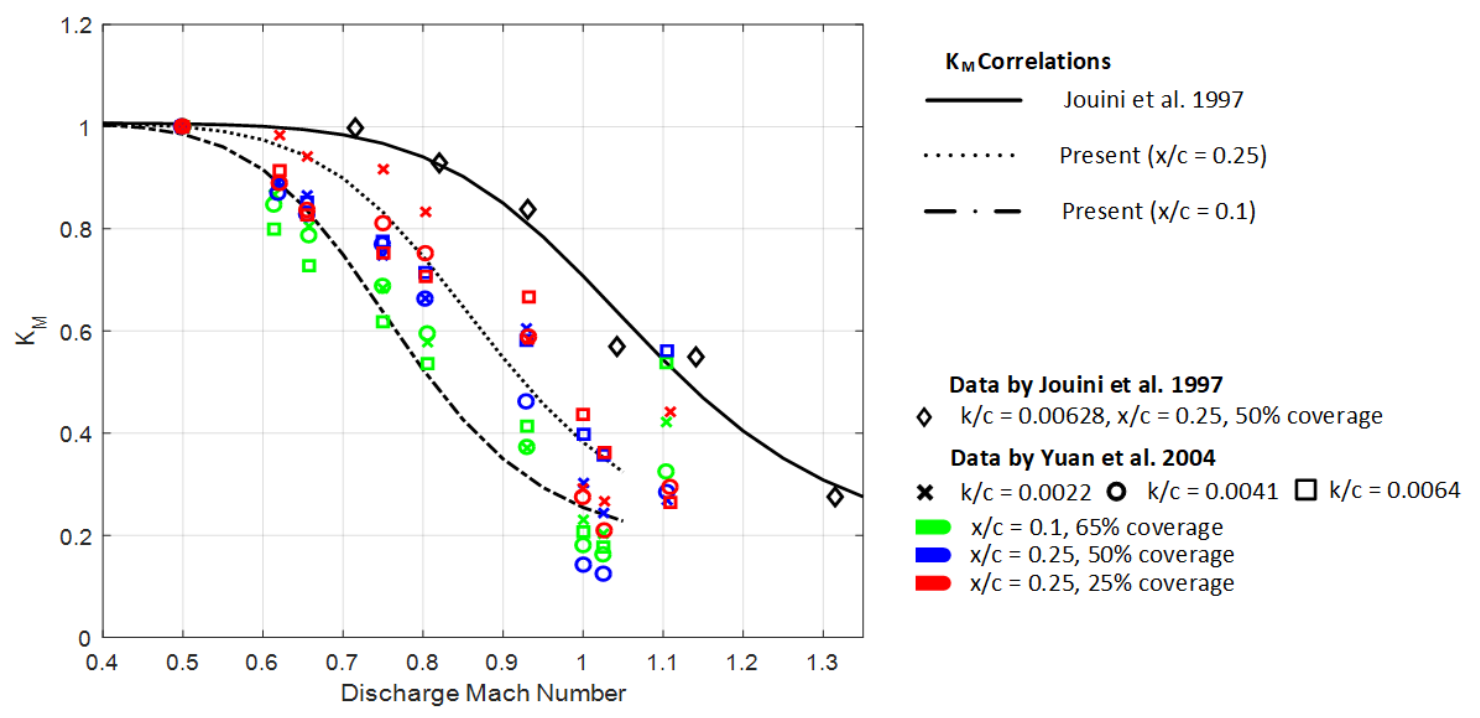

Figure 3-4: Comparison of the $K_{M}$ obtained using the experimental data of Yuan with the modified Kм correlation. 


\subsubsection{Loss Predictions for HS1C Cascade with Various Roughness Configurations}

Following the validation and modification of the compressibility correction factor for roughness loss, the present section evaluates the effectiveness of the roughness loss prediction system described in Section 2.4.2.

The correlations by Islam \& Sjolander, i.e., Equations (2-54) to (2-57), only correspond to an $x / c$ of $5 \%$ with no provision for any other $x / c$ settings. To account for the $x / c$ in the present study, a tentative correction factor was derived from the loss-toroughness position plot by Islam \& Sjolander, which is expressed as follows:

$$
\begin{gathered}
K_{x}=\frac{\Delta Y_{R}(x / c=0.05)}{\Delta Y_{R}(x / c)}=f(x / c) \\
f(x / c)=1.9683(x / c)^{3}-3.8981(x / c)^{2}+0.1047(x / c)+1.0043
\end{gathered}
$$

where $\Delta Y_{R}(x / c=0.05)$ is the incremental roughness loss corresponding to the roughness starting position at $5 \%$ of the chord. $K_{x}$ is interpolated as a third-degree polynomial, the variation of which is shown in Figure 3-5 (b).

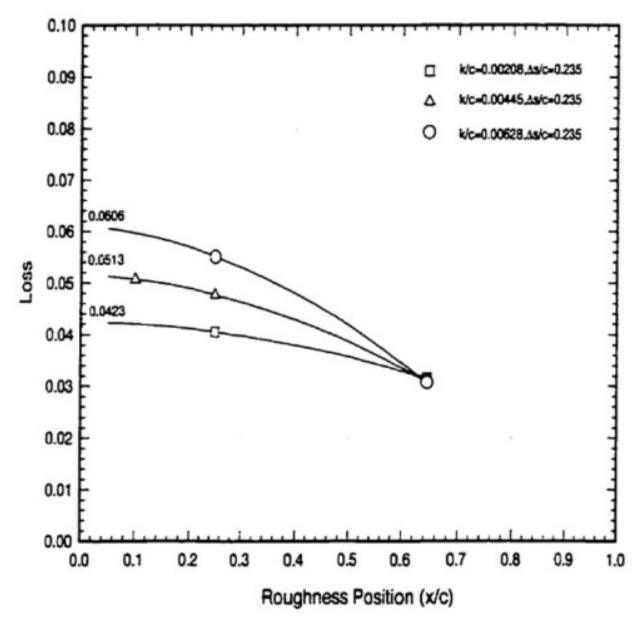

(a)

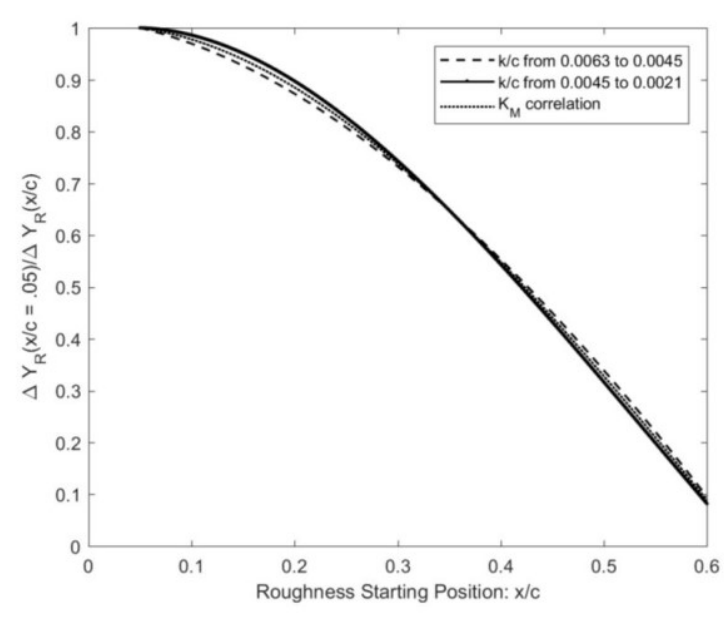

(b)

Figure 3-5: Effect of the roughness starting position (x/c): (a) variation of loss with $x / c$, adapted from Ref. [12] and (b) tentative correlation for $K_{x}$ used in the present investigation. 
It should be noted that Equation (3-2) is based on the very limited data presented in Figure 3-5 (a), and therefore only serves as a tentative correction specifically for the purpose of the investigation in the present section.

Figure 3-6 and Figure 3-7 compare the roughness loss prediction with the measurement values by Yuan for the same roughness configurations. The comparison is shown for both the total pressure loss coefficient, as well as the incremental loss induced by roughness. The experimental uncertainties are shown in the form of error bars for the incremental losses. 

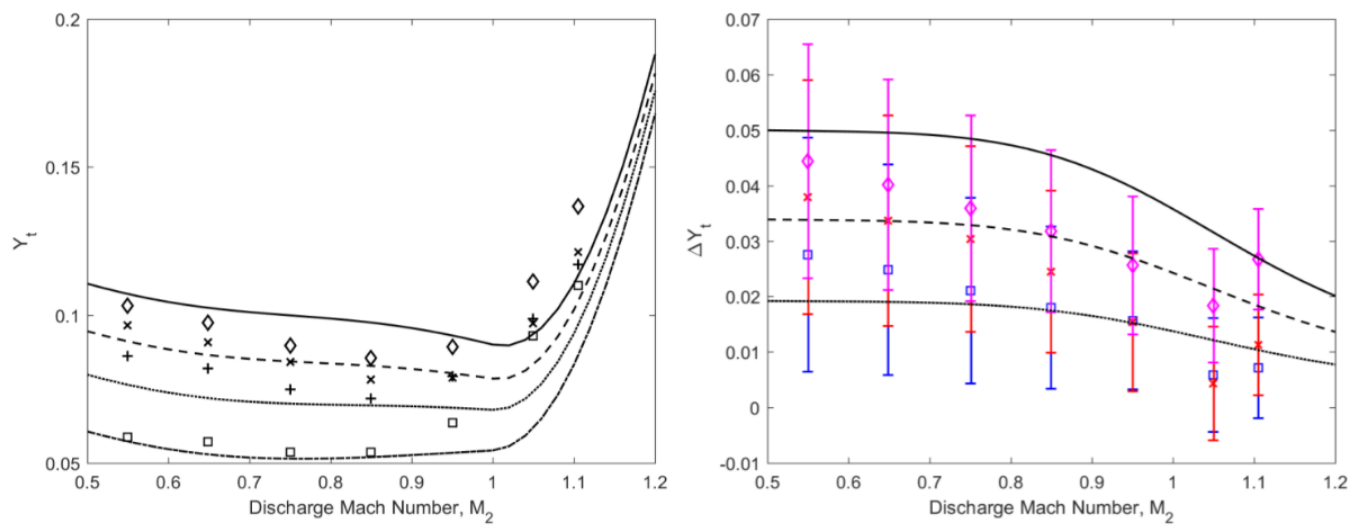

Modified $\mathrm{K}_{\mathrm{M}}$
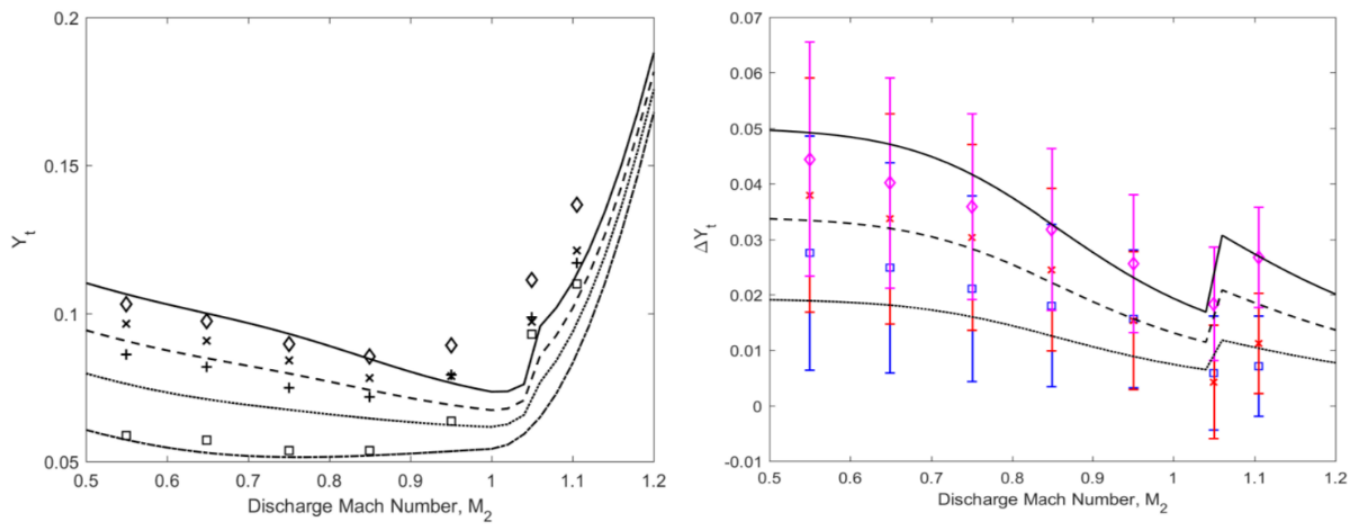

(a)

(b)

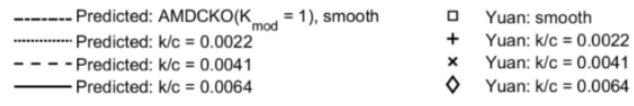

Figure 3-6: Total pressure loss coefficient with the medium roughness $b a n d(x / c=0.25, \Delta s / c=0.475)$ : (a) total pressure loss coefficient and (b) incremental loss by roughness. 
Original $\mathrm{K}_{\mathrm{M}}$
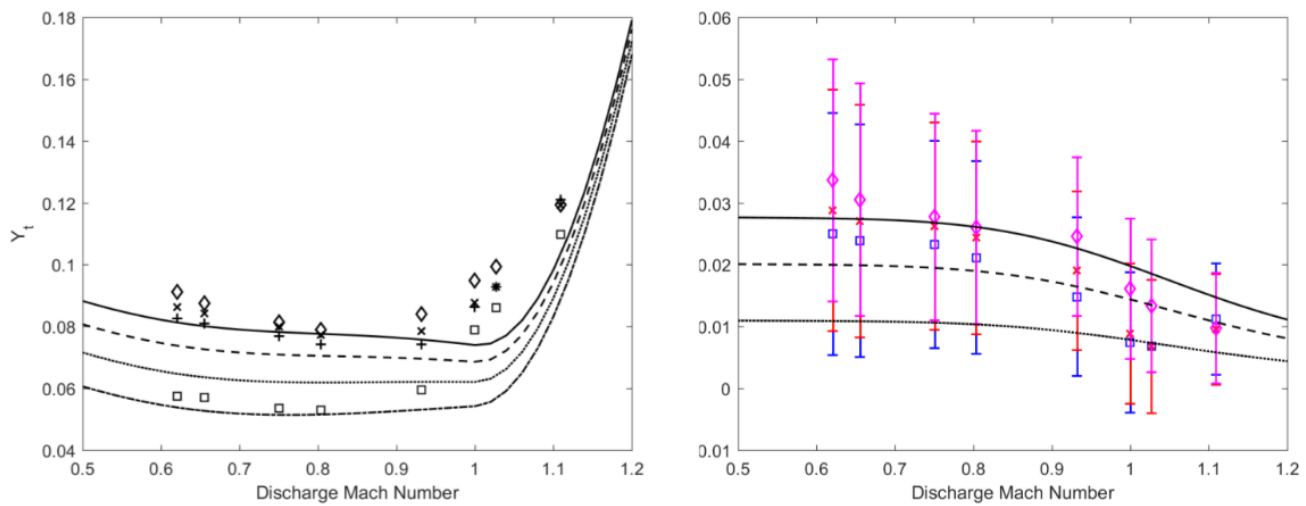

'1

Modified $\mathrm{K}_{\mathrm{M}}$

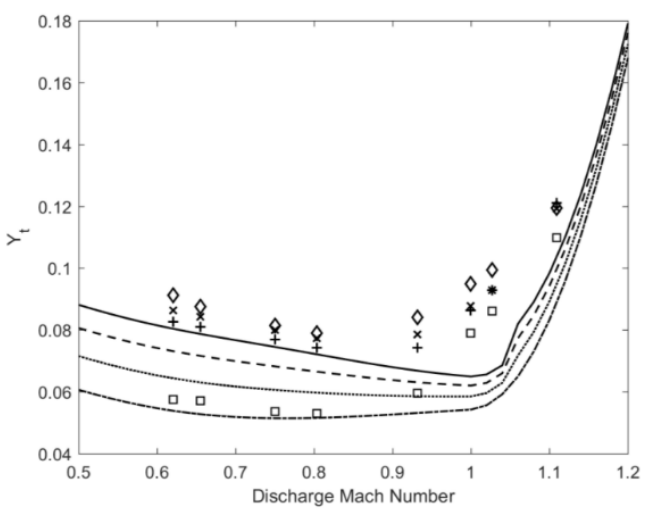

(a)

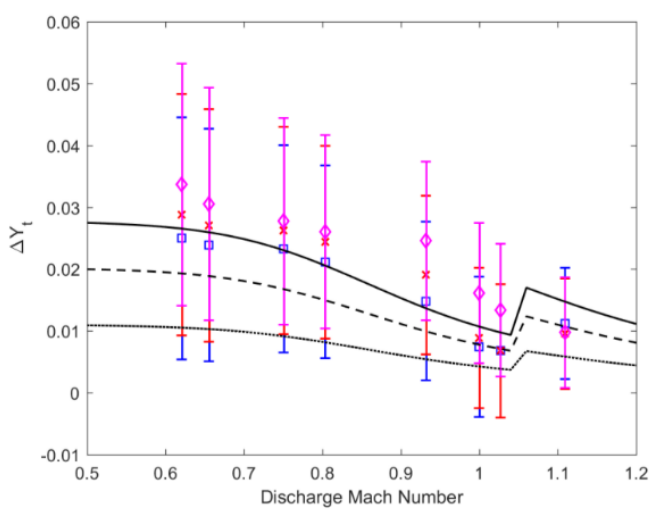

(b)

\begin{tabular}{|c|c|c|}
\hline Predicted: $A M D C K O\left(K_{\bmod }=1\right)$, smooth & $\begin{array}{l}\square \\
+\end{array}$ & $\begin{array}{l}\text { Yuan: smooth } \\
\text { Yuan: } k / c=0.0022\end{array}$ \\
\hline - - - - Predi & & Yuan: $k / c=0.0041$ \\
\hline & & Yuan: $\mathrm{k} / \mathrm{c}=0.0064$ \\
\hline
\end{tabular}

Figure 3-7: Total pressure loss coefficient with the narrow roughness band $(\mathrm{x} / \mathrm{c}=0.25, \Delta \mathrm{s} / \mathrm{c}=0.25)$ : (a) total pressure loss coefficient and (b) incremental loss by roughness. 
In general, the predicted incremental losses agreed with the experimental data, considering the experimental uncertainty margin. The predictions using the modified $K_{M}$ showed superior agreement with the measurement data over the predictions using the original $K_{M}$, and were well within the margins of experimental uncertainty for the medium rough band cases.

For the narrow band cases, the predicted incremental losses using both the original and the modified $K_{M}$ showed underestimation compared to the measurement values. This was especially significant for cases with smaller roughness heights, i.e., $k / c=0.0022$ and $k / c=0.0041$. Such discrepancies may likely be caused by the significantly large uncertainties associated with the measurement values, which were almost equivalent to the measured incremental losses in magnitude. The modelling errors associated with the Islam \& Sjolander correlations may also have contributed to the underpredictions. Since the underpredictions took place at lower Mach numbers where $K_{M}$ values are near the unity, the underpredictions were unlikely due to the correlations for the compressibility factor. Despite the underpredictions, the predicted incremental losses using both the original and the modified $K_{M}$ were still within the experimental error margins. The predictions using the modified $K_{M}$ still showed closer agreements with the trend of the measured incremental losses.

For total pressure loss predictions, those using the original $K_{M}$ showed better agreement with the measured values for an exit Mach number between 0.95 and 1.05, while the modified $K_{M}$ yielded underprediction. However, the comparison based on the total pressure loss coefficient can be deceptive, as the overprediction of the incremental loss by 
the original $K_{M}$ was compensated for by the underprediction of the baseline losses by the AMDCKO system for this Mach number range.

\subsection{Concluding Remarks on the Validation Study}

The empirical correlation-based roughness loss prediction has been evaluated against the measured data of Yuan for various roughness configurations, based on the HS1C transonic cascade. Despite the discrepancy in magnitude with the experimental data, the compressibility correction factor for roughness loss proposed by Jouini et al. was shown to be valid, and an improved variant was proposed. The predicted increment roughness loss based on a modified correction factor showed decent agreement with the measured data, despite the discrepancies in the predictions for the baseline loss of a blade with a smooth surface. This was encouraging, considering that baseline loss prediction is a major source of uncertainty in the modelling of a turbine blade. Based on the accumulated understanding of the meanline empirical-based roughness loss prediction, the following chapter presents the development of an online framework for surface roughness assessment of HP turbine blades on an in-service GTE. 


\section{Chapter 4: Online Assessment Framework for the Surface Roughness of a High-Pressure Turbine}

\subsection{Introduction}

As presented in Chapter 2, GPA and meanline analysis were originally developed for GTE condition monitoring and turbomachinery preliminary design, respectively. Each has strengths and limitations when utilized for the new purpose of component-level fault assessment. The proposed online turbine surface roughness assessment framework aims to integrate these two methods in a way that can exploit the strengths while overcoming the limitations. To achieve this goal, the online assessment framework incorporated an interfacing process to alleviate measurement uncertainties and modelling shortcomings associated with GPA. A meaningful efficiency deviation trend corresponding to the growth of surface roughness could then be extracted and passed on to the meanline analysis. The meanline turbine model in the framework was established through a system identification approach, in order to best represent the flow conditions in the modelled turbine components.

The schematic diagram of the proposed framework is shown in Figure 4-1. The HP turbine surface roughness assessment routine starts with data acquisition using the instrumentation associated with the GTE's control system, which typically acquires steadystate monitoring and operational data. The system-level GPA will then be applied to the obtained data to identify the internal degradation state of the turbine subsystem, and the associated gas path parameters (GPP), as described in Section 2.2. Through the GPA- 
meanline interfacing process, elaborated on in Section 4.3, the efficiency deviation associated with the turbine surface roughness degradation will be extracted from the GPA results. The uncertainties in the steady-state GPA will also be alleviated in this process. The extracted efficiency deviation will be passed on to the component-specific turbine meanline analysis, where the meanline aerodynamic model for the clean turbine is first identified and calibrated. The surface roughness fault severity is then determined using the received efficiency deviation. The establishment and application of the meanline surface roughness index are presented in Chapter 5.

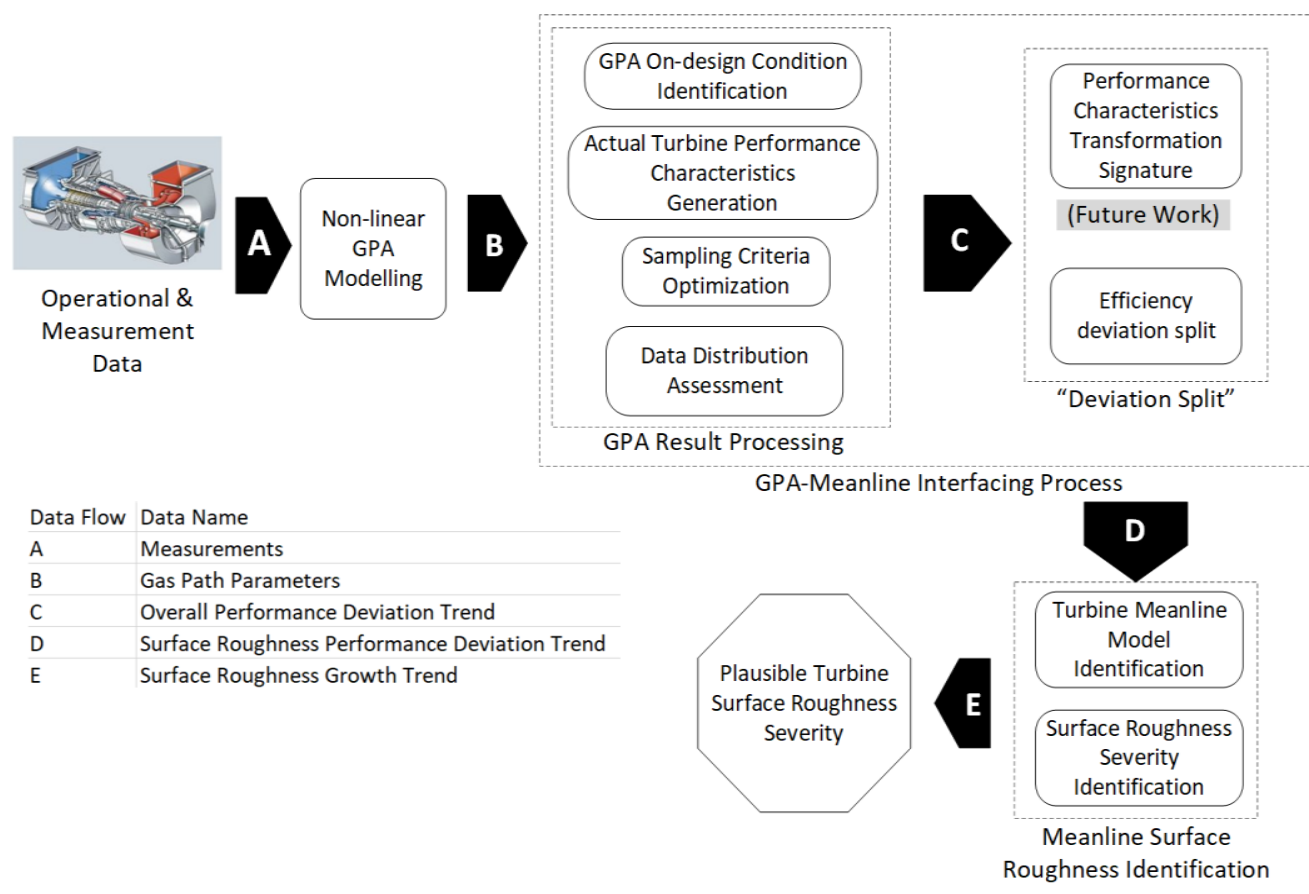

Figure 4-1: Schematic of the proposed online assessment framework for HP turbine surface roughness. 


\subsection{GPA-Meanline Interfacing Process}

The proposed GPA-meanline interfacing process samples and transforms the GPA results, i.e., GPP and $\eta$ of the turbine subsystem, into a temporal trend of efficiency deviation tailored for the meanline roughness identification process.

\subsubsection{Deviation of Performance Characteristics due to Roughness Fault}

The isentropic efficiency deviation caused by the surface roughness is determined by analysing the turbine performance characteristics' transformation, i.e. efficiency and corrected mass flow curves, which deviate from the original baseline as the turbine deteriorates. As illustrated in Figure 4-2, different types of faults in the turbine components transform the performance curves distinctively, according to MacIsaac \& Langton [47]. With the presence of a surface roughness fault, which is the main focus of the present framework, the efficiency curve shifts almost straight downward according to the observations in [40], [47], and [48]. The mass flow curve elevates marginally for a transonic turbine, due to the slight opening of the throat caused by erosion. Accordingly, the isentropic efficiency deviation due to surface roughness $\left(\Delta \eta_{t_{R}}\right)$ can be approximated by the difference between the values from the baseline efficiency curve $\left(\eta_{t_{\text {baseline }}}\right)$ and the efficiency curves established from the GPA results $\left(\eta_{t_{G P A}}\right)$ :

$$
\Delta \eta_{t_{R}}(\Omega) \approx \eta_{t_{\text {baseline }}}(\Omega)-\eta_{t_{G P A}}(\Omega)
$$

where $\Omega$ is the corrected rotor speed, which characterizes individual efficiency and mass flow curves in the turbine performance characteristics, and is defined with shaft speed $(N)$ and turbine inlet temperature (TIT) as follows: 


$$
\Omega=\frac{N}{\sqrt{T I T}}
$$
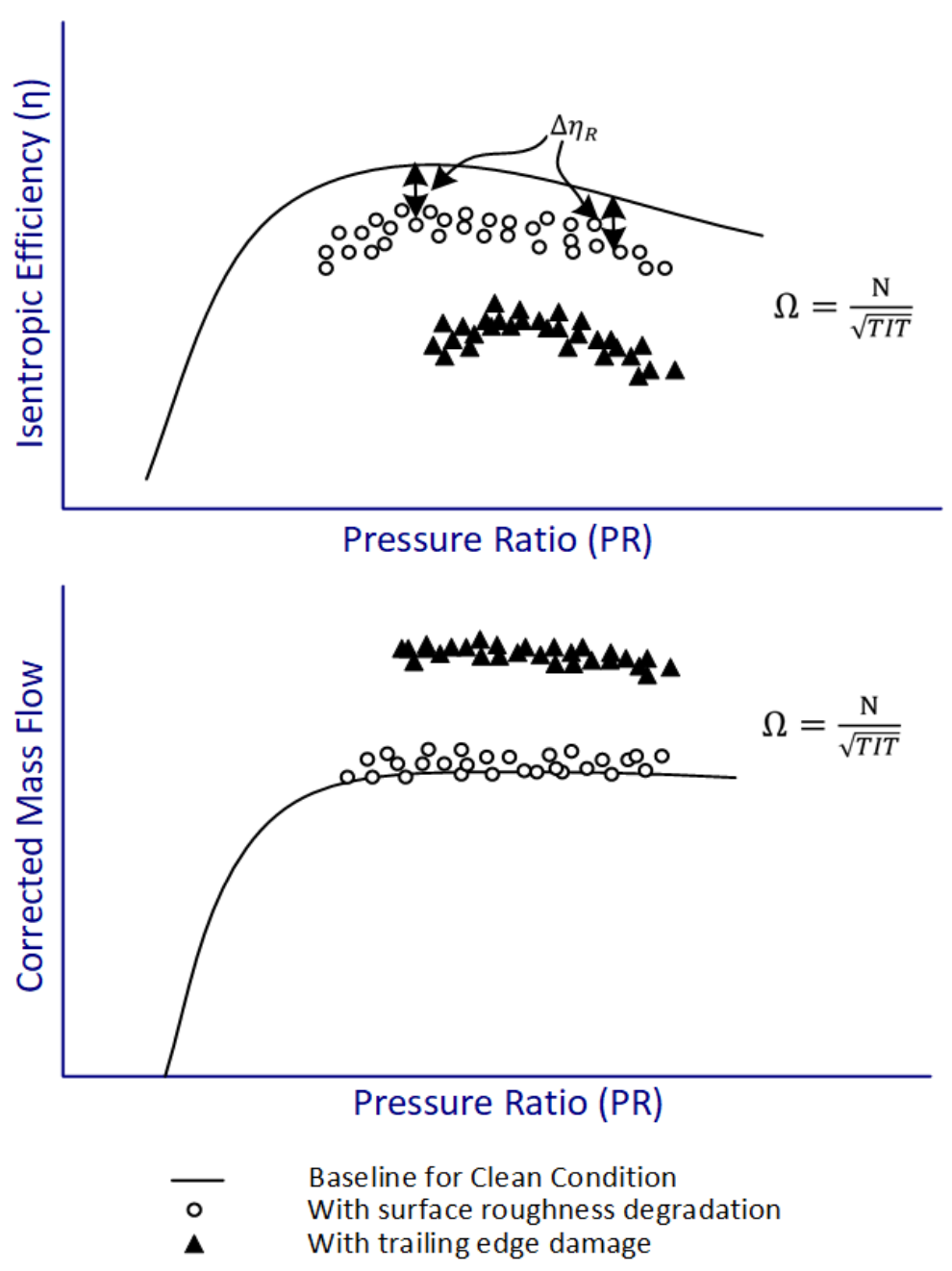

Figure 4-2: Transformation of turbine performance characteristic curves associated with the same $\boldsymbol{\Omega}$ due to different faults, reproduced based on Ref. [47].

Therefore, the end goal of the GPA-meanline interfacing process is to determine the development of $\Delta \eta_{t_{R}}$ over the operational history of the turbine for a specific $\Omega$. To achieve this objective, the interfacing process samples relevant GPA result entries and reconstructs $\eta_{t_{G P A}}$ through a series of steps. as shown in Figure 4-3. This process can be divided into two parts: (1) the identification of the design TIT and $N$ in the context of the 
GPA model, around which the GPA results will be sampled and (2) the strategic sampling process, which is optimized to address the uncertainties associated with the GPA while alleviating the fouling effect on the results.

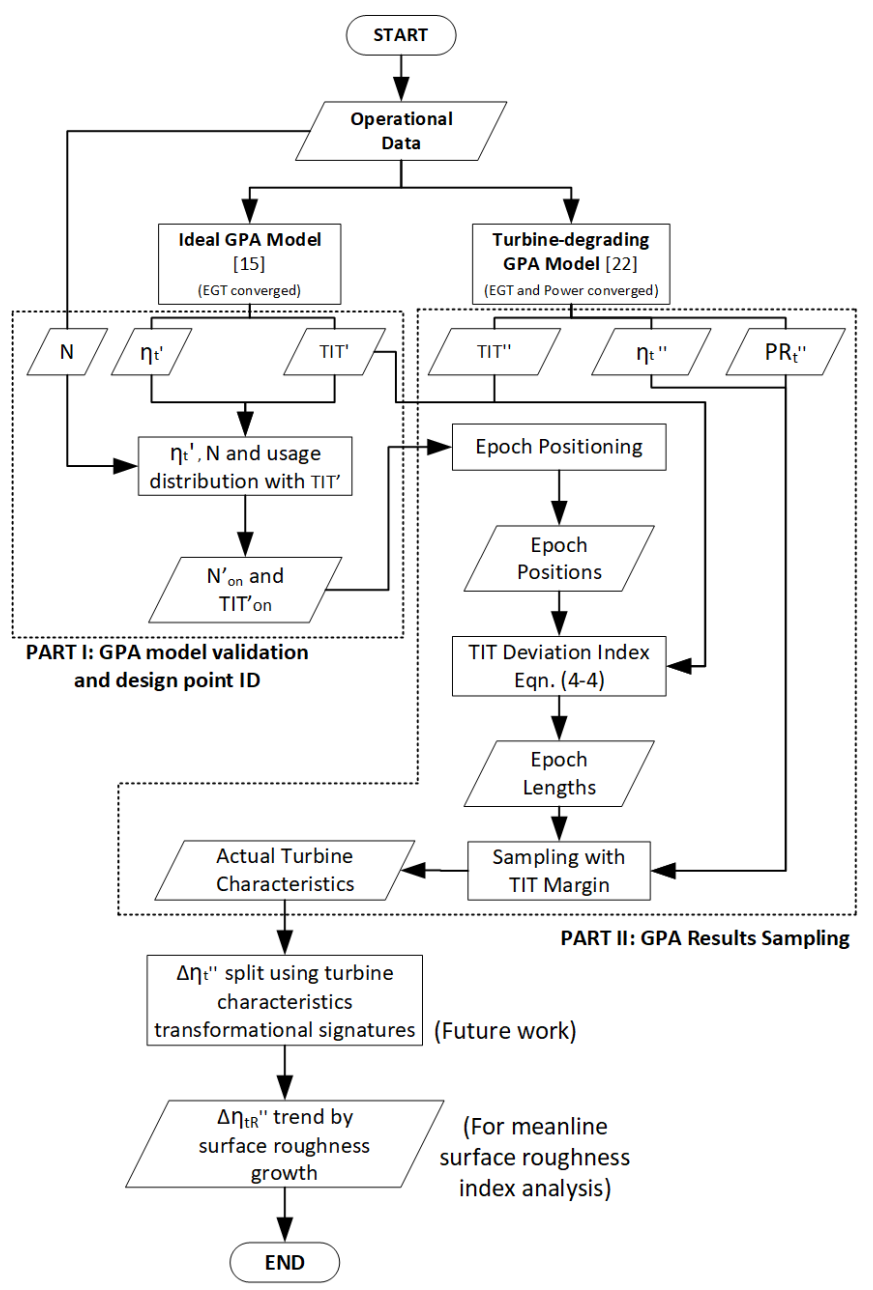

Figure 4-3: Flow chart for the GPA-meanline interfacing process.

To achieve both parts of the objective, the present interfacing process utilizes the outcomes of two GPA models, namely the ideal and the turbine-degrading models, as shown in Figure 4-2. Detailed descriptions for both models have been provided in Section 2.2 , and a brief summary is provided here for the purpose of this discussion. The ideal 
model evaluates the expected power produced by the undegraded GTE by converging only on the recorded conditions at the boundary of the GTE, such as the compressor inlet temperature and pressure, and the EGT. It was originally used to calculate the power deficit, i.e. the difference between the expected power and the recorded value from the operating system, in order to capture the compressor fouling effect. The turbine-degrading model resolves turbine long-term degradation "symptoms," i.e., deterioration of the isentropic efficiency and increase in mass flow, by scaling the turbine performance characteristics and matching the calculated power and EGT to the recorded values. Therefore, the efficiency and GPP analyzed using this model resembles the actual thermodynamic conditions in the GTE, due to the long-term degradation of turbine. Since the turbine-degrading model does not account for the compressor fouling effect, it should only be used within a limited time period after each compressor wash when compressor fouling effect is negligible. When analyzing the operating conditions affected by fouling, the model-assessed GPPs deviate from the actual conditions and should be considered physically invalid. The two models utilize the same set of thermodynamic equations as outlined in Section 2.2.1 and the same baseline component characteristic maps, only differing in algorithm. As seen in Figure 4-3, the interfacing process utilizes the results of both models according to the unique functionalities of both parts of the objective. For the convenience of discussion, the parameters associated with the ideal and the turbinedegrading model are denoted with single and double apostrophes, respectively. 


\subsubsection{Identification of Design Condition}

The first part of the interfacing process aims to identify the design $T I T, N$, and mass flow $(\dot{m})$ in the context of the GPA model, which are used to establish the sampling criterion for collecting the GPA result entries corresponding to similar operating conditions. This is because the meanline method is the most reliable when analyzing the design condition.

In the GPA analysis of a GTE with ordinary instrumentation for control purposes, $N$ is usually measured directly, whereas TIT and $\dot{m}$ are calculated through thermodynamic modelling, due to the lack of instrumentation. The TIT and $N$ in the context of the GPA model may differ from the actual case, because of inevitable modelling uncertainties, and therefore need to be identified instead of assumed as the nominal design values when used as sampling criteria. To examine the thermodynamic modelling of the GPA itself, the analysis outcome of the ideal model is used, which resolves the expected GPPs and power output based on actual ambient conditions and undegraded component characteristics. Since a GTE for electric power-generation operates at constant $N$ to match the frequency of the electric generator, the measured $N$ is expected to be constant with an error margin due to uncertainties. Accordingly, $N_{o n}^{\prime}$ can be identified by taking the average value of $N$. The identification of $T I T_{o n}^{\prime}$ and $\dot{m}_{o n}^{\prime}$ are based on the establishment of histograms of turbine efficiency $\left(\eta_{t}^{\prime}\right)$, GTE power $\left(P W_{G T E}^{\prime}\right)$, pressure ratio $\left(P R_{t}^{\prime}\right)$, and the number of available data points $\left(n^{\prime}\right)$ corresponding to different ranges of $T I T^{\prime}$. To account for the compressibility effect, corrected mass flow $\left(W_{t}^{\prime}\right)$ is used instead of $\dot{m}_{o n}^{\prime}$, which is defined as: 


$$
W_{t}^{\prime}=\frac{m^{\prime} \sqrt{T I T^{\prime}}}{P_{0_{t i}}^{\prime}}
$$

where $P_{0_{t i}}^{\prime}$ is the total pressure at the turbine inlet. The plausible values for $T I T_{o n}^{\prime}$ and $W_{t_{o n}}^{\prime}$ can then be determined based on the performance and occurrence trends in the histogram plots: (1) performance wise, $\eta_{t}^{\prime}$ and $P W_{G T E}^{\prime}$ are expected to increase as $T I T^{\prime}$ advances towards the design value, around which the turbine design is optimized, (2) for a transonic turbine, the $W_{t_{o n}}^{\prime}$ is likely a constant value as it is expected to be operated with choked flow, (3) usage wise, it is also reasonable to expect the turbine to be operated more frequently near the $T I T_{o n}$, as the plant operator will operate the GTE near its designated optimal operating point, (4) accordingly, TIT $_{\text {on }}^{\prime}$ is likely located around the peak values of the $\eta^{\prime}, P W^{\prime}$, and $n^{\prime}$ distributions. The identified $N_{o n}^{\prime}, T I T_{o n}^{\prime}$, and $W_{t_{o n}}^{\prime}$, along with the associated $P W^{\prime}{ }_{G T E}$ and $P R_{t}{ }^{\prime}$ values are compared with the nominal values as a sanity check of the GPA model and results, which need to be valid in order to be used for the surface roughness assessment.

\subsubsection{GPA Data Sampling}

With the identified $N_{o n}^{\prime}$ and $T I T_{o n}^{\prime}$, the second part of the interfacing process samples the associated results analyzed by the turbine-degrading GPA model in order to establish $\Delta \eta_{t_{R}}\left(\Omega_{o n}\right)$, while alleviating the uncertainties associated with the GPA results. The uncertainties of the GPA are mainly attributed to measurement uncertainties, GPA modelling assumptions and numerical errors. These uncertainties are exacerbated by the scantiness of the GPA results, due to the low sampling rate when acquiring the steady-state 
operational and measurement data, (as low as one log per two hours), which precludes the application of common noise filtering techniques. Consequently, to alleviate the uncertainties, the only viable option is to analyze the identical GPA results in clusters and evaluate central tendencies. The GPA results can be identical in two aspects: (1) belonging to the same short time periods where the long-term component degradations are negligible and (2) be of similar category of operating conditions, such as the design corrected speed $\left(\Omega_{\text {on }}\right)$. Based on these two aspects, the interfacing process identifies and extracts similar GPA result entries that are associated with $T I T_{o n}^{\prime}$ and $N_{o n}^{\prime}$ through "epoch" and "TIT margin," which are the sampler and the sampling criterion, respectively.

Epochs, i.e., sampling time windows, are placed at convenient times throughout the operational history of the GTE, such that the GPA results of the same period can be gathered and evaluated as a group. It is defined using two parameters: position $\left(\varepsilon_{i}\right)$ and epoch length $\left(\varepsilon_{T}\right)$, which are the starting time and the time span of the epoch, respectively. The establishment of $\varepsilon_{i}$ is dependent on the usage of the GTE. Due to the variations in ambient conditions, as well as power demands throughout a year, the operating range of an industrial GTE may vary significantly between seasons, and as a result, the turbine may be running close to $T I T_{\text {on }}$ more frequently for some periods during the year and rarely for others. Since the turbine-degrading GPA model is susceptible to compressor fouling when resolving the long-term turbine efficiency deterioration, the epochs are restricted to immediately after the compressor wash, where the compressor is considered to be in mint condition. Based on the above, $\varepsilon_{i}$ is in fact the compressor wash time after which the turbine has been running regularly near the $T I T_{o n}$. 
For each established $\varepsilon_{i}$, an appropriate $\varepsilon_{T}$ is determined such that maximum entries of the GPA result can be sampled with minimal influence of compressor fouling. Physically, the fouling effect is not manifested as the compressor efficiency deviation for a short time period after the compressor wash, until reaching a "pivoting" point after which the efficiency deterioration becomes significant, as seen in a typical case presented in Figure 5 (a). This observation indicates the existence of a time period after each compressor wash where the outcome of the turbine-degrading model is not yet affected by the fouling. Therefore, the optimal $\varepsilon_{T}$ is established at the pivoting time after which the influence of fouling on the key parameters of the turbine-degrading GPA becomes significant.

To identify the pivoting time, the effect of fouling on the turbine-degrading GPA model is examined. $T I T^{\prime \prime}$ is the parameter directly affected by compressor fouling, as discussed in Section 2.4.2; thus, a "TIT deviation index" $\left(T_{D E V}\right)$ is developed, in order to capture the pivoting point. The indicator is defined as the ratio of the TIT deviation to the expected TIT as follows:

$$
T I T_{D E V}=\left(T I T_{E x p}-T I T^{\prime \prime}\right) / T I T_{E x p}
$$

where $T I T_{E x p}$ is the expected turbine inlet temperature of an undegraded GTE under the same operational condition. Figure 5 (b) illustrates the expected trend of $T I T_{D E V}$ corresponding to the fouling effect and its intended functionality in determining the optimum $\varepsilon_{T}$. Since the ideal GPA algorithm evaluates the GPPs and the performance of the GTE in an undegraded condition, the $T I T_{\text {Exp }}$ in Equation (4-4) is essentially the $T I T^{\prime}$.

Once each epoch is established, the condition-oriented sampling criterion "TIT margin" $\left(T I T_{m}\right)$ is established to gather GPA result entries associated with $\Omega_{o n}^{\prime}$. It is 
important to distinguish between operating conditions when relating degradation to efficiency deterioration, since the aerodynamic loss varies with flow conditions, such as Mach number and incidence angle. For example, Kind et al. [8] showed that the boundary layer was more susceptible to separation by surface roughness when the airfoil was operated at off-design incidences, consequently causing greater losses compared to those at the design incidence. The sensitivity of efficiency loss to the same surface roughness severity is also influenced by compressibility effects, as seen in Chapter 3. Therefore, operating conditions must be considered when attributing efficiency deviations to fault severities. 


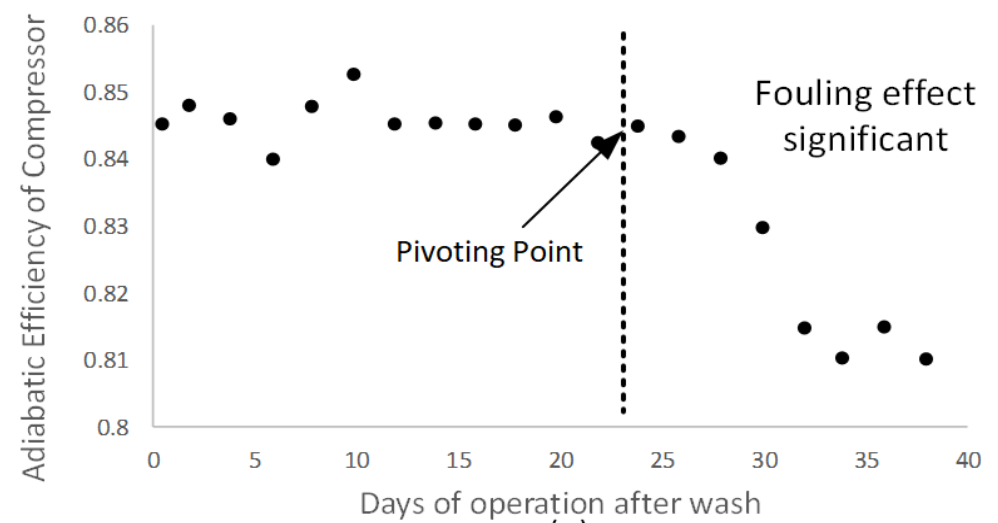

(a)

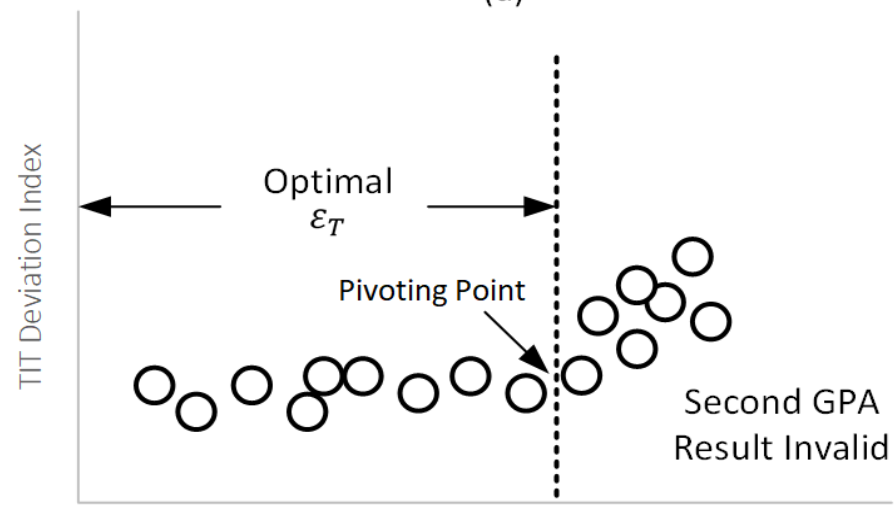

Potential Epoch length $\left(\varepsilon_{\mathrm{T}}\right)$

(b)

Figure 4-4: (a) Reported fouling effect on compressor efficiency of a typical heavy duty GTE, reproduced from Ref. [49], (b) expected trend of $\boldsymbol{T I T}_{D E V}$ corresponding to the compressor fouling effect.

It can be shown from gas dynamics that the performance of a turbine is a function of corrected rotational speed and corrected mass flow. Both $\sqrt{T I T}$ and $P_{0_{t i}}$ in Equation (4-2) and (4-3) correct for the compressibility effect. For $\Omega$, the rotational speed $N$ determines the incidence angle, i.e. the angle between the flow and the metal angle of the blade. Since the efficiency deteriorates significantly as the incidence angle deviates from its design value, $N$ is a key parameter in defining the shape of the efficiency curve. The 
curve typically remains constant during steady-state operation of a GTE for electric powergeneration purposes. Combined with the choking condition, the turbine blade is expected to always be operated at or near the design incidence, and the shape of the efficiency curve is expected to remain unchanged. With a constant $N, \sqrt{T I T}$ is the only parameter defining the operating condition. The compressibility correction in Equation (4-2) can be written in terms of a reference value:

$$
\Omega=\frac{N}{\sqrt{\theta}}=\frac{N}{\sqrt{\text { TIT } / T I T_{\text {ref }}}}
$$

Accordingly, for the present framework, the sampling criteria $T I T_{m}$ is defined around the $\sqrt{\theta}$ term in Equation (4-5) as follows:

$$
\Omega^{\prime}{ }_{o n}=\frac{N}{\sqrt{\left(T I T_{o n}^{\prime} \pm T I T_{m}\right) / T I T_{o n}^{\prime}}}
$$

The selection of $T I T_{m}$ is therefore dependent on its influence on the overall compressibility effect, which is determined by the magnitude of $T I T_{o n}$. For example, for a high $T I T_{o n}$ of $1100 \mathrm{~K}$ and a $N$ of $16500 R P M$, a $T I T_{m}$ of $10 K$ yields a $0.45 \%$ variation in the blade Mach number, which results in a negligible overall performance impact.

\subsubsection{Concluding Remarks on the Interfacing Process}

With the epochs intermittently placed throughout the operational history, results from the turbine-degrading model are sampled around the identified $\Omega_{o n}^{\prime}$, through the sampling criterion $T I T_{m}$. The $\eta_{t}$ and $P R_{t}$ of the sampled result entries for each period are then arranged into an efficiency curve, which is expected to deviate from the baseline in accordance with the degradation condition of the turbine for that period. Multiple efficiency curves corresponding to $\Omega_{o n}^{\prime}$ from different time periods can then be established, 
which in turn, reveal the temporal trajectory of the curve transformation from the baseline, as illustrated in Figure 4-5. The temporal trend of $\Delta \eta_{t_{R}}\left(\Omega^{\prime}{ }_{\text {on }}\right)$ can then be established using the approximation presented in Equation (4-1).

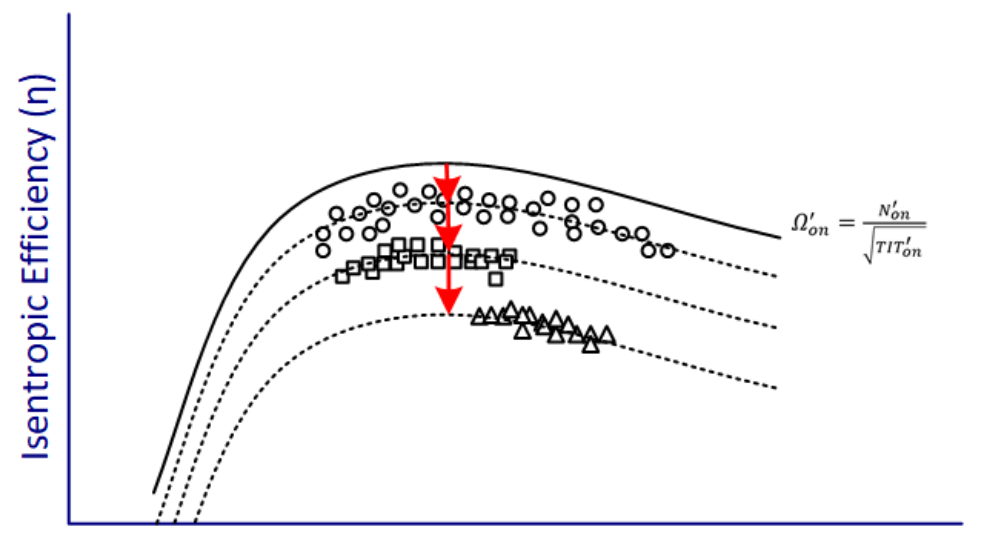

Pressure Ratio (PR)

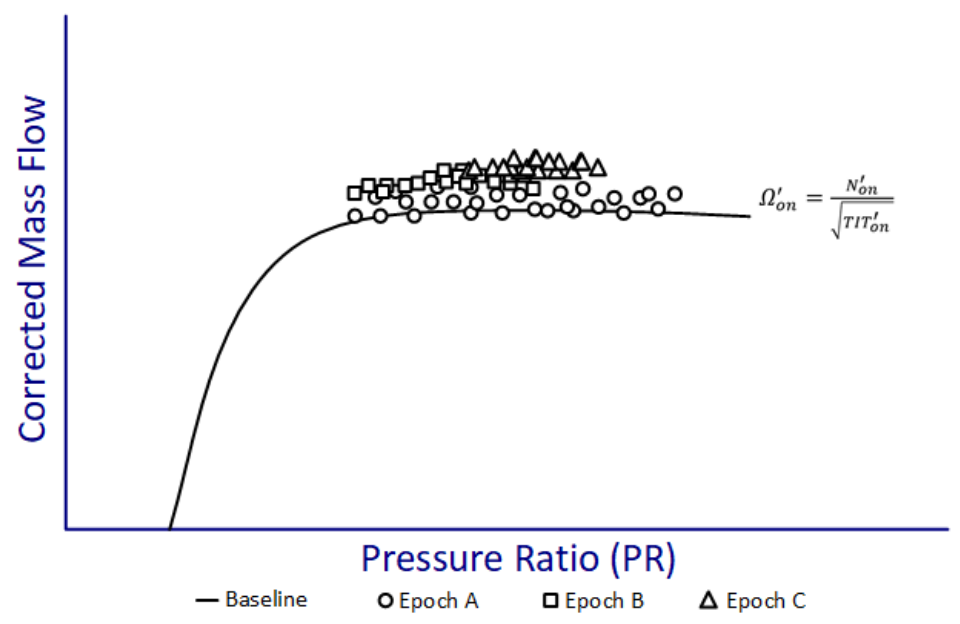

Figure 4-5: Efficiency curves corresponding to same $\Omega_{o n}^{\prime}$ from different epochs reveal the temporal trajectory of the curve transformation due to blade roughening.

It is important to note that, due to the lack of data in the open literature on the efficiency curve transformation trajectory in relation to fault types other than surface roughness, the $\Delta \eta_{t_{R}}$ approximation method in Equation (4-1) could only be applied to industrial GTEs. In these cases, surface roughness was the predominant cause of 
degradation, due to rugged blade design as well as less frequent and longer transient operations. Nevertheless, the transformational signature-based component-level fault feature extraction will be explored in the author's future work, so as to extend the present framework's ability to the assessment of multiple fault type severities. Examples include tip-clearance enlargement and trailing edge damage, which are more likely to occur in aeroengines and emergency power units.

\subsection{Meanline Roughness Identification Process}

The meanline roughness identification process was developed to resolve the surface roughness severities from the extracted efficiency deviations. Different from the designoriented meanline analysis procedure, the proposed identification process first established the mean-radii representations of the flow conditions across the span of the existing undegraded turbine. It utilized the nominal performance values and key design parameters, in order to assess the component losses associated with the original design. Based on the established meanline representative turbine model, the second part of the identification process matched the surface roughness severity to the $\Delta \eta_{t_{R}}$ obtained from the GPAmeanline interfacing process, which in turn, resolved the surface roughness growth trend. The details of the two-part identification process are shown in Figure 4-6. 


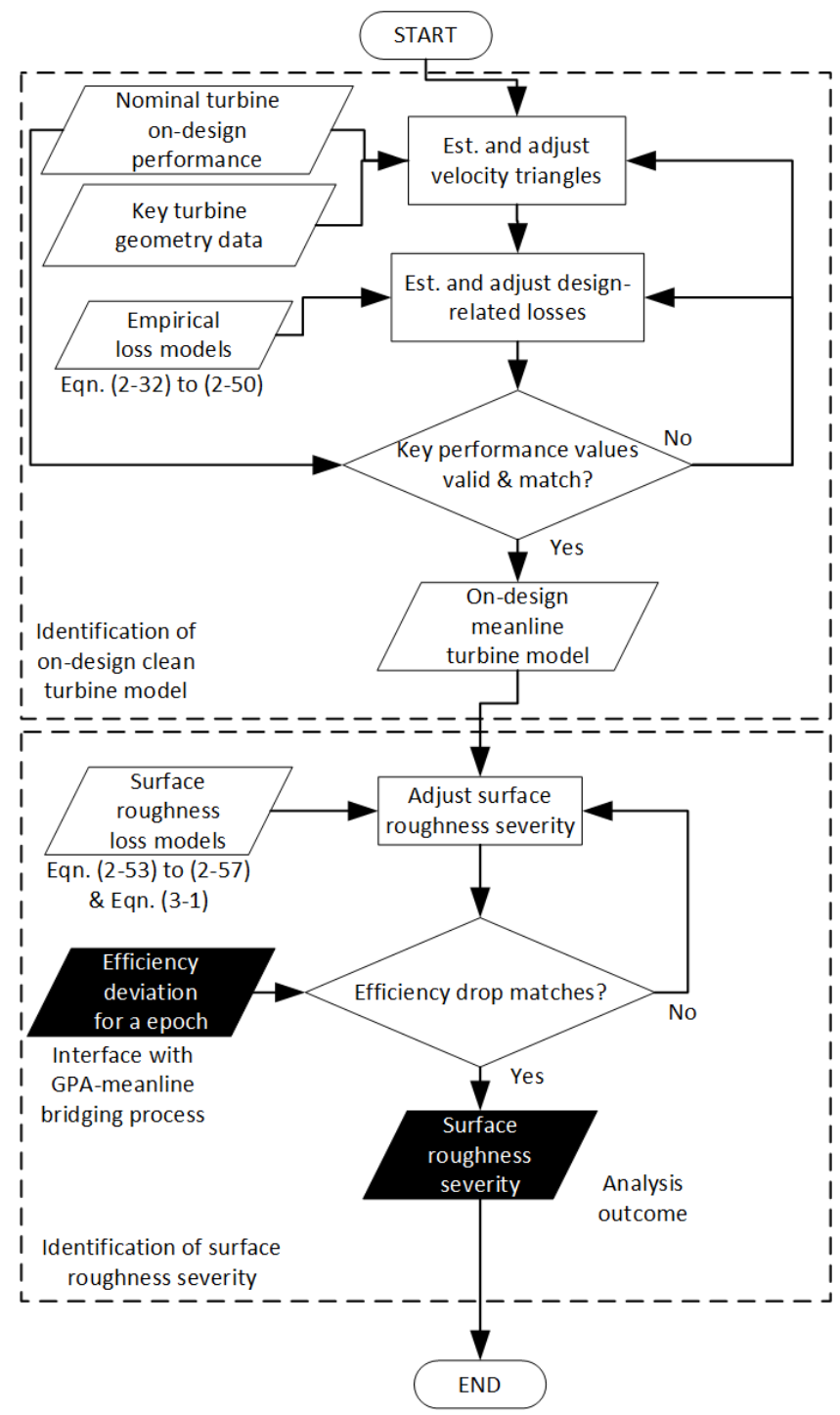

Figure 4-6: Flow chart of the proposed meanline surface roughness fault matching process.

The first part of the identification process aims to establish the meanline representation of the flow conditions across the span of the HP turbine vanes and blades, such that the basic performance parameters of the model match the nominal values of the actual turbine. Although the availability of information on a particular turbine component varies, the minimum information required to establish the meanline model for an existing 
turbine design includes: (1) annuli gas path geometry, (2) basic blade geometry specifications, such as chord length, blade angles, stagger angle, solidity, and the maximum thickness-to-chord ratio, and (3) turbine inlet flow conditions, including flow angle, total pressure and temperature, in order to establish the velocity triangles, and consequently resolve the energy transfer by the turbine as well as the aerodynamic losses. To ensure that the meanline simplification model represents the actual turbine, key performance parameters, such as pressure ratio $\left(P R_{\text {stage }}\right)$, work coefficient $\left(\Psi_{\text {stage }}\right)$, flow coefficient $\left(\phi_{\text {stage }}\right)$, reaction $\left(\Lambda_{\text {stage }}\right)$, and the isentropic total-to-total efficiency $\left(\eta_{\text {stage }}\right)$ are also used as modelling constraints. The definitions of these parameters are presented in Section 2.3.1.

Since the surface roughness loss correlation model is a function of profile loss, as shown in Equation (2-53), and the profile loss correlation of the AMDCKO system is correlated by relative flow angles [19], the focus of the clean turbine meanline modelling should be on establishing accurate relative flow angles across the span. The profile loss assessment can also be improved by adjusting experience-based correction factors, such as the $K_{\text {mod }}$ in Equation (2-33). This approach has been demonstrated and evaluated in Chapter 3.

Depending on the complexity of the turbine design, as well as the availability of information on the design, iterative parameter adjustment varies for different cases and requires engineering judgement.

The second part of the identification process resolved the surface roughness severity by matching the damage correlation-incorporated meanline model to the efficiency deviations, i.e., $\Delta \eta_{t_{R}}\left(\Omega_{o n}^{\prime}\right)$ obtained from the GPA-meanline interfacing process. The 
surface roughness loss prediction system presented in Section 2.4 was incorporated into the previously identified meanline model for the clean turbine. Since the surface roughness loss had a minimal effect on the outlet flow angles, according to the empirical observation of Kind et al. [8] and Yuan et al. [9], the velocity triangle deviation angles of the established meanline model were held constant.

\subsubsection{A Proof-of-Concept Index: The Quarter Surface Coverage Index}

As a proof-of-concept, with the objective low-fidelity assessment of surface roughness condition, a surface-roughness-level index was created for the present work. The index was defined as the surface roughness height at $25 \%$ coverage starting at the $5 \%$ chord position from the leading edge. Since the surface roughness on the suction side of the airfoil has a significantly higher influence on the aerodynamic loss, according to the observation of Kind et al. [8], the index was defined using the roughness level on the suction surface. The selection of $25 \%$ coverage was in accordance with the observations of the first stage HP turbine blades of two different aeroengines by Taylor [6], which revealed the leadingedge area as the region with distinctively higher surface roughness severity compared to the rest of the suction surface. The index also assumed the same surface roughness height for vanes and blades. The identification process iteratively resolved the meanline surface roughness index by matching the efficiency drop predicted by the meanline model to the trend in $\Delta \eta_{t_{R}}\left(\Omega_{o n}^{\prime}\right)$, which was determined through the GPA-meanline interfacing process. 
In the subsequent chapter, the proposed online surface roughness assessment framework was applied to the three-year operational data of an industrial GTE in order to evaluate the feasibility of the framework. 


\section{Chapter 5: Framework Implementation and Discussion}

\subsection{Introduction}

The effectiveness of the proposed online turbine surface roughness assessment framework was examined through its application to the three-year operational data of a 4 MWe Siemens/Ruston SGT-100 “Typhoon” cogeneration GTE powerplant. This particular engine was selected as the subject of the study, due to the availability of a three-year operational $\log$, which was presented in Section 1.3 , as well as the extensive documentation of the engine and its turbine design in the open literature. The present study aims to assess the surface roughness level of the turbine blade through the developed framework using the logged measurement data from the operating system of the GTE.

Due to a lack of observation data associated with this particular engine, the present validation was achieved using the available measurement values reported by Tarada [7], which included the observation of several Ruston-manufactured HP turbine vanes and blades made of the IN939 alloy.

\subsection{Ruston/Siemens SGT-100 Typhoon GTE}

The nominal design values of the original introductory model of this engine are summarized in Table 5-1. The original turbine vanes and blades were manufactured with the IN939 alloy [50]. It should be noted that the Typhoon GTE series has been subjected to multiple design upgrades since its introduction. For example, the turbine inlet 
temperature has been reportedly raised to $1100^{\circ} \mathrm{C}$, which consequently increased power output, mass flow rate, pressure ratio, exhaust gas temperature, and thermal efficiency [51].

Table 5-1: Basic performance of the original Ruston Typhoon GTE.

\begin{tabular}{lcc}
\hline Power & 4100 & $\mathrm{~kW}$ \\
Mass Flow & 17.1 & $\mathrm{~kg} / \mathrm{s}$ \\
Pressure Ratio & 12.81 & - \\
Turbine Inlet Temperature & 1053 & ${ }^{\circ} \mathrm{C}$ \\
Exhaust Gas Temperature & 500 & ${ }^{\circ} \mathrm{C}$ \\
Turbine Shaft Speed & 16570 & $\mathrm{RPM}$ \\
Thermal Efficiency & $32 \%$ & - \\
\hline
\end{tabular}

The geometry and performance of the HP turbine were obtained from Hannis et al. [52]. The meanline modelling used the annuli geometry as well as the measured performance of the turbine stage, which are presented in Figure 5-1 and Table 5-2, respectively.

Table 5-2: Nominal performance for the HP turbine of the Typhoon GTE.

\begin{tabular}{lcc}
\hline Inlet Pressure & 130 & $\mathrm{kPa}$ \\
Inlet Temperature & 1075 & ${ }^{\circ} \mathrm{C}$ \\
Specific Heat Ratio & 1.31 & - \\
Gas Constant & 287.0 & $\mathrm{~J} / \mathrm{kgK}$ \\
Mass Flow & 17.59 & $\mathrm{~kg} / \mathrm{s}$ \\
Turbine Shaft Speed & 17384 & $\mathrm{RPM}$ \\
Pressure Ratio & 3.9 & - \\
Mean Dia. Flow Coef. & 0.57 & - \\
Mean Dia. Work Coef. & 2.0 & - \\
Mean Dia. Reaction & 0.45 & - \\
Isentropic Stage Efficiency & $85.5 \%$ & - \\
\hline
\end{tabular}




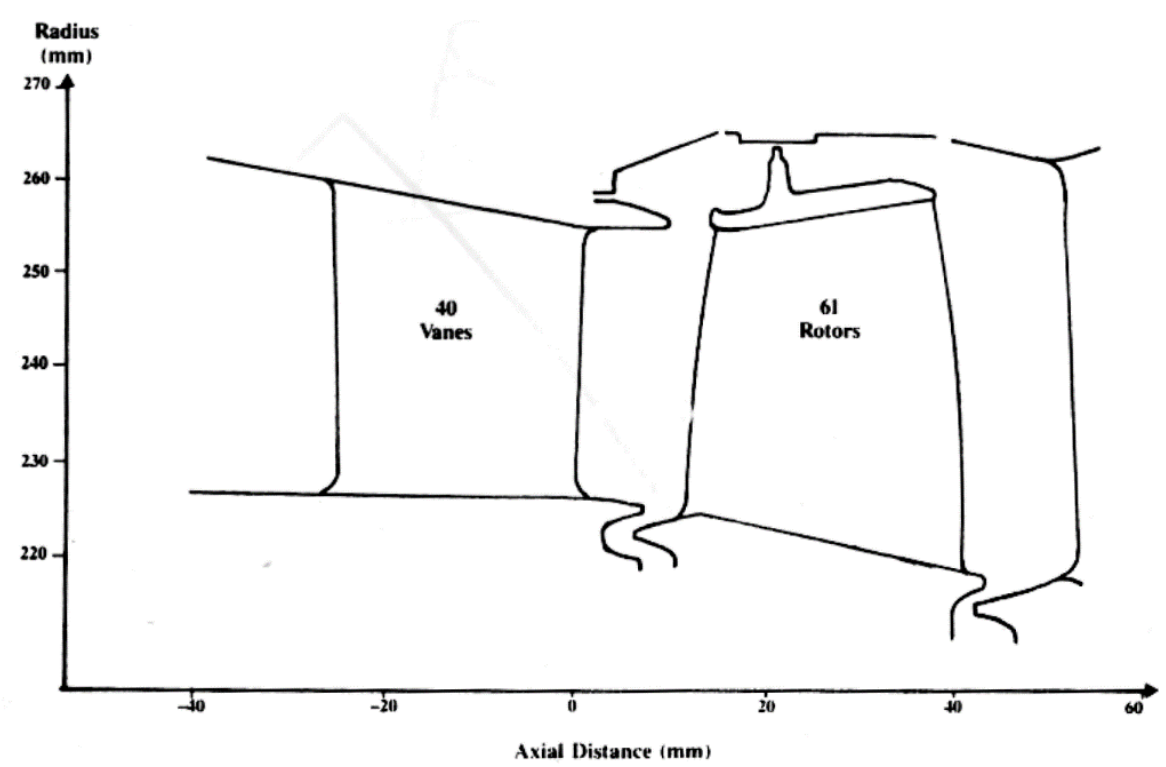

Figure 5-1: Flow path geometry of the HP turbine stage, adapted from [52].

\subsection{GPA-Meanline Interfacing Process}

The results of the GPA for both the ideal and the turbine-degrading models are presented in Section 2.2.4. As a co-generation GTE, the value of $N$ throughout its operational history was relatively constant, as seen in Figure 1-3. Closer examination of this vector shows that the value of $N$ ranged from 16411 to 16519 , with a mean and median of 16458 . Based on this observation, the on-design $N^{\prime}$ was considered to be constant at 16458, and as a result, the variation of $T I T^{\prime}$ predominantly effected the value of $\Omega$. Therefore, the performance curves of the turbine in the present GTE were characterized by the variation in the compressibility effect.

Figure 5-2 shows the histogram of various performance parameters and occurrences with $T I T^{\prime}$; i.e., the value of TIT from the ideal GPA. Based on this observation, two 
candidate values for $T I T_{\text {on }}^{\prime}$ were identified based on the "peak efficiency" and the "maximum occurrence" criteria specified in Section 4.2.2, which were $1090^{\circ} \mathrm{C}$ and $1095^{\circ} \mathrm{C}$, respectively. Due to a lack of information on the "Typhoon" variant studied, there were no specific comparable nominal values apart from those in the literature. Nevertheless, Figure 5-2 shows that the identified values were within reasonable range of the reported $T I T_{\text {on }}$ of the improved model, i.e., $1100^{\circ} \mathrm{C}$, and the associated performance parameters were sufficiently close to the nominal values summarized in Table $5-1$. This demonstrates the validity of the thermodynamic model used in the GPA. The corrected mass flow, $W_{t}^{\prime}$, was a constant line, indicating the choking of the turbine for all ranges of $T I T^{\prime}$ 

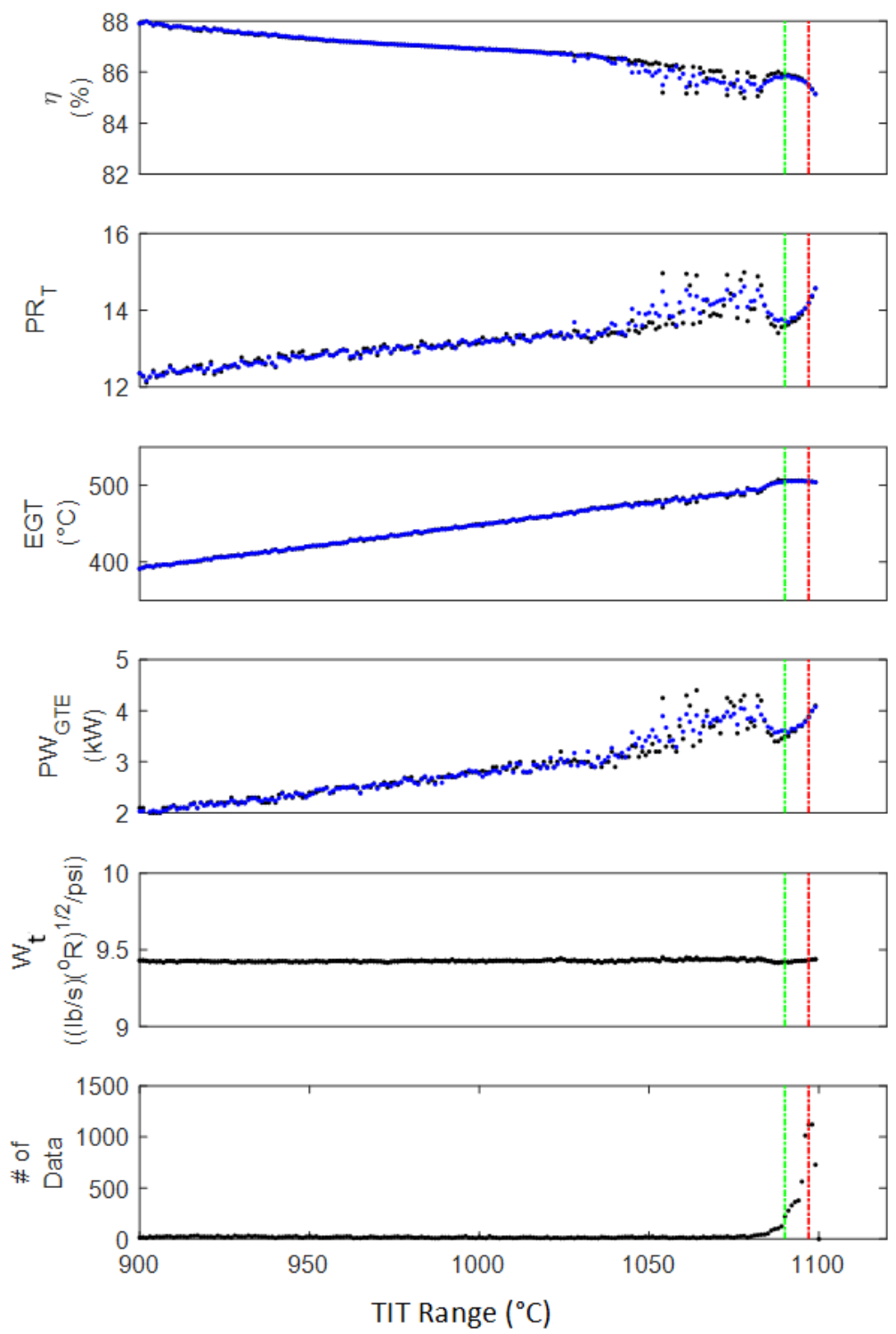

- Mean --- Choice 1, 1090 (Highest Efficiency)

- Median $\quad$--- Choice 2, 1095 (Highest \# of Data)

Figure 5-2: Distribution of key performance parameters with various TIT ranges.

Since both $N^{\prime}$ and $W_{T}$ were relatively constant, the incidence angle of the rotor, which significantly affects the losses, was also considered constant. Around the baseline $\operatorname{TIT}_{\text {on }}^{\prime}$ value of $1090^{\circ} \mathrm{C}$, a $\operatorname{TIT}_{m}$ of $10^{\circ} \mathrm{C}$ was selected, which led to a $\operatorname{TIT}^{\prime}$ sampling range 
between $1080^{\circ} \mathrm{C}$ and $1100^{\circ} \mathrm{C}$, corresponding to $\pm 0.37 \%$ variation of the compressibility correction term $\sqrt{T I T}$.

\subsubsection{Epoch Establishment}

Figure 5-3 shows the epoch placement throughout the operational history. The establishment of $\varepsilon_{i}$ was based on the available data corresponding to the defined $T I T^{\prime}$ range near each wash, which can be clearly visualized in Figure 5-3 (b). Table 5-3 summarizes the detailed $\varepsilon_{i}$ establishment for each epoch. The GTE rarely operated near the identified TIT $_{\text {on }}$ in the summer season, due to the lack of demand for electric power and heat.

Table 5-3: Epoch placement and corresponding time in the operational history.

\begin{tabular}{|c|c|c|c|c|}
\hline \multicolumn{5}{|c|}{$T_{I T}^{\prime}: 1080-1100^{\circ} \mathrm{C}$} \\
\hline & $\begin{array}{l}\text { Colour } \\
\text { Code }\end{array}$ & Placement $\left(\varepsilon_{i}\right)$ & Correspond. Time & Length $\left(\varepsilon_{T}\right)$ \\
\hline$\varepsilon_{1}$ & Yellow & Day 1 & Late March, 2008 & 12 days \\
\hline$\varepsilon_{2}$ & Green & Day 231 & Mid Nov., 2008 & 8 days \\
\hline$\varepsilon_{3}$ & Cyan & Day 356 & Late March, 2009 & 4 days \\
\hline$\varepsilon_{4}$ & Blue & Day 610 & Late Nov., 2010 & 24 days \\
\hline$\varepsilon_{5}$ & & Day 734 & Early April, 2010 & 23 days \\
\hline$\varepsilon_{6}$ & Magenta & Day 930 & Mid Oct., 2010 & 18 days \\
\hline$\varepsilon_{7}$ & Black & Day 1026 & Late Jan., 2011 & 15 days \\
\hline
\end{tabular}



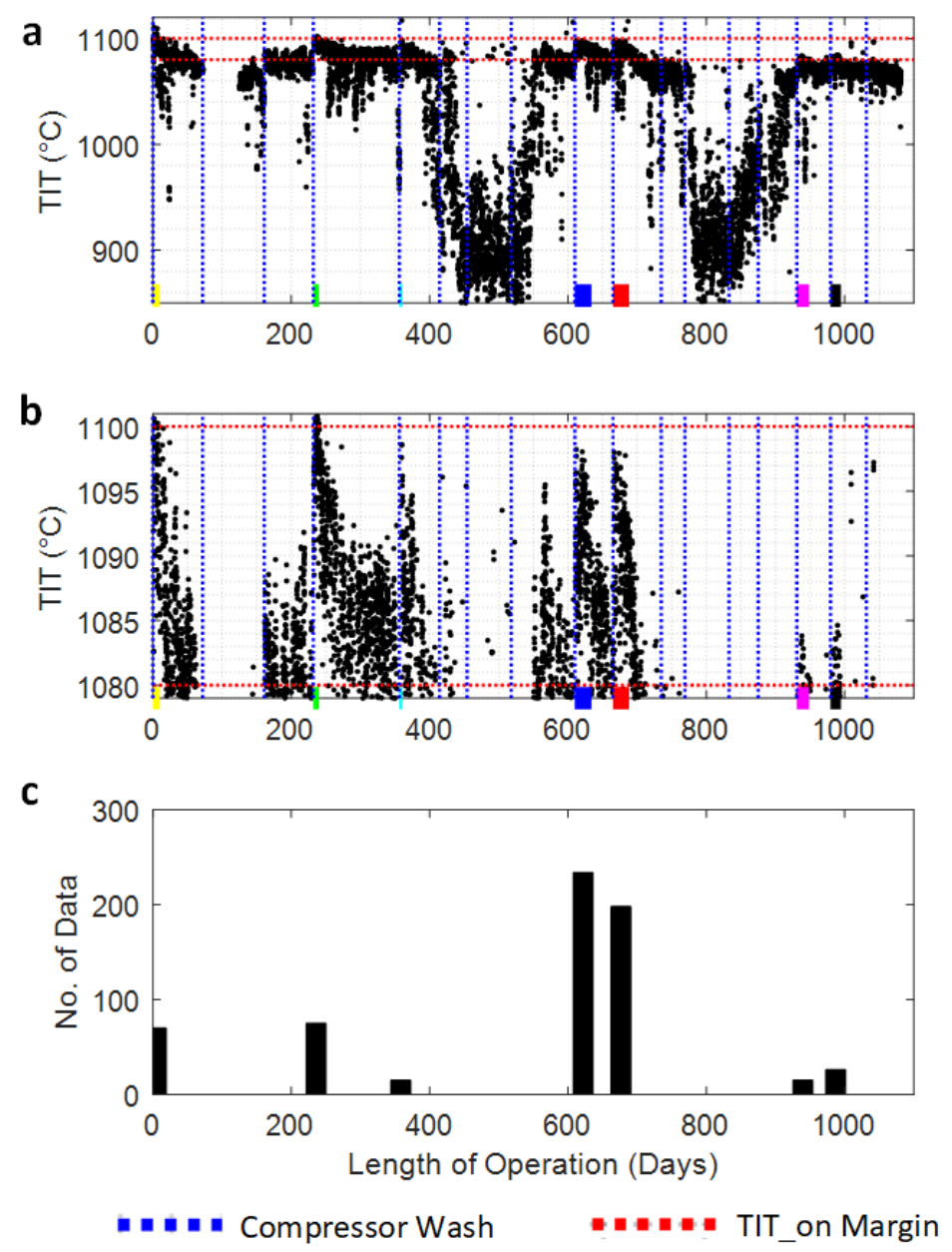

Figure 5-3: Epoch placement and data availability: variation of $T I T^{\prime}$ and placement of epoch for (a) global TIT range, (b) near TIT $_{o n}^{\prime}$, and (c) number of data points corresponding to TIT $_{o n}^{\prime}$ for each $\varepsilon_{i}$.

The epoch length, i.e., $\varepsilon_{T}$, was determined through examination of the $T I T_{D E V}$ indicator corresponding to each epoch. As seen in Figure 5-4, the $T I T_{D E V}$ variations of epoch $\varepsilon_{1}$ to $\varepsilon_{5}$ clearly indicated the fouling effect on the $T I T^{\prime \prime}$ evaluated by the turbinedegrading GPA model. The time right before the deviation in $T I T_{D E V}$ was used as the value of $\varepsilon_{T}$. The starting baseline value of $T I T_{D E V}$ for $\varepsilon_{6}$ and $\varepsilon_{7}$ were both 0.01 instead of zero, indicating the effect of the long-term turbine degradation on the $T I T^{\prime \prime}$. Physically, a GTE 
with a degraded turbine is expected to be operating at a lower TIT, due to the EGT limit imposed by the control system, as the turbine can no longer extract the same amount of energy for a given pressure ratio. Therefore, the lower $T I T^{\prime \prime}$ evaluated by the turbinedegrading GPA algorithm was valid and reflects the actual TIT. Since the TIT" of $\varepsilon_{6}$ and $\varepsilon_{7}$ were close to the lower bound of the sampling criteria, the entries with deviated $T I T^{\prime \prime}$ due to fouling effects were excluded from the sample. Contrary to the linearized fouling model proposed by Hanachi et al. [53], the observation of $T I T_{D E V}$ suggested a nonlinear fouling behaviour, which included a limited period where the fouling effect was not yet manifested as performance deterioration symptoms. This, however, is in accordance with the observations reported by Meher-Homji et al. [49]. 

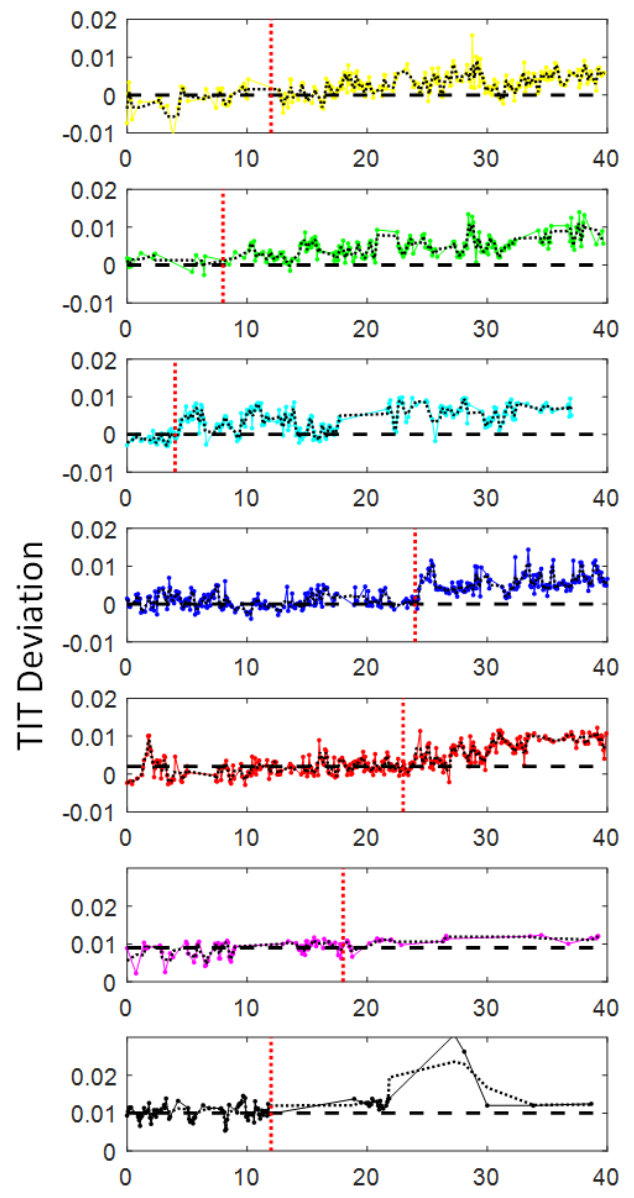

Days after each wash

..... Cut-off length of each epoch

.... Three-point moving mean

Figure 5-4: The $T_{I T} T_{D E V}$ variation indicated the effect on $T I T^{\prime \prime}$ by both the short-term fouling and the long-term turbine degradation.

\subsubsection{Establishment of the "Actual-Turbine" Performance Characteristics}

After establishing the epochs, the results from the turbine-degrading GPA were sampled and reconstructed into turbine performance characteristics, which are shown in Figure 5-5. The overall near-level slope of the constructed efficiency curves indicated that the sampled result entries were around the peak efficiency region of the curve. The colourcoded curves of different epochs revealed the gradual downward translation of the 
efficiency curve, as well as the modest elevation of the corrected mass flow over the operating history. The turbine performance curve transformation was consistent with the transformational signature of a turbine with a surface roughness fault, as suggested in Section 4.2.1. This revelation was backed by the early testing results of the Typhoon prototype, after which the examination of the turbine seal strips confirmed the absence of blade rubbing during GTE operation [54]. Combining the observations above, it was reasonable to exclude the tip-clearance enlargement from being a potential degradational fault and instead the turbine blade surface roughening was considered the predominant cause of the efficiency deviation.

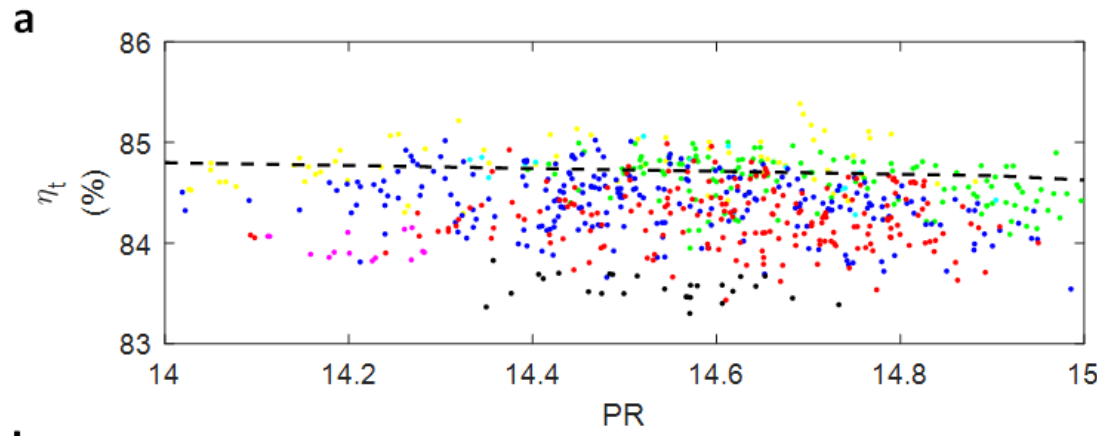

b

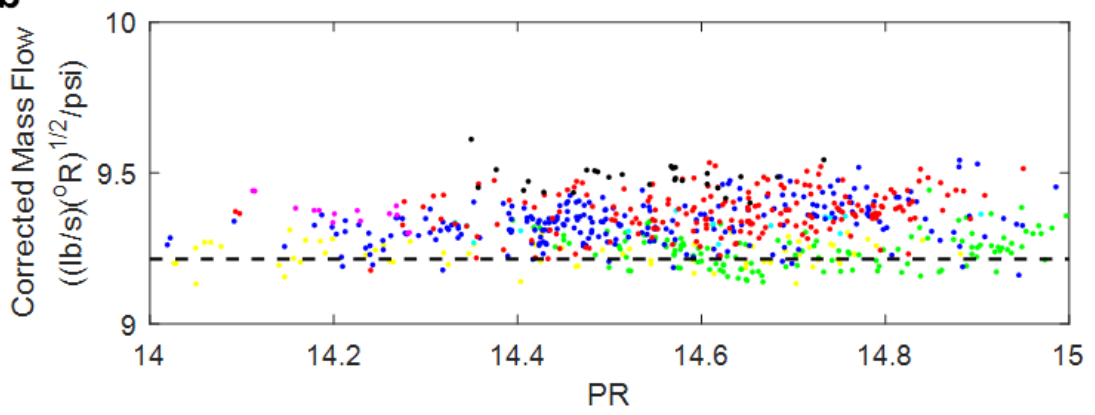

- - - B Baseline value from turbine performance characteristics map

Figure 5-5: Constructed turbine performance characteristics based on the results of the turbinedegrading GPA model: (a) downward efficiency curve shift and (b) slightly upward shift of the corrected mass flow. 
The turbine efficiency deviation, $\Delta \eta_{t}$, was calculated for every sample using the approximation outlined in Equation (4-1). Figure 5-6 (a) shows the frequency distribution of $\Delta \eta_{t}$. For all epochs other than $\varepsilon_{3}$, the distribution of $\Delta \eta_{t}$ appeared binomial, for which the median was considered representative. Due to small $\varepsilon_{T}$ values, the distribution of $\varepsilon_{3}$ was unclear and the mean value was taken as representative. Figure 5-6 (b) presents the established $\Delta \eta_{t}$ trend with the time of operation. From previous discussions on the transformation of turbine characteristics, as well as the reported observations during prototype testing, the $\Delta \eta_{t}$ growth trend was fully attributed to the surface roughness degradation of the present analysis. It was passed on to meanline severity identification without further processing.

a
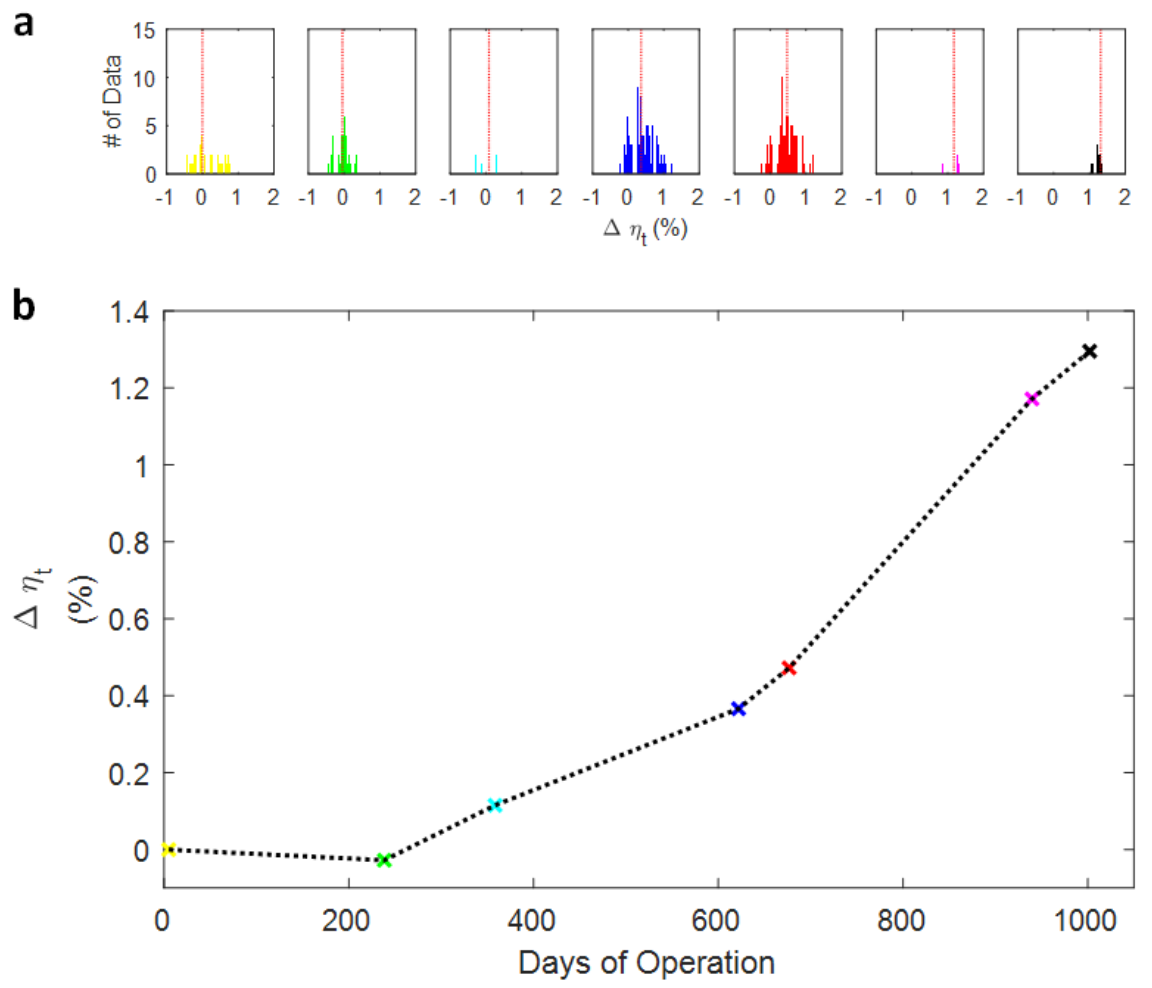

Figure 5-6: Formulation of the $\Delta \eta_{t}$ trend: (a) the frequency distribution of $\Delta \eta_{t}$ for each established epoch and (b) the final $\Delta \eta_{t}$ deviation trend with operating time. 
Figure 5-7 compares the efficiency loss "symptom," $\zeta_{\eta}$, obtained by the present epoch-based sampling method to the original result by Hanachi et al. [55], which was obtained using a fixed five-day sampling period after each wash without distinguishing between operating conditions. $\zeta_{\eta}$ was defined as the ratio of efficiency deviation to the actual efficiency, as in Equation (2-16). With an extended sample size and condition-based sampling criteria, $\zeta_{\eta}$ assessed by the present interfacing process showed a relatively modest and gradual growth trend compared to that by Hanachi et al. The difference was likely due to the following two reasons: (1) compared to sampling periods greater than 10 days, a five-day sampling period was still insufficient at gathering enough data to form a clear distribution of $\zeta_{\eta}$ and (2) without distinguishing between operating conditions, i.e., TIT, the distribution of $\zeta_{\eta}$ will be influenced by the result entries associated with a TIT significantly lower than TIT $_{\text {on }}$, resulting in differences in corrected rotor speed and lossto-roughness sensitivity.

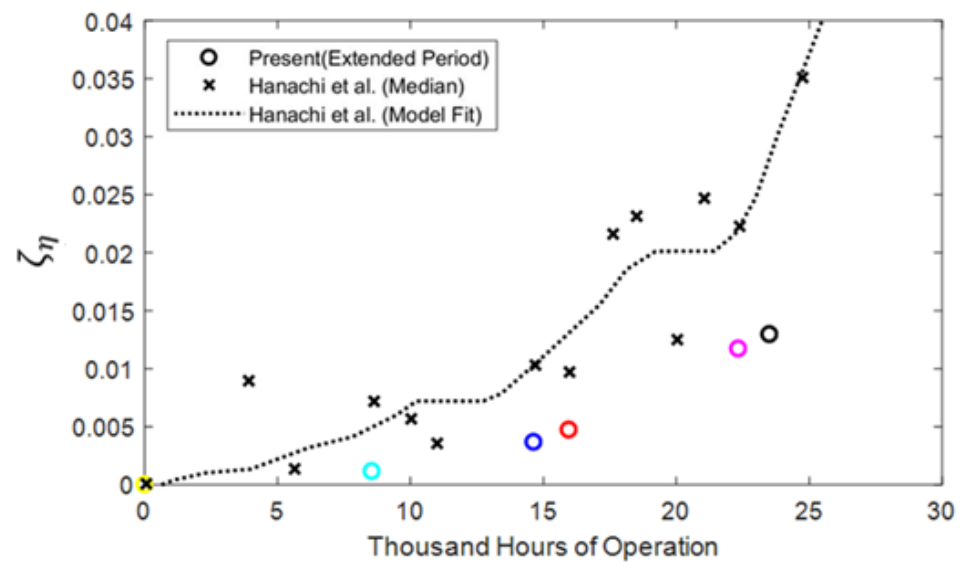

Figure 5-7: Comparison of the $\zeta_{\eta}$ trend obtained by the present interfacing process using the method of Hanachi et al. [55]. 


\subsection{Meanline Roughness Identification for the HP Turbine}

Using the design choices and rig-test performance outcomes summarized in Section 5.2, a meanline aerodynamic model was established for the HP turbine of the Typhoon GTE. Using the methodology presented in Section 2.3 and 4.3, the meanline representative velocity triangles were adjusted based on the streamline curvature throughflow analysis outcomes, such that the calculated performance characteristics matched the reported values in Table 5-2. Figure 5-8 shows the final velocity triangles for both the established meanline model and the original streamline curvature outcomes presented in [52]. The assumptions used to establish the meanline velocity triangles are also presented. Table ... summarizes the total pressure loss coefficients for various component losses outlined in Section 2.3.3. As seen in Table 5-5, apart from the flow coefficient, the calculated values were within 1\% agreement of the reported design and test rig values.
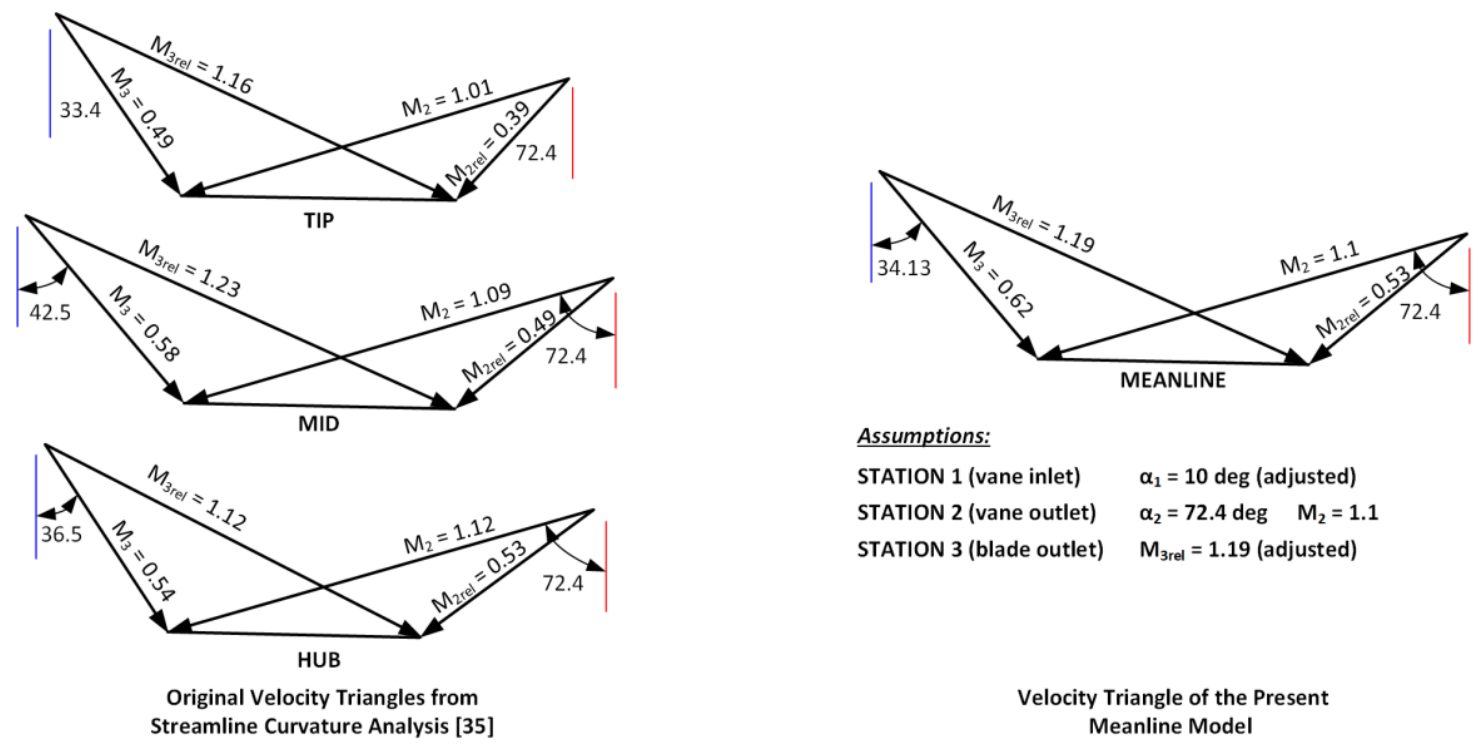

$$
\begin{array}{ll}
\text { STATION } 1 \text { (vane inlet) } & \alpha_{1}=10 \mathrm{deg} \text { (adjusted) } \\
\text { STATION } 2 \text { (vane outlet) } & \alpha_{2}=72.4 \mathrm{deg} \quad \mathrm{M}_{2}=1.1 \\
\text { STATION } 3 \text { (blade outlet) } & M_{3 \text { rel }}=1.19 \text { (adjusted) }
\end{array}
$$

Velocity Triangle of the Present Meanline Model

Figure 5-8: Established meanline velocity triangles compared with the streamline curvature analysis outcomes by Hannis et al. [52] for the design condition. 
Table 5-4: Assessed total pressure loss coefficients for various loss components.

\begin{tabular}{ccccccc}
\hline \multirow{5}{*}{ Vane } & & $\boldsymbol{Y}($ sum $)$ & $\boldsymbol{Y}_{\boldsymbol{P}}$ & $\boldsymbol{Y}_{\boldsymbol{S}}$ & $\boldsymbol{Y}_{\boldsymbol{T E T}}^{\prime}$ & $\boldsymbol{Y}_{\boldsymbol{T C}}$ \\
& Magnitude & 0.1946 & 0.0636 & 0.0961 & 0.0349 & - \\
& Percentage & $100 \%$ & $32.68 \%$ & $49.38 \%$ & $17.93 \%$ & - \\
\multirow{2}{*}{ Blade } & & & & & \\
& Magnitude & 0.3798 & 0.1595 & 0.0822 & 0.0341 & 0.1041 \\
& Percentage & $100 \%$ & $42 \%$ & $21.64 \%$ & $8.98 \%$ & $27.41 \%$ \\
\hline
\end{tabular}

Table 5-5: Comparison of performance parameters between the established meanline model and the reported reference values from Hannis et al. [52].

\begin{tabular}{ccc}
\hline $\begin{array}{c}\text { Performance } \\
\text { Parameter }\end{array}$ & $\begin{array}{c}\text { Design/ } \\
\text { Rig-Testing [52] }\end{array}$ & Meanline Model \\
$\eta_{t_{\text {stage }}}$ & $85.5 \%$ & $84.8 \%$ \\
$P R_{\text {stage }}$ & 3.9 & 3.92 \\
$\psi_{\text {stage }}$ & 2.0 & 2.01 \\
$\phi_{\text {stage }}$ & 0.57 & 0.52 \\
$\Lambda_{\text {stage }}$ & 0.45 & 0.44 \\
\hline
\end{tabular}

Based on the established meanline model of the HP turbine, the surface roughness loss damage model presented in Section 2.4.2 was incorporated. To make the surface roughness comparable to measured values from Tarada [7], which were presented in terms of centre-line average $(R a)$, the following correlation from Koch \& Smith [56] was applied to the sand-grain roughness height:

$$
R a \cong \frac{k_{s}}{6.2}
$$

Figure 5-9 shows the isentropic efficiency loss trend with the "Quarter Surface Coverage Index" defined in Section 4.3.1 in terms of $R a$ for the HP turbine. At first glance, the efficiency drop may appear too optimistic compared to the efficiency drop reported in 
the literature, such as the $7 \%$ drop for $k_{s} / C$ of 0.01 by Bammert $\&$ Sandstede [38] and the $19 \%$ drop for $k_{s} / C$ of 0.0067 by Yun et al. [40]. However, the Mach number ranges corresponding to these two reported cases were extremely low, with nearly no compressibility effects, which have a large impact on the roughness incremental loss, as previously shown in Figure 3-3.

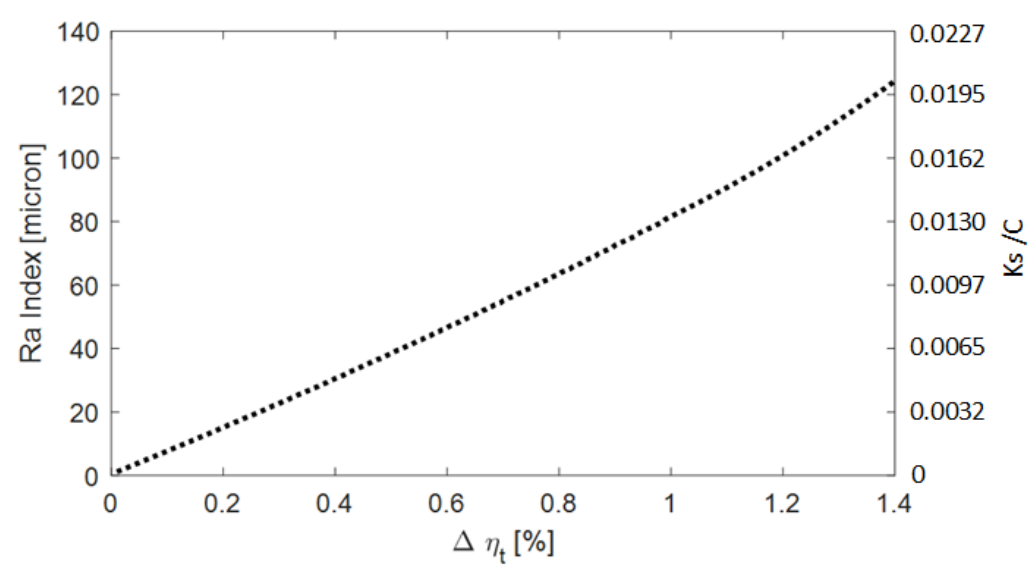

Figure 5-9: Variation of the "Quarter Surface Coverage Index" with the isentropic efficiency loss.

Finally, the growth trend of the surface roughness in terms of the "Quarter Surface Coverage Index" is presented in Figure 5-10. Blade samples were unavailable from the operator, so the assessed trend and magnitude of the index were compared with the measurements by Tarada [7], which included the operating history associated with each blade sample. Fortunately, among the measured data, there was a handful of IN939 blade samples from the HP turbine stages of the engines by Ruston, the original designer and manufacturer of the Typhoon GTE. Thus, the data associated with Ruston blade samples are highlighted. 

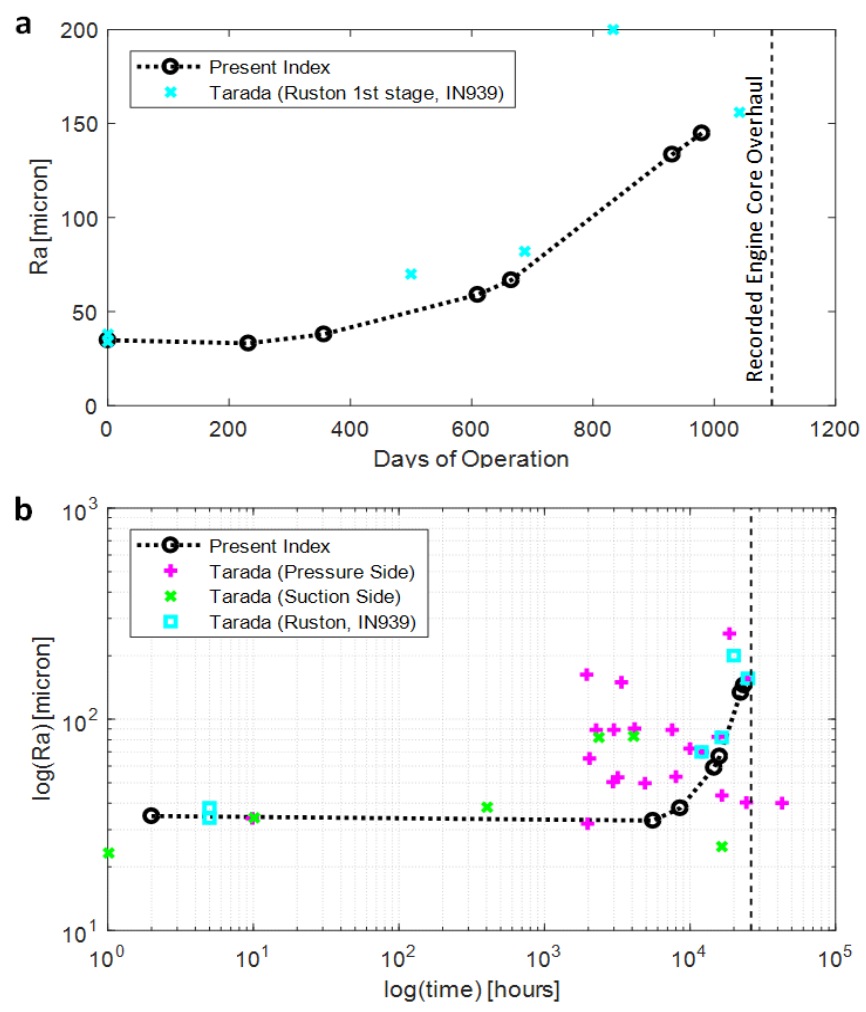

Figure 5-10: Growth trend of surface roughness in terms of the "Quarter Surface Coverage Index": (a) comparison with the measurements on the IN939 blade samples from Ruston HP turbines, (b) comparison with all available measurements by Tarada [7].

To make the present results comparable with other IN939 measurements, an initial centre-line average surface roughness of 40 microns was assumed. Figure 5-10 (a) shows the surface roughness index, in both trend and magnitude agreement with the measured values from the Ruston IN939 samples. Figure 5-10 (b) compares the assessed surface roughness index with all available measurements by Tarada. The comparison shows that the surface roughness index of the monitored turbine reached the peak magnitude level shown in the measured data as the operating time of the GTE approached the recorded engine-core overhaul. 


\section{Chapter 6: Conclusions and Recommendations}

\subsection{Conclusions}

In this work, a physics-based online assessment framework was developed to assess the surface roughness level of the HP turbine in an industrial GTE. It integrated the advantages of both GPA and meanline turbine modelling.

Since the meanline modelling component of the framework utilized the Islam \& Sjolander empirical roughness loss correlations [12] as well as the compressibility correction factor for roughness loss proposed by Jouini et al. [10], the validity of the correlations was first evaluated. The validation was based on the recent transonic roughness loss measurements by Yuan [41] using the HS1C transonic cascade. Yuan's data confirmed the CFD-based observations by Jouini et al., that is, the incremental loss due to roughness diminishes as the Mach number is increased. A modification was made to the compressibility correction factor based on Yuan's data, which resulted in improved roughness loss prediction. Despite the deficiencies in the prediction of the baseline profile losses by the AMDCKO loss system, the predicted roughness incremental losses showed acceptable agreement with the measured results.

The components of the proposed online framework were then presented. In the GPA-meanline interfacing process, an epoch-based sampling method, as well as the TIT deviation indicator $\left(T I T_{D E V}\right)$ were proposed to alleviate the uncertainties associated with the GPA outcomes, based on basic GTE instrumentation. A process was proposed for the development of the meanline-based surface roughness index for a specific turbine design. 
The effectiveness of the proposed online-assessment framework was demonstrated by an application to industrial GTE operational data over a three-year period between two major overhauls. The proposed $T I T_{D E V}$ indicator clearly revealed the nonlinear behaviour of the fouling influence on the GPA results and was effectively used to maximize the acquirable sample size for each established epoch. With increased sample size and condition-specific sampling criteria, the interfacing process was able to alleviate the uncertainties associated with scantiness of data and different operating conditions. This, in turn, yielded a different observation on the performance deterioration symptom compared to the value reported by Hanachi et al. [55] on the same set of data. Finally, the growth trend of the proof-of-concept "Quarter Surface Coverage Index" was determined for the entire three-year operating period. The index showed both trend and magnitude agreement with the available measured values of comparable service hours reported by Tarada [7], demonstrating the validity of the proposed surface roughness assessment approach as well as its potential as an effective online monitoring tool.

\subsection{Recommendations}

The present surface-roughness indicator only serves as a low-fidelity, proof-ofconcept assessment, as it was developed based on the exhaustive utilization of both logged operational data and literature. The indicator attributes all roughness damage loss to $25 \%$ of the surface area on the suction side near the leading edge. While such an indicator definition can provide some physical insight on the roughness condition of the turbine blade, it does not reflect the variation of roughness severity on the blade surface, especially for the pressure side where the in-service roughness damage is reportedly more severe [6]. 
A more advanced definition of an indicator is required to further quantify the surface roughness level at various locations on the airfoil, especially for associating the surface roughness indication with component failure.

The meanline modelling approach may be replaced by the 2D throughflow analysis. In general, 2D throughflow analysis is a better reverse-engineering tool compared to meanline modelling by accounting the radial pressure gradients and the span-wise variation of flow conditions. From the perspective of damage modelling, the 2D analysis also allows damage effects to be concentrated at a specific span location, as opposed to the present approach where the damage effects are averaged across the span. Such a 2D-based "concentrated" damage modelling approach has been successfully demonstrated by Klein in simulating the tip-clearance damage effects, which resulted in more realistic predictions of the spanwise temperature distributions and the overall efficiency penalties caused by the damage [57]. Klein's work shows promise in incorporating such an approach of damage effects predictions into the framework.

In the discussion on the GPA-meanline interfacing process, a fault feature extraction concept based on the turbine performance characteristic transformation was briefly mentioned. Although realization of the performance characteristic transformation by different fault types was made almost three decades ago by Isaacs [24], no specific publications exist in the public domain to establish such a link. A future investigation might focus on developing such a correlation, which allows for the expansion of the framework to the assessment of other fault types, including trailing edge damage and tip-clearance enlargement. 


\section{References}

[1] Y. G. Li, "Performance-analysis-based gas turbine diagnostics: A review," Proc. Inst. Mech. Eng. Part A J. Power Energy, vol. 216, no. 5, pp. 363-377, Jan. 2002.

[2] I. S. Diakunchak, "Performance Deterioration in Industrial Gas Turbines," ASME. J. Eng. Gas Turbines Power, vol. 114, no. 2, p. 161, 1992.

[3] J. P. Bons, R. P. Taylor, S. T. McClain, and R. B. Rivir, "The Many Faces of Turbine Surface Roughness,” ASME. J. Turbomach., vol. 123, no. 4, p. 739, 2001.

[4] J. P. Bons, "A Review of Surface Roughness Effects in Gas Turbines," ASME. J. Turbomach., vol. 132, no. 2, p. 021004, 2010.

[5] A. L. Chandler and A. R. Finkelstein, "Turbine Blade Tip Clearance Measurement Utilizing Borescope Photography," in ASME. Turbo Expo: Power for Land, Sea, and Air, Volume 1B: General. V01BT02A063. doi:10.1115/78-GT-164.

[6] R. P. Taylor, "Surface Roughness Measurements on Gas Turbine Blades," ASME. J. Turbomach., vol. 112, no.2, p. 175, 1990.

[7] F. Tarada, "Heat Transfer to Rough Turbine Blading," Ph.D. Thesis, Universty of Sussex, 1987.

[8] R. J. Kind, P. J. Serjak, and M. W. P. Abbott, "Measurements and Prediction of the Effects of Surface Roughness on Profile Losses and Deviation in a Turbine Cascade,” ASME. J. Turbomach., vol. 120, no. 1, p. 20, 1998.

[9] L. Q. Yuan and R. J. Kind, "Measurements and Computations of Compressible Flow Through a Turbine Cascade with Surface Roughness," in ASME Turbo Expo: Power for Land, Sea and Air, Volume 6: Turbomachinery, Parts A and B. pp. 1-9. 
doi:10.1115/GT2006-90018.

[10] D. B. Jouini, A. M. T. Islam, and S. A. Sjolander, "Effect of surface roughness on profile losses in axial turbines at transonic mach numbers," Can. Aeronaut. Sp. J., vol. 44, no. 4, pp. 263-272, 1998.

[11] H. Hanachi, "Gas Turbine Engine Performance Estimation and Prediction," Ph.D. Thesis, Carleton University, 2015.

[12] A. M. T. Islam, "An experiemental and computational study of the aerodynamics of turbine blades with damage," Ph.D. Thesis, Carleton University, 1999.

[13] S. De Cecco, M. I. Yaras, and S. A. Sjolander, "Measurements of the Tip-Leakage Flow in a Turbine Cascade with Large Clearances," ASME 1995 Int. Gas Turbine Aeroengine Congr. Expo., 1995.

[14] H. Hanachi, J. Liu, A. Banerjee, and Y. Chen, "A framework with nonlinear system model and nonparametric noise for gas turbine degradation state estimation," Meas. Sci. Technol., vol. 26, no. 6, p. 65604, 2015.

[15] E. S. Hendricks, "Meanline Analysis of Turbines with Choked Flow in the ObjectOriented Turbomachinery Analysis Code," in AIAA SciTech, 2016.

[16] C. Maclean, "Effects of Real Fluid Properties on Axial Turbine Meanline Design and Off-Design,” M.A.Sc. Thesis, Carleton University, 2012.

[17] H. I. H. Saravanamuttoo, C. F. Rogers, H. Cohen, and P. V. Straznicky, Gas turbine theory. Pearson Prentice Hall, 2009.

[18] J. D. Denton, “Loss Mechanisms in Turbomachines," ASME. J. Turbomach., vol. 115, no. 4, p. 621, 1993. 
[19] S. C. Kacker and U. Okapuu, “A Mean Line Prediction Method for Axial Flow Turbine Efficiency,”ASME. J. Eng. Power, vol. 104, no. 1, p. 111, 1982.

[20] D. G. Ainley and C. R. Mathieson, “A Method of Performance Estimation for AxialFlow Turbines," Aeronaut. Res. Counc. reports Memo. N. 2974, 1951.

[21] H. R. M. Craig and H. J. A. Cox, "Performance Estimation of Axial Flow Turbines," Proc. Inst. Mech. Eng., 1970.

[22] O. E. Baljé and R. L. Binsley, “Axial Turbine Performance Evaluation,” ASME. J. Eng. Gas Turbines Power, 1968.

[23] J. Zhu and S. A. Sjolander, "Improved Profile Loss and Deviation Correlations for Axial-Turbine Blade Rows," in ASME. Turbo Expo: Power for Land, Sea, and Air, Volume 6: Turbo Expo 2005, Parts $A$ and $B$, 2005, pp. 783-792. doi:10.1115/GT2005-69077.

[24] D. P. Isaacs, "The aerodynamics of turbine blades with trailing edge damage.," M.Eng Thesis, Carleton University, 1994.

[25] M. W. Benner, "The influence of leading-edge geometry on profile and secondary losses in turbine cascades," Ph.D. Thesis, Carleton University, 2003.

[26] M. I. Yaras and S. A. Sjolander, "Prediction of Tip-Leakage Losses in Axial Turbines,” ASME. J. Turbomach., vol. 114, no. 1, p. 204, Jan. 1992.

[27] R. H. Aungier, Turbine Aerodynamics: Axial-Flow and Radial-Inflow Turbine Design and Analysis, 1st ed. New York: ASME, 2005.

[28] M. Cicciotti, D. P. Xenos, A. E. F. Bouaswaig, N. F. Thornhill, and R. F. MartinezBotas, “Online Performance Monitoring of Industrial Compressor Using Meanline 
Modelling," in ASME TurboExpo 2014: Turbine Technical Conference and Exposition, V02DT42A003. doi:10.1115/GT2014-25088.

[29] S. H. Moustapha, S. C. Kacker, and B. Tremblay, “An Improved Incidence Losses Prediction Method for Turbine Airfoils," ASME. J. Turbomach., vol. 112, no. 2, p. 267, Apr. 1990.

[30] P. R. Bandyopadhyay, "Rough-wall turbulent boundary layers in the transition regime," J. Fluid Mech., vol. 180, no. 1, p. 231, Jul. 1987.

[31] T. Cebeci and K. C. Chang, "Calculation of incompressible rough-wall boundarylayer flows," AIAA J., vol. 16, no. 7, pp. 730-735, 1978.

[32] D. Jouini, T. Bergenblock, and S. Sjolander, "Computation of the Aerodynamic Performance of Axial-Turbine Blades with Surface Roughness," in International Symposium on Air Breathing Engines, 1997, pp. 250-259.

[33] F. A. Dvorak, "Calculation of turbulent boundary layers on rough surfaces in pressure gradient.," AIAA J., vol. 7, no. 9, pp. 1752-1759, Sep. 1969.

[34] R. L. Simpson, "A generalized correlation of roughness density effects on the turbulent boundary layer.," AIAA J., vol. 11, no. 2, pp. 242-244, Feb. 1973.

[35] A. Sigal and J. E. Danberg, "New correlation of roughness density effect on the turbulent boundary layer," AIAA J., vol. 28, no. 3, pp. 554-556, Mar. 1990.

[36] J. Nikuradse, “Laws of Flow in Rough Pipes,” NACA TM 1292, Washington, 1950.

[37] H. Schlichting, Boundary-Layer Theory. McGraw-Hill, 1979.

[38] K. Bammert and H. Sandstede, "Measudements of the Boundary Layer Development along a Turbine Blade with Rough Surfaces," ASME. J. Eng. Power, 
vol. 102, no. 4, pp. 978-983, 1980.

[39] J. L. Boynton, R. Tabibzadeh, and S. T. Hudson, "Investigation of Rotor Blade Roughness Effects on Turbine Performance,”ASME. J. Turbomach., vol. 115, no. 3, p. 614, Jul. 1993.

[40] Y. Il Yun, I. Y. Park, and S. J. Song, "Performance Degradation due to Blade Surface Roughness in a Single-Stage Axial Turbine," ASME. J. Turbomach., vol. 127, no. 1, p. $137,2005$.

[41] L. Q. Yuan, "Measurements and Computatilns of Roughness Effects on Performance of a HP Turbine Cascade in Compressible Flow.” M.A.Sc. Thesis, Carleton University, 2004.

[42] D. Corriveau, "Influence of Loading Distribution on the Performance of High Pressure Turbine Blades,” Ph.D. Thesis, Carleton University, 2005.

[43] D. Corriveau and S. A. Sjolander, "Influence of Loading Distribution on the Performance of Transonic High Pressure Turbine Blades," ASME. J. Turbomach., vol. 126, no. 2, p. 288, Apr. 2004.

[44] M. S. Jeffries, "Initial investigations of transonic turbine aerodynamics using the Carleton University high-speed wind tunnel," Ph.D. Thesis, Carleton University, 2000.

[45] C. Sooriyakumaran, "Experimental Study Of Profile Losses in Three Transonic Turbine Cascades,” M.A.Sc. Thesis, Carleton University, 2014.

[46] M. D. Kibsey and S. A. Sjolander, "Influence of Mach Number on Profile Loss of Axial-Flow Gas Turbine Blades," in ASME. Turbo Expo: Power for Land, Sea, and 
Air, Volume 2B: Turbomachinery, 2016, p. V02BT38A015. doi:10.1115/GT201656410.

[47] B. MacIsaac and R. Langton, Gas Turbine Propulsion Systems, 1st ed. Chichester, West Sussex, UK: Wiley, 2011.

[48] R. J. Boyle, "Prediction of Surface Roughness and Incidence Effects on Turbine Performance,” ASME. J. Turbomach., vol. 116, no. October, pp. 745-751, 1994.

[49] C. Meher-Homji and A. Bromley, "Gas Turbine Axial Compressor Fouling and Washing," Turbomach. Symp., pp. 163-191, 2004.

[50] F. Carchedi, A. M. Campling, and G. R. Wood, "Design and Development of the Ruston Typhoon Gas Turbine,” Turbomach. Int., vol. 00, no. c, pp. 906-916, 1989.

[51] A. T. Sanders, S. Gallimore, and P. Whitehouse, "A Power Increase of 17\% for the EGT Typhoon Gas Turbine," in ASME. Turbo Expo: Power for Land, Sea, and Air, Volume 3C: General, 1993, p. V03CT17A092. doi:10.1115/93-GT-431.

[52] J. M. Hannis, R. Maali, and G. Woollatt, "The design and development of a high work transonic turbine stage," in Proceeding of the Institution of Mechanical Engineer. Turbomachinery: Latest Developments in a Changing Scene, 1991, pp. 33-45.

[53] H. Hanachi, J. Liu, A. Banerjee, Y. Chen, and A. Koul, “A Physics-Based Modeling Approach for Performance Monitoring in Gas Turbine Engines," IEEE Trans. Reliab., vol. 64, no. 1, pp. 197-205, 2015.

[54] F. Carchedi, S. Gallimore, A. F. Siemiernik, and D. A. Goodman, "Development and Early Field Experience of the Typhoon Gas Turbine," in International Gas 
Turbine \& Aeroengine Congress and Exposition, 1991.

[55] H. Hanachi, J. Liu, A. Banerjee, and Y. Chen, "Sequential state estimation of nonlinear/non-Gaussian systems with stochastic input for turbine degradation estimation,” Mech. Syst. Signal Process., vol. 72-73, pp. 32-45, 2016.

[56] C. C. Koch and L. H. Smith, "Loss Sources and Magnitudes in Axial-Flow Compressors,” ASME. J. Eng. Power, vol. 98, no. 3, p. 411, Jul. 1976.

[57] W. A. Klein, "Contributions to the study of the aerodynamics of damaged turbine blades.," M.A.Sc. Thesis, Carleton University, 1993. 


\section{Appendix A: HS1C Cascade Performance Data by Corriveau and Yuan}

Data from Corriveau:

Table A-1: HS1C Cascade Performance Data at Design Incidence, adapted from Ref. [42].

\begin{tabular}{||c|c|c|c|c|c|c|c||}
\hline$M_{2 \text { Mo }}$ & $M_{1}$ & $\operatorname{Re}_{2}\left(\times 10^{-3}\right)$ & AVDR & $\xi$ & $\alpha 2$ & $Y_{t}$ & $Y_{\text {pwe }}$ \\
\hline \hline 0.534 & 0.394 & 477 & 1.02 & 0.050 & 55.6 & 0.064 & 0.060 \\
\hline 0.729 & 0.495 & 652 & 1.00 & 0.038 & 56.3 & 0.057 & 0.050 \\
\hline 0.879 & 0.531 & 776 & 0.99 & 0.033 & 57.0 & 0.057 & 0.047 \\
\hline 1.003 & 0.545 & 868 & 0.98 & 0.040 & 57.2 & 0.079 & 0.062 \\
\hline 1.103 & 0.545 & 952 & 0.98 & 0.037 & 56.7 & 0.082 & 0.062 \\
\hline 1.195 & 0.549 & 995 & 0.96 & 0.055 & 55.8 & 0.138 & 0.100 \\
\hline 1.296 & 0.548 & 1039 & 0.97 & 0.063 & 54.1 & 0.181 & 0.125 \\
\hline
\end{tabular}

Roughness loss data from Yuan:

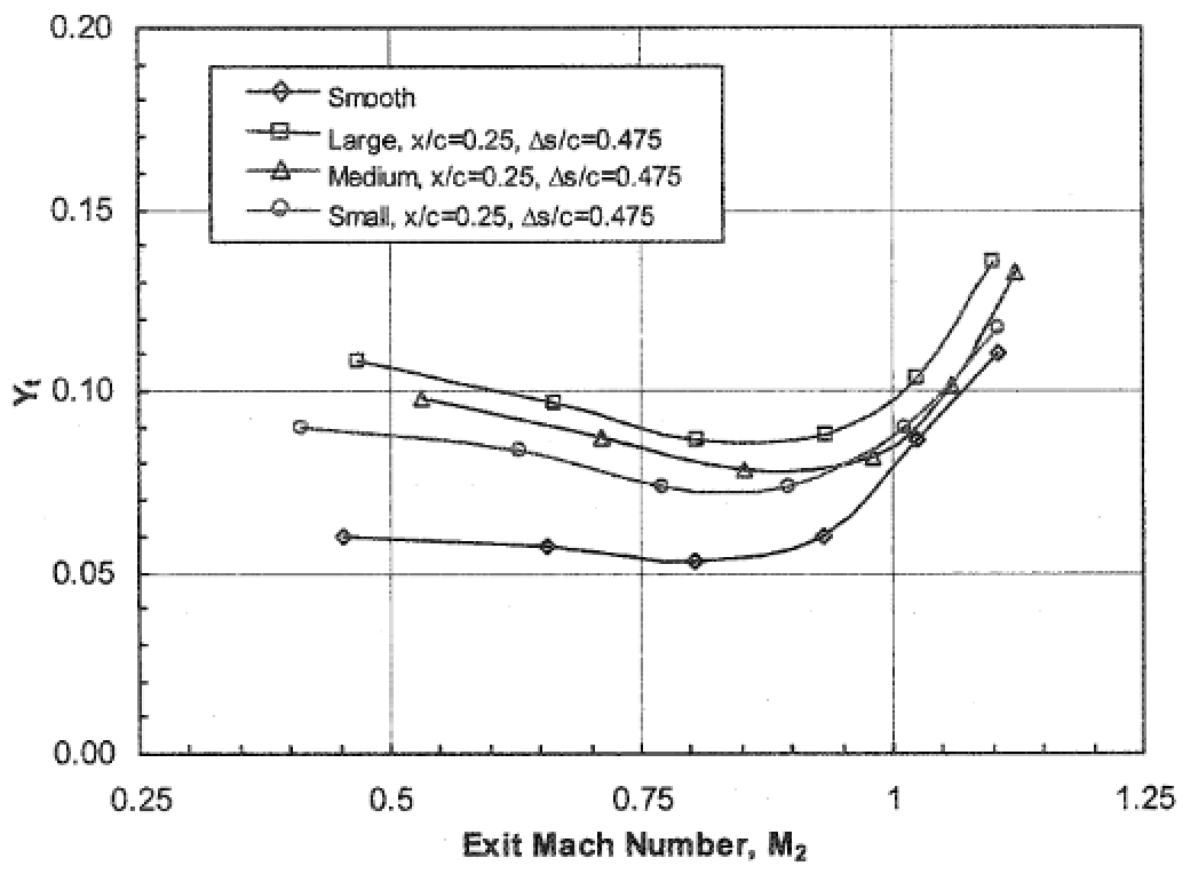

Figure A-1: Variation of total pressure loss coefficient with exit Mach number for roughness configuration of $x / C=0.25, \Delta s / C=0.475$ with various roughness heights, adapted from Ref. [41]. 


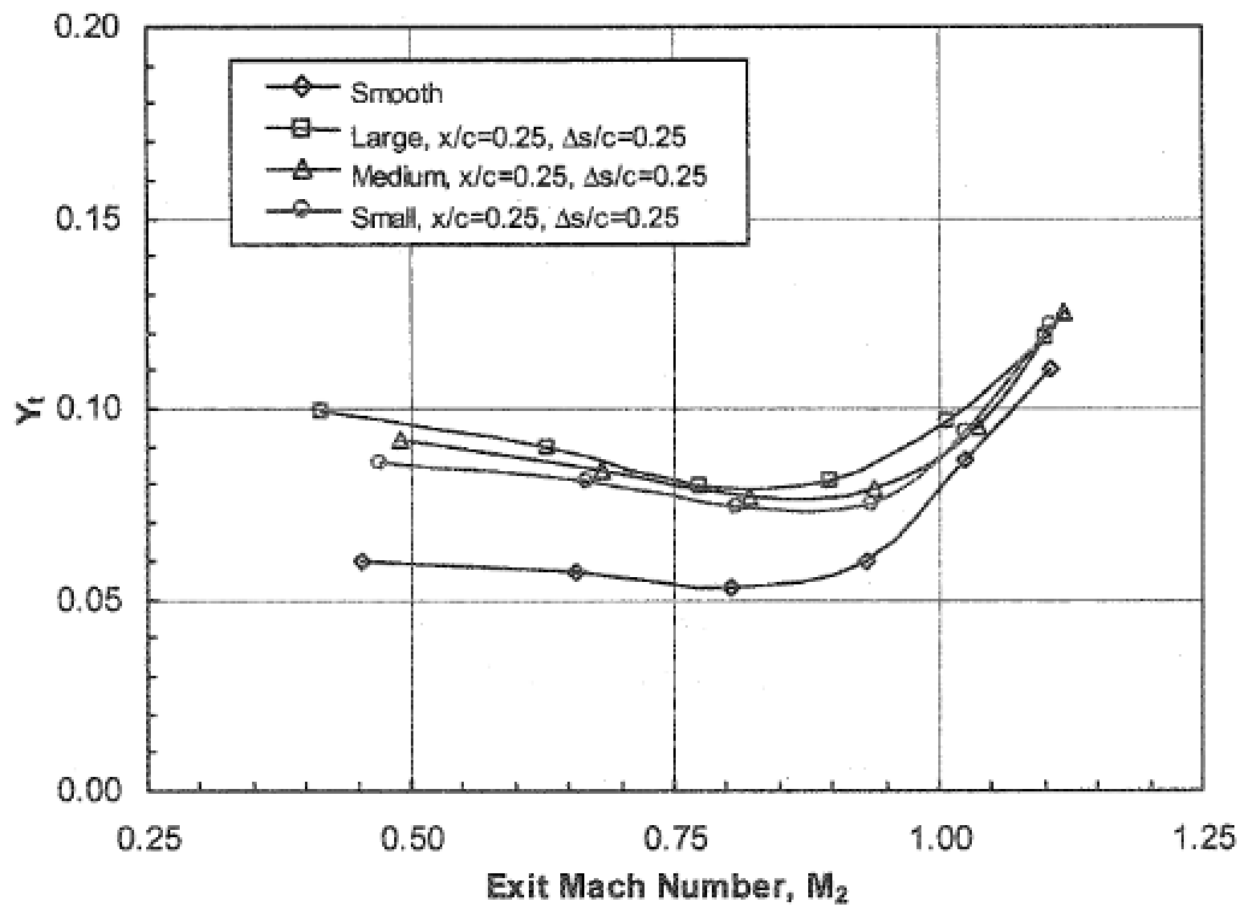

Figure A-2: Variation of total pressure loss coefficient with exit Mach number for roughness configuration of $x / C=0.25, \Delta s / C=0.25$ with various roughness heights, adapted from Ref. [41]. 